\title{
Symplectic and Poisson derived geometry and deformation quantization
}

\author{
T. Pantev, G. Vezzosi
}

\begin{abstract}
We review recent results and ongoing investigation of the symplectic and Poisson geometry of derived moduli spaces, and describe applications to deformation quantization of such spaces.
\end{abstract}

\section{Contents}

Introduction 1

1 Shifted symplectic structures 5

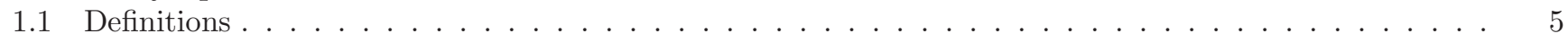

1.2 Existence theorems and examples . . . . . . . . . . . . . . . . . . . . . 8

2 Shifted Poisson structures 13

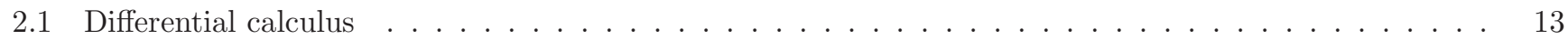

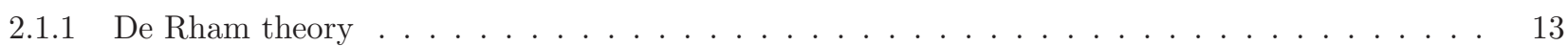

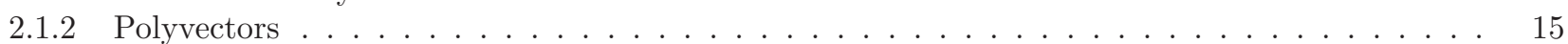

2.1 .3 Standard realizations over $k \ldots \ldots \ldots$

2.1.4 Comparison between Poisson algebras and shifted Poisson pairs . . . . . . . . . . . . . . . 17

2.1.5 From non-degenerate Poisson algebra structures to symplectic structures . . . . . . . . . . . . 19

2.1 .6 Tate realizations over $k$. . . . . . . . . . . . . . . . . . . . . . . 20

2.2 Formal derived stacks and formal localization . . . . . . . . . . . . . . . . . . . . . 22

2.2.1 Formal derived stacks . . . . . . . . . . . . . . . . . . . . . . . . . 22

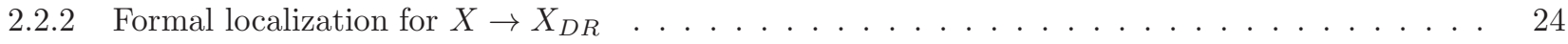

2.3 Shifted Poisson structures . . . . . . . . . . . . . . . . . . . . . . . . . . 27

2.4 Comparison between non degenerate shifted Poisson structures and shifted symplectic structures . . . 28

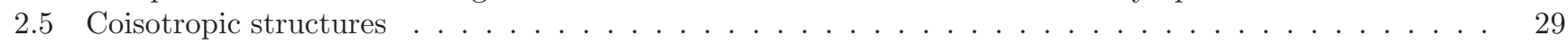

3 Deformation quantization $\quad 30$

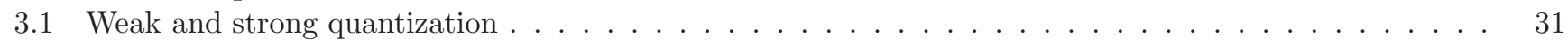

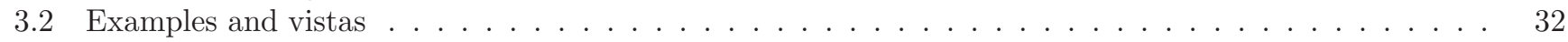

\section{Introduction}

From the vantage point of the timeline of the AMS Summer Institutes, this contribution is a continuation and an update of B. Toën's 2005 overview [To1]. Our goal here is to highlight some of the remarkable developments in derived geometry that we witnessed in the past ten years.

Our main topics - symplectic and Poisson geometry on derived moduli spaces - are among the latest topics in the area. Even though the study of these topics is still in an early stage, it has already led to some exciting applications. Among the moduli theoretic applications, we might mention the fact that $(-1)$-shifted symplectic structures induce symmetric perfect obstruction theories in the sense of [Be-Fa] (and actually all the geometrically interesting examples of symmetric obstruction theories arise this way), and the related important result that the Donaldson-Thomas moduli space is $(-1)$-shifted symplectic and Zariski locally isomorphic to the critical locus of a potential [BBJ.

Section 1 explains and summarizes the main results from [PTVV], while sections 2 and 3 delve into the substance of [CPTVV]. The aim of our review has been twofold: on one hand to convey the intuition behind definitions, 
constructions, and proofs of the main results, and on the other hand, to explain and motivate the slight change of point of view going from [PTVV] to [PTVV]. The upshot is that shifted Poisson geometry and its applications to deformation quantization of derived categories require a new broader perspective and new technical tools, i.e. differential calculus in an extremely general setting (Section 2.1), and formal localization (Section 2.2). Even though these powerful tools were created in order to solve our specific problems related to shifted Poisson structures, they constitute also a conceptual advance, very likely to become relevant in other contexts and to different problems in derived algebraic geometry.

Note that there is a parallel theory of shifted quadratic forms on derived moduli spaces, but we will not review it here (see Vez2 for a first investigation).

We now describe, section by section, the mathematical contents of this paper in more details.

Shifted symplectic structures. A shifted symplectic structure on a derived stack $X$ with a perfect cotangent complex $\mathbb{L}_{X}$ is a structured self-duality of $\mathbb{L}_{X}$ up to a shift, i.e. a quasi-isomorphism $\mathbb{T}_{X} \simeq \mathbb{L}_{X}[n]$ induced by a closed $n$-shifted 2 -from on $X$. The idea is an obvious generalization of the classical definition of symplectic form, but with an important additional, purely derived algebro-geometrical feature: for a shifted form on $X$, being closed is not a property but rather a datum. In other words, there is a canonical map, called the underlying-form map, from the space $\mathcal{A}^{p, \mathrm{cl}}(X, n)$ of $n$-shifted closed $p$-forms on $X$, to the space $\mathcal{A}^{p}(X, n)$ of $n$-shifted $p$-forms on $X$, but this map is not, in general, "injective" in any reasonable sense of the word (e.g. not injective on the connected components of these spaces). The space $\operatorname{Sympl}(X, n)$ is exactly the subspace of $\mathcal{A}^{2, c l}(X, n)$ of closed 2-forms whose underlying 2 -from is non-degenerate, i.e. such that the induced map $\mathbb{T}_{X} \rightarrow \mathbb{L}_{X}[n]$ is a quasi-isomorphism. Shifted symplectic structures abound, in the sense that many moduli spaces of interest to algebraic geometers and topologists, such as the moduli spaces of principal bundles or perfect complexes on algebraic Calabi-Yau manifolds or compact orientable topological manifolds, have derived enhancements carrying natural shifted symplectic structures. In Section 1 we give three general existence results for shifted symplectic structures on derived moduli stacks, leading to a long list of examples.

Shifted Poisson structures. Having at our disposal a theory of shifted symplectic structures, it is natural to look for a more general theory of shifted Poisson structures on derived moduli stacks. Actually, our original motivation for such a general theory came from the expected link between a shifted Poisson structure on a derived stack and an induced deformation quantization of its dg-derived category of perfect complexes. We will say more about this motivationapplication below. While classically, setting up a theory of Poisson varieties does not present more difficulties than setting up a theory of symplectic varieties, in derived algebraic geometry the situation is radically different. The usual bad functoriality properties of shifted polyvectors (as opposed to the good functoriality of closed shifted forms) together with the very delicate and intricate strictification problems related to establishing a meaningful shifted Poisson algebra structure on them, immediately made us realize that, outside the derived Deligne-Mumford case, a full-fledged theory of shifted Poisson structures on derived Artin stacks required new ideas and tools. If $X$ is a derived Artin stack, locally of finite presentation over the base $k$ (always assumed to be a Noetherian commutative $\mathbb{Q}$-algebra), then its cotangent complex $\mathbb{L}_{X}$ is perfect, and we may consider the graded commutative differential graded algebra

$$
\operatorname{Pol}(X, m):=\bigoplus_{p \geq 0} \Gamma\left(X, \operatorname{Sym}^{p}\left(\mathbb{T}_{X}[-m]\right)\right.
$$

of $m$-shifted polyvectors on $X$. Here, $m \in \mathbb{Z}, \mathbb{T}_{X}$ is the tangent complex of $X$, i.e. the $\mathcal{O}_{X}$-dual of $\mathbb{L}_{X}, \Gamma$ denotes the derived functor of global sections (i.e. hypercohomology), and the external grading, called weight grading, is given by $p$. In order to define shifted Poisson structures on $X$, we have to endow $\operatorname{Pol}(X, m)$ with a degree $m$ and weight -1 Lie bracket, making it into a graded $\mathbb{P}_{m+1}$-commutative differential graded algebra over $k$. In particular, $\operatorname{Pol}(X, m)[m]$ will be a graded dg-Lie algebra over $k$, with a weight -1 Lie bracket. Then we could adopt the following derived variation of a classical definition of Poisson structure, and put

$$
\operatorname{Poiss}(X, n):=\operatorname{Map}_{\mathrm{dglie}_{k}^{g r}}(k(2)[-1], \operatorname{Pol}(X, n+1)[n+1])
$$

for the space Poiss $(X, n)$ of $n$-shifted Poisson structures on $X$, for $n \in \mathbb{Z}$. Here $k(2)[-1]$ is the graded dg-Lie algebra consisting of $k$ in pure cohomological degree 1 , pure weight 2 , and trivial bracket, obviously. If $X$ is a smooth underived scheme, $n=0$, and we replace the mapping space Map $\mathrm{dglie}_{k}^{g r}$ in the model category dglie ${ }_{k}^{g r}$, with its Hom set $H_{o m}$ dglie $_{k}^{g r}$, then we obtain the set of bivectors $\pi$ on $X$, whose Schouten-Nijenhuis self bracket $[\pi, \pi]$ is zero, i.e. exactly the set of Poisson bivectors on $X$. The functoriality problems mentioned above prevent any elementary, easy extension of 
(a shifted version of) the usual Schouten-Nijenhuis bracket when $X$ is a general derived Artin stack, locally of finite presentation over $k$. Hence, there is no elementary, easy way of making sense of the above definition of Poiss $(X, n)$.

Our solution to this problem consists of two steps. First of all, in Section 2.1 we build a very general theory of differential calculus, including de Rham algebras and polyvectors, in an arbitrary symmetric monoidal model $\infty$ category $\mathcal{M}$ enriched over $k$-dg modules (and satisfying suitable, mild hypotheses). In particular, for any commutative algebra $A$ in $\mathcal{M}$, we are able to make sense of the space $\operatorname{Sympl}(A, n)$ of $n$-shifted symplectic structures on $A$, to define a $\mathbb{P}_{n+1}$-commutative differential graded algebra $\operatorname{Pol}(A, n)$ over $k$ of $n$-shifted polyvectors on $A$, and hence to make sense of the space Poiss $(A, n)$ of $n$-shifted Poisson structures on $A$, as explained above. Moreover, we produce a general comparison map from the the space Poiss ${ }^{\text {nd }}(A, n)$, of suitably defined non-degenerate $n$-shifted Poisson structures on $A$ to $\operatorname{Sympl}(A, n)$. The second step is what we call formal localization (Section 2.2), and it concerns essentially the study of the map $X \rightarrow X_{D R}$, for $X$ derived Artin stack, locally of finite presentation over $k$. Here $X_{D R}$ is the de Rham stack of $X$ (Definition 2.2.3), and the fiber of $X \rightarrow X_{D R}$ at a closed point $\bar{x}:$ Spec $\mathbb{K} \rightarrow X_{D R}$ is the formal completion $\widehat{X_{x}}$ of $X$ at the corresponding point $x \in X$ 1; hence $X \rightarrow X_{D R}$ can be viewed as the family of formal completions of $X$.

The remarkable properties of the map $X \rightarrow X_{D R}$, allow us to associate to any derived Artin stack $X$, locally of finite presentation over $k$, a commutative algebra $\mathcal{P}_{X}(\infty)$ in a suitable symmetric monoidal model $\infty$-category $\mathcal{M}_{X}$ constructed out of $X$, such that:

- There is an equivalence of spaces $\operatorname{Sympl}\left(\mathcal{P}_{X}(\infty), n\right) \simeq \operatorname{Symp}(X, n)$.

- After forgetting the bracket $\operatorname{Pol}\left(\mathcal{P}_{X}(\infty), n\right)$ is equivalent to $\operatorname{Pol}(X, n)$, in other words we finally have a way of endowing $\operatorname{Pol}(X, n)$ with the structure of a graded $\mathbb{P}_{n+1}$-commutative differential graded algebra over $k$. In

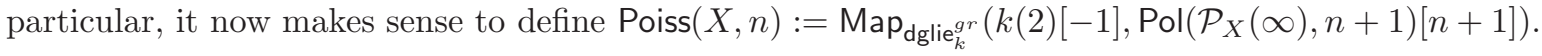

- The canonical map Poiss ${ }^{\mathrm{nd}}(X, n)=\operatorname{Poiss}^{\mathrm{nd}}\left(\mathcal{P}_{X}(\infty), n\right) \rightarrow \operatorname{Sympl}\left(\mathcal{P}_{X}(\infty), n\right) \simeq \operatorname{Symp}(X, n)$ is an equivalence of spaces.

- The $\infty$-category $\operatorname{Perf}(X)$ of perfect complexes on $X$ is equivalent to a suitably defined $\infty$-category of perfect $\mathcal{P}_{X}(\infty)$-dg-modules.

Let us briefly describe the commutative algebra object $\mathcal{P}_{X}(\infty)$ in $\mathcal{M}_{X}$. As already observed, the canonical map $X \longrightarrow X_{D R}$ realizes $X$ as a family of formal derived stacks over $X_{D R}$, namely as the family of formal completions at closed points of $X$. By Lu2 each of these formal completions is determined by a dg-Lie algebra $\ell_{x}$. The collection of dg-Lie algebras $\ell_{x}$ does not fit together globally in a sheaf of dg-Lie algebras over $X_{D R}$, simply because its underlying complex is the shifted tangent complex $\mathbb{T}_{X}[-1]$ of $X$ (see [Hen]), which in general does not admit a flat connection and thus does not descend to $X_{D R}$. However, a remarkable consequence of derived formal localization is that the Chevalley-Eilenberg complexes of $\ell_{x}$, viewed as graded mixed commutative dg-algebras, do fit together into a global object over $X_{D R}$. Up to a twist (by $k(\infty)$, see Section 2.1), this is exactly $\mathcal{P}_{X}(\infty)$ ). Thus, formal localization tells us how to express global geometric objects on $X$ as correspondingly sheafified objects on $X_{D R}$ related to $\mathcal{P}_{X}(\infty)$.

Deformation quantization of derived categories. One of our main original motivations for developing a theory of shifted symplectic and Poisson structures on derived moduli spaces was in fact a prospective application to deformation quantization of derived categories of perfect complexes. We are now able to obtain such applications, and we will briefly describe them here (for more details, see Section 3). We start by defining the deformation quantization problem for $n$ shifted Poisson structures, whenever $n \geq 0$. For every such $n$, we consider a $\mathbb{G}_{m}$-equivariant $\mathbb{A}_{k}^{1}$-family of $k$-dg-operads $\mathbb{B D}_{n+1}$ such that its 0-fiber is the Poisson operad $\mathbb{P}_{n+1}$ and its generic fiber is the $k$-dg-operad $\mathbb{E}_{n+1}$ of chains of the topological operad of little $(n+1)$-disks. The general deformation quantization problem can then be loosely stated as follows:

Deformation Quantization Problem. Given a $\mathbb{P}_{n+1}$-algebra stucture on an object in a k-linear symmetric monoidal $\infty$-categorie, does it exist a family of $\mathbb{B D}_{n+1}$-algebra structures such that its 0 -fiber is the original $\mathbb{P}_{n+1}$ algebra structure?

To be more precise, let now $X$ be a derived Artin stack locally of finite presentation over $k$, and equipped with an $n$-shifted Poisson structure. Using the formality of the $\mathbb{E}_{n}$-operad, and the fact that for $n \geq 1$ the homology operad

\footnotetext{
${ }^{1}$ Note that $X$ and $X_{D R}$ have the same reduced points.
} 
of $\mathbb{E}_{n+1}$ is $\mathbb{P}_{n+1}$, we can solve the deformation quantization problem above for the $\mathbb{P}_{n+1}$-algebra structure on $\mathcal{P}_{X}(\infty)$. This gives us, in particular, a $\mathbb{G}_{m}$-equivariant 1-parameter family of $\mathbb{E}_{n+1}$-algebra structures on $\mathcal{P}_{X}(\infty)$.

One of the main results of formal localization (Section 2.2) tells us that the $\infty$-category $\operatorname{Perf}(X)$ of perfect complexes on $X$ is equivalent to the $\infty$-category of (suitably defined) perfect $\mathcal{P}_{X}(\infty)$-modules (in $\mathcal{M}_{X}$ ). We thus get a 1-parameter deformation of $\operatorname{Perf}(X)$ as an $\mathbb{E}_{n}$-monoidal $\infty$-category, which we call the $n$-quantization of $X$. We also give a version of this result for $n<0$ (where of course $\mathbb{E}_{n}$ will be replaced by $\mathbb{E}_{-n}$ ). In contrast, the unshifted $n=0$ case for derived Artin stacks, which was previously addressed for smooth varieties by [Ko1, Ye, is not currently covered by our analysis and seems to require new ideas.

Finally, in Section 3.2, we describe some examples of these $n$-shifted quantizations, especially the quantization on a formal neighborhood of a point, and of various derived moduli stacks of $G$-local systems, for $G$ a complex reductive group. Many more examples are awaiting a careful investigation.

Acknowledgements. First of all, we would like to thank our co-authors D. Calaque, B. Toën, and M. Vaquié for the interesting mathematics we did together. We thank V. Melani and M. Porta for their interesting questions that have hopefully led to a clearer text. We are grateful to P. Safronov and N. Rozenblyum for useful exchanges on various topics treated in this review. We are also grateful to the organizers of the 2015 Summer Research Institute on Algebraic Geometry in Salt Lake City, for their invitation to give our talks and to write this review.

Tony Pantev was partially supported by NSF research grant DMS-1302242 and by grant \# 347070 from the Simons Foundation. Gabriele Vezzosi is a member of the GNSAGA-INDAM group (Italy) and of PRIN-Geometria delle varietá algebriche (Italy). He would like to point out the serious problems of scientific research in public universities in Italy, due to the substantial lack of acknowledgement from our government of the important cultural and social role of public research in a modern country, and to the subsequent largely insufficient investment of government funds into public research and universities.

Background. We will assume the reader has some familiarity with derived algebraic geometry, for which useful reviews are [To1, and the more recent [To2, while the foundational works are Toën-Vezzosi's [HAG-II], J. Lurie's DAG series [Lu3], and also the recent [Lu5], the last two being available at http://www.math.harvard.edu/ lurie/. We will use both the "old" but sometimes still useful language and theory of model categories (see e.g. [Hov, Hir]), and the modern language and theory of $\infty$-categories ([Lu1, Lu4]).

\section{Notations.}

- Throughout this paper $k$ will denote a noetherian commutative $\mathbb{Q}$-algebra.

- We will use $(\infty, 1)$-categories Lu1 as our model for $\infty$-categories. They will be simply called $\infty$-categories.

- As a general rule, a model category is written in sans-serif fonts $\mathbf{N}$, and we denote in bold-face fonts $\mathbf{N}:=L(\mathbf{N})$ the $\infty$-category defined as the homotopy coherent nerve of the Dwyer-Kan localization of fibrant-cofibrant objects in $\mathrm{N}$ along its weak equivalences, with the notable exceptions of the $\infty$-category of spaces, denoted as $\mathbf{T}:=L(\mathrm{sSets})$, and of our base $\infty$-category $\mathcal{M}:=L(\mathrm{M})$ (Section 2). The passage from a model category to the associated $\infty$-category is a localization, and thus very similar to the passage from the category of complexes in an abelian category to the associated derived category. This is a good example to keep in mind.

- All symmetric monoidal categories we use will be symmetric monoidal (bi)closed categories.

- $\mathrm{dg}_{k}$ will denote the symmetric monoidal model category of (unbounded) complexes of $k$-modules, with fibrations being degreewise surjective amps, and weak equivalences being quasi-isomorphisms. The associated $\infty$-category will be denoted by $\mathbf{d g}_{k}$. Note that $\mathbf{d g}_{k}$ is then a stable symmetric monoidal $\infty$-category ([Lu4, Definition 2.0.0.7]).

- cdga $_{k}$ will denote the $\infty$-category of non-positively graded differential graded $k$-algebras (with differential increasing the degree). Its objects will be frequently called simply cdga's. For $A \in \mathbf{c d g a}_{k}$, we will write $\pi_{i} A:=H^{-i}(A)$ for any $i \geq 0$.

- For $A \in \mathbf{c d g a}_{k}$, we will denote either by $\mathbf{L}(A)$ or $\mathbf{Q C o h}(A)$ the $\infty$-category of $A$-dg-modules.

- For $A \in \mathbf{c d g a}_{k}$, we will denote by $\operatorname{Perf}(A)$ the full sub- $\infty$-category of $\mathbf{Q C o h}(A)$ consisting of perfect $A$-dg-modules.

- If $X$ is a derived geometric stack, we will denote by $\operatorname{QCoh}(X)$ the $k$-linear symmetric monoidal dg-category of quasi-coherent complexes on $X$.

- If $X$ is a derived geometric stack, we will denote by $\operatorname{Perf}(X)$ the symmetric monoidal sub-dg-category of $\mathbf{Q C o h}(X)$ consisting of dualizable objects, i.e. perfect complexes over $X$.

- If $X$ is a derived geometric stack, we will denote by $\operatorname{Coh}(X)$ or the full sub-dg category of $\mathbf{Q} \operatorname{Coh}(X) \operatorname{consisting}$ of complexes whose cohomology sheaves are coherent over the truncation $\mathrm{t}_{0} X$. 
- For a morphism $A \rightarrow B$ of cdga's, the relative cotangent complex will be denoted $\mathbb{L}_{B / A} \in \mathbf{L}(B)$. When $A=k$, we will simply write $\mathbb{L}_{B}$ instead of $\mathbb{L}_{B / k}$.

- For derived stacks, we follow the vocabulary of HAG-II]. In particular derived Artin stacks $X$ will be higher derived stacks, unless stated otherwise, and always have a cotangent complex, denoted as $\mathbb{L}_{X} \in \mathbf{Q C o h}(X)$. The acronym lfp means, as usual, locally finitely presented.

- For a derived stack $X, \Gamma(X,-)$ will always denote the derived functor of global sections on $X$ (i.e. hypercohomology).

\section{Shifted symplectic structures}

\subsection{Definitions}

Let $\epsilon-\mathrm{dg}_{k}^{g r}$ be the category of graded mixed complexes of $k$-dg-modules. Its objects are $\mathbb{Z}$-families of $k$-dg-modules

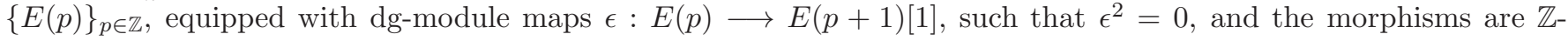
families of morphisms in $\mathrm{dg}_{k}$ commuting with $\epsilon$. This is a symmetric monoidal model category: weak equivalences and cofibrations are defined weight-wise (i.e. with respect to the external $\mathbb{Z}$-grading, that will be called the weight grading), the monoidal structure is defined by $\left(E \otimes E^{\prime}\right)(p):=\bigoplus_{i+j=p} E(i) \otimes E^{\prime}(j)$, and the symmetry constraint does not involve signs, but just swaps the two factors in $E(i) \otimes E^{\prime}(j)$. Since our base ring $k$ has characteristic zero, the category $\operatorname{Comm}\left(\epsilon-\mathrm{dg}_{k}^{g r}\right)=: \epsilon-\operatorname{cdga}_{k}^{g r}$ of commutative monoid objects in $\epsilon-\mathrm{dg}_{k}^{g r}$ is again model category, with weak equivalences and fibrations inherited via the forgetful functor to $\epsilon-\mathrm{dg}_{k}^{g r}$ (which is then a right Quillen adjoint). According to our general conventions, we will denote by $\epsilon-\mathbf{d g}_{k}^{g r}$ (respectively, $\epsilon-\mathbf{c d g a}_{k}^{g r}$ ), the $\infty$-category associated to $\epsilon-\mathrm{dg}_{k}^{g r}$ (respectively to $\epsilon-$ cdga $_{k}^{g r}$ ). Informally speaking, $\epsilon-\mathbf{c d g a}_{k}^{g r}$ is therefore the $\infty$-category of $\left\{B(p) \in \mathbf{d g}_{k}\right\}_{p \in \mathbb{Z}}$ together with mixed differential $\epsilon: B(p) \rightarrow B(p+1)[1], \epsilon^{2}=0$, and maps $B(p) \otimes B(q) \rightarrow B(p+q)$ which are unital, associative, commutative, and suitably compatible with $\epsilon$.

The $\infty$-functor $\epsilon$ - cdga $_{k}^{g r} \rightarrow$ cdga $_{k}:\{B(p)\} \mapsto B(0)$ is accessible and preserves limits, thus (Lu1, Corollary 5.5.2.9]) has a left adjoint DR: $\mathbf{c d g a}_{k} \rightarrow \epsilon-\mathbf{c d g a}_{k}^{g r}$.

Definition 1.1.1 The functor DR: $\mathbf{c d g a}_{k} \rightarrow \epsilon-\mathbf{c d g a}_{k}^{g r}$ is called the de Rham algebra $\infty$-functor.

Remark 1.1.2 If $A \in \mathbf{c d g a}_{k}$, we can replace in the previous argument $\infty$-category $\mathbf{c d g a}_{k}$ with $A / \mathbf{c d g a} \mathbf{a}_{k}$, and the $\infty$-category $\epsilon-\mathbf{c d g a}_{k}^{g r}$ with $\mathbf{D R}(A) / \epsilon-\mathbf{c d g a}_{k}^{g r}$, and get a relative de Rham algebra $\infty$-functor $\mathbf{D R}(-/ A)$.

One can prove that $\mathbf{D R}(A) \simeq \operatorname{Sym}_{A}\left(\mathbb{L}_{A}[-1]\right)$ in $\mathbf{c d g a}_{k}^{g r}$ (i.e. as graded cdga's, by forgetting the mixed differential defined on the rhs). In other words, the construction DR yields the full derived de Rham complex of $A$, including the de Rham differential. We are now able to define shifted closed forms on cdga's. For $m, n \in \mathbb{Z}, k(m)[n]$ will denote the graded $k$-dg-module sitting in weight degree $m$ and in cohomological degree $-n$.

Definition 1.1.3 Let $A \in$ cdga $_{k}$

- The space of closed $n$-shifted $p$-forms on $A$ is $\mathcal{A}^{p, c l}(A, n):=\operatorname{Map}_{\epsilon-\mathbf{d g}_{k}^{g r}}(k(p)[-p-n], \mathbf{D R}(A)) \in \mathbb{T}$. An element in $\pi_{0}\left(\mathcal{A}^{p, c l}(A, n)\right)$ is called a closed $n$-shifted $p$-form on $A$.

- The space of $n$-shifted $p$-forms on $A$ is $\mathcal{A}^{p}(A, n):=\operatorname{Map}_{\mathbf{d g}_{k}}\left(k[-n], \wedge_{A}^{p} \mathbb{L}_{A}\right) \in \mathbb{T}$. An element in $\pi_{0}\left(\mathcal{A}^{p}(A, n)\right)$ is called a $n$-shifted $p$-form on $A$.

- The induced map $u: \mathcal{A}^{p, c l}(A, n) \rightarrow \mathcal{A}^{p}(A, n)$ is called the underlying $p$-form map.

Remark 1.1.4 Here is a more concrete description of the space of shifted (closed) forms. If $A \in \operatorname{cdga}_{k}$, and $A^{\prime} \rightarrow A$ is a cofibrant replacement in $\mathrm{cdga}_{k}$, then $\oplus_{p \geq 0} \mathbb{L}_{A / k}^{p}=\oplus_{p \geq 0} \Omega_{A^{\prime} / k}^{p}$ is a fourth quadrant bicomplex with vertical differential $d^{v}$ induced by $d_{A^{\prime}}$, and horizontal differential $d^{h}$ given by the de Rham differential

$$
d^{v}: \Omega_{A^{\prime} / k}^{p, i} \rightarrow \Omega_{A^{\prime} / k}^{p, i+1}, d^{h}=d_{D R}: \Omega_{A^{\prime} / k}^{p, i} \rightarrow \Omega_{A^{\prime} / k}^{p+1, i} .
$$

The Hodge filtration $F^{\bullet}$ defined by $F^{q}(A):=\oplus_{p \geq q} \Omega_{A^{\prime} / k}^{p}$ is still a fourth quadrant bicomplex, and if we put $\underline{\mathcal{A}}^{p, c l}(A, n):=\operatorname{Tot}^{\Pi}\left(F^{p}(A)[n+p]\right.$, we have

$$
\mathcal{A}^{p, c l}(A, n)=\left|\underline{\mathcal{A}}^{p, c l}(A, n)\right| n \in \mathbb{Z}
$$


where $|E|$ denotes $\operatorname{Map}_{\mathbf{d g}_{k}}(k, E)$ i.e. the Dold-Kan construction applied to the $\leq 0$-truncation of the dg-module $E$, and $\operatorname{Tot}^{\Pi}$ is the totalization by products. In particular, we have a corresponding Hodge tower of dg-modules

$$
\ldots \rightarrow \underline{\mathcal{A}}^{p, c l}(A, 0)[-p] \rightarrow \underline{\mathcal{A}}^{p-1, c l}(A, 0)[1-p] \rightarrow \ldots \rightarrow \underline{\mathcal{A}}^{0, c l}(A, 0),
$$

where, for any $p$, the cofiber of $\underline{\mathcal{A}}^{p, c l}(A, 0)[-p] \rightarrow \underline{\mathcal{A}}^{p-1, c l}(A, 0)[1-p]$ is equivalent to the dg-module $\underline{\mathcal{A}}^{p-1}(A, 0)[1-p]:=$ $\left(\wedge_{A}^{p-1} \mathbb{L}_{A}\right)[1-p]$ of $(1-p)$-shifted $(p-1)$-forms on $\bar{A}$ (so that we have an equivalence $\left|\underline{\mathcal{A}}^{p-1}(A, 0)[1-p]\right| \simeq \mathcal{A}^{p-1}(A, 1-p)$ in $\mathbf{T})$. Finally, let us observe that the rightmost dg-module $\underline{\mathcal{A}}^{0, c l}(A, 0)$ in the above Hodge tower, is exactly Illusie's derived de Rham complex of $A$ ([III, ch. VIII]).

Remark 1.1.5 Note that the de Rham algebra functor, and hence the notion of (closed) shifted forms, makes sense when $\mathbf{d g}_{k}$ is replaced by (essentially) any symmetric monoidal stable $k$-linear $\infty$-category $\mathcal{M}$. The intermediate categories of interest will then be $\epsilon-\mathcal{M}^{g r}$ (generalizing $\epsilon-\mathbf{d g}_{k}^{g r}$ ), and $\epsilon-\mathbf{C A} \lg _{\mathcal{M}}^{g r}$ (generalizing $\epsilon-\operatorname{cdga}_{k}^{g r}$ ). For any $A \in \mathbf{C A l g}{ }_{\mathcal{M}}$, this will yield a cotangent complex $\mathbb{L}_{A}^{\mathcal{M}} \in A-\operatorname{Mod}_{\mathcal{M}}$, a de Rham algebra functor $\mathbf{D R}{ }^{\mathcal{M}}: \mathbf{C A l g}_{\mathcal{M}} \rightarrow$ $\epsilon-\mathbf{C A} \lg _{\mathcal{M}}^{g r}$, and a space of $n$-shifted (closed) $p$-form $\mathcal{A}_{\mathcal{M}}^{p}(A, n)\left(\mathcal{A}_{\mathcal{M}}^{p, c l}(A, n)\right)$, where the sub/superscript $\mathcal{M}$ indicates that all the constructions are performed internally to $\mathcal{M}$. This level of generality and flexibility in the choice of the context for our differential calculus, will prove extremely useful in the rest of the paper. As relevant cases, the reader should keep in mind the case where $\mathcal{M}=\epsilon-\mathrm{dg}_{k}^{g r}$ or, more generally, diagrams in $\epsilon-\mathrm{dg}_{k}^{g r}$. We will come back to this generalization more systematically in Section 2.1, and use it as an essential tool starting from Section 2.3

We are now ready to globalize the above construction to derived stacks. We start by globalizing the de Rham algebra construction (Definition 1.1.1). The functor $A \rightarrow \mathbf{D R}(A)$, and its relative version (over a fixed base $B$, see Remark 1.1.2), are both derived stacks (for the étale topology) with values in mixed graded dga's, so we give the following

Definition 1.1.6 (1) Let $F \rightarrow \mathbf{S p e c} B$ be a map in $\mathbf{d S t}_{k}$. The relative de Rham algebra of $F$ over $B$

$$
\mathbf{D R}(F / B):=\lim _{\mathbf{S p e c} C \rightarrow F} \mathbf{D R}(C / B) \in \epsilon-\mathbf{c d g a}_{B}^{g r}
$$

(see Remark 1.1.2) where the limit is taken in the $\infty$-category $\epsilon-\mathbf{c d g a}_{B}^{g r}=B / \epsilon-\mathbf{c d g a}_{k}^{g r}$ of graded mixed B-linear cdgas, and over all morphisms $\mathbf{S p e c} C \rightarrow F$ of derived stacks over $\mathbf{S p e c} B$.

(2) For an arbitrary map $F \rightarrow G$ in $\mathbf{d S t}_{k}$, we define the relative de Rham algebra of $F$ over $G$ as

$$
\mathbf{D R}(F / G):=\lim _{\mathbf{S p e c} A \rightarrow G} \mathbf{D R}\left(F_{A} / A\right) \in \epsilon-\mathbf{c d g a}_{k}^{g r},
$$

where $F_{A}$ denotes the base change of $F \rightarrow G$ along $\mathbf{S p e c} A \rightarrow G$, and the limit is taken in the $\infty$-category $\epsilon-\mathbf{c d g a}_{k}^{g r}$.

We now globalize to derived stacks the notion of (closed) shifted forms. From Definition [1.1.3 one deduces $\infty$ functors $\mathcal{A}^{p, c l}(-, n): A \mapsto \mathcal{A}^{p, c l}(A, n)$, and $\left.\mathcal{A}^{p}(-, n)\right): A \mapsto \mathcal{A}^{p}(A, n)$ from cdga ${ }_{k}$ to T. By [PTVV Proposition 1.11], these functors are derived stacks (for the étale topology). This allows us to globalize Definition 1.1.3 on an arbitrary derived stack.

Definition 1.1.7 Let $F$ be a derived stack.

- The space of closed $n$-shifted $p$-forms on $F$ is $\mathcal{A}^{p, c l}(F, n):=\operatorname{Map}_{\mathbf{d S t}_{k}}\left(F, \mathcal{A}^{p, c l}(-, n)\right)$.

- The space of $n$-shifted $p$-forms on $F$ is $\mathcal{A}^{p}(F, n):=\operatorname{Map}_{\mathbf{d S t}_{k}}\left(F, \mathcal{A}^{p}(-, n)\right)$.

- The resulting induced map $u: \mathcal{A}^{p, c l}(F, n) \rightarrow \mathcal{A}^{p}(F, n)$ is called the underlying $p$-form map.

Note that, in general, the homotopy fiber of the underlying $p$-form map $u$ can be non-trivial (i.e. not empty nor contractible). Hence being closed is a datum rather than a property, for a general derived stack. 
Remark 1.1.8 (1) Equivalently, we have $\mathcal{A}^{p, c l}(F, n) \simeq \lim _{\mathbf{S p e c} A \in(\mathbf{d A f f} / F)^{o p}} \mathcal{A}^{p, c l}(A, n)$, and $\mathcal{A}^{p}(F, n)$ $\simeq \lim _{\mathbf{S p e c} A \in(\mathbf{d A f f} / F)^{o p}} \mathcal{A}^{p, c l}(A, n)$, where the limits are taken in the $\infty$-category of $\infty$-functors from $(\mathbf{d} \mathbf{A f f} / F)^{o p}$ to $\mathbf{T}$.

(2) Also note that we have an equivalence $\mathcal{A}^{p, c l}(F, n) \simeq \operatorname{Map}_{\epsilon-\mathbf{d g}_{k}^{g r}}(k(p)[-p-n], \mathbf{D R}(F / k))$ in $\mathbf{T}$.

(3) For $F=\operatorname{Spec} A$, the complex $\wedge^{p} \mathbb{L}_{A}$ has non-positive tor-amplitide, hence there are no non-trivial $n$-shifted $p$-forms on $F$, for $n>0$. For $F=X$ an underived smooth scheme, a similar argument shows that $F$ admits no non-trivial $n$-shifted $p$-forms on $F$, for $n<0$. If moreover $X$ is proper over $k$, then the degeneration of the Hodge-to-de Rham spectral sequence implies that the underlying $p$-form map is injective on $\pi_{0}$.

For derived (higher) Artin stacks $F$, the space $\mathcal{A}^{p}(F, n)$ has the following equivalent description (smooth descent).

Proposition 1.1.9 PTVV, Proposition 1.14] If $F \in \mathbf{d S t}$ is Artin, then we have an equivalence

$$
\mathcal{A}^{p}(F, n) \simeq \operatorname{Map} \operatorname{QCoh}(F)_{F}\left(\mathcal{O}_{F}, \wedge^{p} \mathbb{L}_{F}[n]\right),
$$

functorial in F.

In particular $\pi_{0}\left(\mathcal{A}^{p}(F, n)\right) \simeq \mathbb{H}^{n}\left(X, \wedge^{p} \mathbb{L}_{F}\right)$, for $F$ Artin. Thus an $n$-shifted 2-form $\omega$ can be identified with a map $\omega: \mathcal{O}_{F} \rightarrow \wedge^{2} \mathbb{L}_{F}[n]$. If $F$ is moreover locally of finite presentation over $k$ (so that its cotangent complex $\mathbb{L}_{F}$ is perfect, i.e. dualizable in $\mathbf{Q C o h}(F)$ ), we may associate to such an $\omega$ an adjoint map $\omega^{b}: \mathbb{T}_{F} \rightarrow \mathbb{L}_{F}[n]$, where $\mathbb{T}_{F}$ denotes the dual of $\mathbb{L}_{F}$ in $\mathbf{Q C o h}(F)$, and it is called the tangent complex of $F$. An $n$-shifted 2 form $\omega$ on such an $F$ is said to be non-degenerate if the map $\omega^{b}$ is an equivalence, i.e. an isomorphism in the derived category of quasi-coherent complexes on $F$, and we will denote by $\mathcal{A}_{n d}^{2}(F, n)$ the subspace of $\mathcal{A}^{2}(F, n)$ consisting of connected components of non-degenerate forms.

Definition 1.1.10 Let $F$ be an derived Artin stack locally of finite presentation over $k$. The space of $n$-shifted symplectic structures on $F$ is defined by the following pullback diagram in $\mathbb{T}$

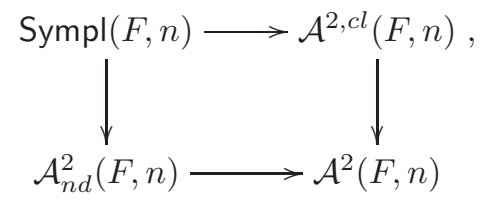

and an element in $\pi_{0}(\operatorname{Sympl}(F ; n))$ is called a $n$-shifted symplectic structure on $F$.

In other words, an $n$-shifted symplectic structure $\omega$ on $F$ is a closed $n$-shifted 2 -form on $F$ whose underlying 2 -form is non-degenerate; in particular, $\mathbf{L}_{F}$ is self dual, up to a shift. We use the word symplectic structure instead of symplectic form because a shifted symplectic structure is a closed-2-form, and with respect to the underlying form, this consists of additional structure rather than just being a property.

The non-degeneracy condition entails a mixture of the (higher) stacky (i.e. positive degrees in the cotangent complex) and derived (i.e. negative degrees of the cotangent complex) nature of the stack $F$, and in particular it poses severe restrictions on the existence of shifted symplectic structures on a given stack. E.g. it is clear that if $\mathbb{L}_{F}$ has perfect amplitude in $[a, b]$, then $F$ may only support $(a+b)$-shifted symplectic structures. More precisely, it is easy to check that for a smooth underived scheme $X$, not étale over $k$, the space $\operatorname{Sympl}(X, n)$ is empty for $n \neq 0$, and either empty or contractible for $n=0$, and moreover, the set of connected components of $\operatorname{Sympl}(X, 0)$ is in canonical bijection with the set of usual algebraic symplectic forms on $X$ ([PTVV] p. 298]). So, we get nothing new for smooth underived schemes, or more generally, smooth Deligne-Mumford stacks. However, we will see in the following subsection that there are plenty of derived schemes or stacks carrying interesting shifted symplectic forms. 


\section{$1.2 \quad$ Existence theorems and examples}

We will now review the three basic existence theorems (Theorems 1.2.1, 1.2.5, 1.2.8, below) for shifted symplectic structures established in PTVV]. In combination they give a long list of non-trivial examples of shifted symplectic stacks.

The first interesting example of a shifted symplectic stack is the classifying stack $B G$ of a smooth affine reductive group scheme over $k$. In this case, we have ([PTVV p. 299])

$$
\pi_{0}(\operatorname{Sympl}(\mathrm{B} G, n))= \begin{cases}0 & n \neq 2 \\ \operatorname{Sym}_{k}\left(\mathfrak{g}^{\vee}\right)_{n d}^{G} & n=2\end{cases}
$$

where $\mathfrak{g}$ is the Lie algebra of $G$, and $\operatorname{Sym}_{k}\left(\mathfrak{g}^{\vee}\right)_{n d}^{G}$ is the set of non-degenerate $G$-invariant symmetric bilinear forms on $\mathfrak{g}$. At the level of tangent complex $\mathbb{T}_{\mathrm{B} G, e} \simeq \mathfrak{g}[1]$, the underlying 2-form corresponding to a non-degenerate $G$-invariant symmetric bilinear form $\sigma: \operatorname{Sym}_{k}^{2}(\mathfrak{g}) \rightarrow k$ is given by the composite

$$
\mathbb{T}_{\mathrm{B} G, e} \wedge \mathbb{T}_{\mathrm{B} G, e} \stackrel{\sim}{\longrightarrow} \mathfrak{g}[1] \wedge \mathfrak{g}[1] \stackrel{\sim}{\longrightarrow} \operatorname{Sym}_{k}^{2}(\mathfrak{g})[2] \stackrel{\sigma[2]}{\longrightarrow} k[2],
$$

where the central quasi-isomorphism is given by décalage. For example, if $G=\mathrm{GL}_{n}$, the usual map $(A, B) \mapsto \operatorname{tr}(A B)$, for $A, B(n \times n)$ matrices over $k$, yields a 2 -shifted symplectic form on $\mathrm{BGL}_{n}$. This example will be vastly generalized in Theorem 1.2 .8 below.

As a second example, for any $n \in \mathbb{Z}$, one has that the $n$-shifted cotangent stack $\mathrm{T}^{*} F[n]:=$ $\operatorname{Spec}_{F}\left(\operatorname{Sym}_{\mathcal{O}_{F}}\left(\mathbb{T}_{F}[-n]\right)\right)$ of a derived Deligne-Mumford stack $F$ lfp over $k$, is canonically $n$-shifted symplectic via the de Rham differential of the canonical shifted Liouville form ([PTVV, Proposition 1.21]). Recently, D. Calaque has extended this result to derived Artin stacks lfp over $k$ Cal2].

The first general existence result for shifted symplectic form is an enhanced derived version of the main result in AKSZ.

Theorem 1.2.1 Let $F$ be a derived Artin stack lfp over $k$, equipped with a $n$-shifted symplectic form, and let $X$ be an $\mathcal{O}$-compact derived stack equipped with a d-orientation $[X]: \mathbb{H}\left(X, \mathcal{O}_{X}\right) \rightarrow k[-d]$. If $\operatorname{MAP}_{\mathrm{dSt}}(X, F)$ is a derived Artin stack lfp over $k$, then it carries a canonical $(n-d)$-shifted symplectic structure.

We direct the reader to [PTVV] 2.1] for the definition of $\mathcal{O}$-compact derived stack, and for the notion of $d$-orientation on a $\mathcal{O}$-compact derived stack (i.e. for the special properties of the map $[X]$ in the theorem), and to PTVV, Theorem 2.5] for a detailed proof. Here we will content ourselves with a few comments.

First of all the class $\mathcal{O}$-compact derived stacks equipped with a $d$-orientation includes compact smooth oriented topological manifolds $M$ of dimension $d$ (identified with constant stacks with value $M$, and where capping with the fundamental class gives the $d$-orientation), Calabi-Yau varieties of complex dimension $d$ (where the orientation is given by a trivialization of the canonical sheaf followed by the trace map), and De Rham stacks $X=Y_{D R}$ for $Y$ a smooth and proper Deligne-Mumford stack with connected geometric fibers, and relative dimension $d / 2$ over Spec $k 2$ (where the $d$-orientation is induced by the choice of a fundamental class in de Rham cohomology $\mathbb{H}_{d R}(Y / k, \mathcal{O})$ ).

As a second comment we outline the proof of Thm 1.2.1. One first uses the evaluation map ev : $X \times \operatorname{MAP}_{\mathrm{dSt}}(X, F) \rightarrow$ $F$ in order to pull back the $n$-shifted symplectic structure $\omega$ on $F$, to a closed form on $X \times \operatorname{MAP}_{\mathbf{d S t}}(X, F)$; this pullback is then "integrated along the fiber" of the projection $X \times \operatorname{MAP}_{\mathbf{d S t}}(X, F) \rightarrow \operatorname{MAP}_{\mathbf{d S t}}(X, F)$, and this integrated form is shown to be $(n-d)$-shifted symplectic. The possibility of defining an integration along the fiber $X$ is a consequence of the definition of $d$-orientation on an $\mathcal{O}$-compact stack ([PTVV, Definition 2.3]). Finally, we observe that the general question of finding optimal conditions on $X$ and $F$ ensuring that $\operatorname{MAP}_{\mathbf{d S t}}(X, F)$ is a derived Artin stack lfp over $k$ is delicate, the Artin-Lurie representability criterion (even in the simplified version of [HAG-II, Appendix]) will give an answer in all the applications we will discuss below.

We come to the second existence theorem for shifted symplectic structures. Before stating it, we need to define the notion of lagrangian structure on a map whose target is a shifted symplectic stack. We start by defining what is an isotropic structure on such a map.

Definition 1.2.2 Let $X$ and $F$ be derived Artin stacks lfp over $k, \omega$ a n-shifted symplectic structure on $F$, and $f: X \rightarrow F$ a map. The space $\operatorname{Isotr}(f ; \omega)$ of isotropic structures on the map $f$ relative to $\omega$ is the space of paths $\Omega_{f^{*} \omega, 0} \mathcal{A}^{2, c l}(X, n)$ between $f^{*} \omega$ and 0 in the space of $n$-shifted closed $p$-forms on $X$. An element in $\gamma \in \pi_{0}(\operatorname{Isotr}(f ; \omega))$, i.e. a path between $f^{*} \omega$ and 0 , is called an isotropic structure on $f$ relative to $\omega$.

\footnotetext{
${ }^{2}$ The notion of de Rham stack will be defined and discussed in Section 2.2
} 
The idea, ubiquitous in all of derived geometry and higher category theory, and that we already saw in action in the definition of closed forms (Def 1.1.3), is that it is not enough to say that there exists a path between $f^{*} \omega$ and 0 in $\mathcal{A}^{2, c l}(X, n)$ (this would be a property), but one rather has to specify one such path (i.e. a datum).

Once an isotropic structure $\gamma$ is given, it makes sense to say that it has the property of being non-degenerate, as follows. By composition with the underlying 2-form map $u: \mathcal{A}^{2, c l}(X, n) \rightarrow \mathcal{A}^{2}(X, n)$, the path $\gamma$ induces a path $\gamma_{u}$ between $u\left(f^{*} \omega\right)=f^{*}(u(\omega))$ and 0 in $\mathcal{A}^{2}(X, n)$. Since $\mathbb{L}_{X}$ is perfect, by adjunction, this yields in turn a path $\gamma_{u}^{b}$ between $\left(f^{*}(u(\omega))\right)^{b}$ and 0 in $\operatorname{Map} \operatorname{Perf}_{\operatorname{P}(X)}\left(\mathbb{T}_{X}, \mathbb{L}_{X}[n]\right)$, where $\left(f^{*}(u(\omega))\right)^{b}$ is the composite

$$
\mathbb{T}_{X} \stackrel{a^{\vee}}{\longrightarrow} f^{*} \mathbb{T}_{F} \stackrel{f^{*}\left(u(\omega)^{b}\right)}{\longrightarrow} f^{*} \mathbb{L}_{F}[n] \stackrel{a[n]}{\longrightarrow} \mathbb{L}_{X}[n],
$$

$a: f^{*} \mathbb{L}_{F} \rightarrow \mathbb{L}_{X}$ being the canonical map induced by $f$. If we denote by $t_{f, \omega}$ the composite $a[n] \circ f^{*}\left(u(\omega)^{b}\right)$, we thus obtain that $\gamma_{u}^{b}$ is a homotopy commutativity datum for the square

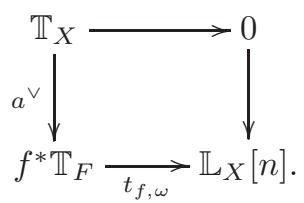

In particular, if we denote by $\mathbb{T}_{f, \omega}^{+}$the pullback in the diagram

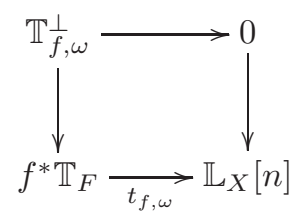

(i.e. the kernel of $t_{f, \omega}$ ), we get a canonical induced map $\theta_{\gamma}: \mathbb{T}_{X} \rightarrow \mathbb{T}_{f, \omega}^{\perp}$ in $\operatorname{Perf}(X)$.

Definition 1.2.3 In the setting of Definition 1.2.2, an isotropic structure $\gamma \in \pi_{0}(\operatorname{Isotr}(f ; \omega))$ is called non-degenerate or lagrangian if the induced map $\theta_{\gamma}: \mathbb{T}_{X} \rightarrow \mathbb{T}_{f, \omega}^{\perp}$ is an equivalence in $\operatorname{Perf}(X)$ (i.e. an isomorphism in the underlying derived/homotopy category). The space $\operatorname{Lagr}(f ; \omega)$ of lagrangian structures on $f: X \rightarrow F$ relative to $\omega$ is the subspace of Isotr $(f ; \omega)$ consisting of connected components of lagrangian structures.

Remark 1.2.4 (1) It is easy to check that if $X$ and $F$ are underived smooth schemes, $\omega$ is a usual (i.e. 0-shifted) symplectic structure on $F$, and $f$ is a closed immersion, then $\operatorname{Lagr}(f ; \omega)$ is either empty or contractible, and it is in fact contractible iff $X$ is a usual smooth lagrangian subscheme of $F$ via $f$. The nondegeneracy condition ensures that the dimension of $X$ is then half of the dimension of $F$, and in fact $\mathbb{T}_{f, \omega}^{\perp}$ is then quasi-isomorphic to the usual symplectic orthogonal of $\mathbb{T}_{X}=\mathrm{T}_{X}$ in $\mathbb{T}_{F}=\mathrm{T}_{X}$. Thus, the notion of lagrangian structure reduces to the usual notion of lagrangian subscheme in this case.

(2) By rephrasing Definition 1.2.3, an isotropic structure $\gamma \in \pi_{0}(\operatorname{Isotr}(f ; \omega))$ is lagrangian iff the (homotopy) commutative square

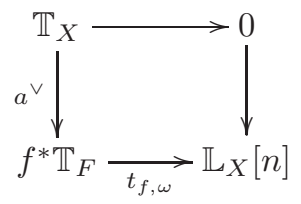

is actually a pullback square. But the square

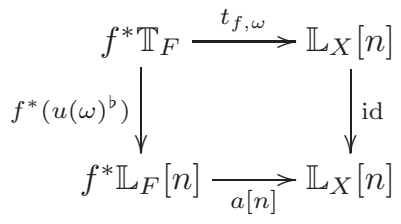


is a pullback because $f^{*}\left(u(\omega)^{b}\right)$ is an equivalence $(u(\omega)$ being non-degenerate), hence $\gamma$ is lagrangian iff the outer square in

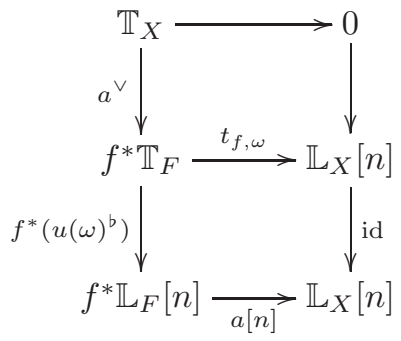

is cartesian, i.e. iff the induced canonical map $\rho_{\gamma}: \mathbb{T}_{X} \rightarrow \operatorname{ker}(a[n]) \simeq \mathbb{L}_{f}[n-1]$ is an equivalence i.e. iff the shifted dual map $\Theta_{\gamma}:=\rho_{\gamma}^{\vee}[n-1]: \mathbb{T}_{f} \rightarrow \mathbb{L}_{X}[n-1]$ is an equivalence. This shows the equivalence between Definition 1.2 .3 and [PTVV, Definition 2.8].

(3) As first noticed by D. Calaque, shifted symplectic structures are particular instances of lagrangian structures (a fact that is obviously false inside usual, 0 -shifted and underived algebraic geometry). In fact, let $n \in \mathbb{Z}, X$ be a derived stack lfp over $k, f: X \rightarrow$ Spec $k$ be the structure map, and let us endow $\operatorname{Spec} k$ with the unique $(n+1)$-shifted symplectic structure $\omega_{n+1}^{0}$ (note that $\mathbb{L}_{k} \simeq \Omega_{k / k}^{1}[0] \simeq 0[0]$, therefore for any $m \in \mathbb{Z}$, there is a unique $m$-shifted symplectic form given by the the shift of the zero form). Now, if $\gamma \in \pi_{0}(\operatorname{Lagr}(f ; \omega))$ as in Definition 1.2.3 then $\gamma$ is actually a loop at 0 inside $\mathcal{A}^{2, c l}(X, n+1)$, and its class $[\gamma] \in \pi_{1}\left(\Omega_{0,0}\left(\mathcal{A}^{2, c l}(X, n+1)\right)\right) \simeq \pi_{0}\left(\mathcal{A}^{2, c l}(X, n)\right) \sqrt{3}$ gives an $n$-shifted closed 2-form $\omega_{\gamma}$ on $X$. The non-degeneracy condition on $\gamma$ is equivalent to the fact that the induced map $\Theta_{\gamma}: \mathbb{T}_{f} \simeq \mathbb{T}_{X} \rightarrow \mathbb{L}_{X}[n-1]$ of point (2) in this Remark, is an equivalence. But it is easy to check that this map is exactly $\omega_{\gamma}^{b}$, hence $\omega_{\gamma}$ is indeed an $n$-shifted symplectic form on $X$. By using again that $\pi_{1}\left(\Omega_{0,0}\left(\mathcal{A}^{2, c l}(X, n+1)\right)\right) \simeq \pi_{0}\left(\mathcal{A}^{2, c l}(X, n)\right)$, and running the previous argument backwards, we get an equivalence of spaces $\operatorname{Sympl}(X, n) \simeq \operatorname{Lagr}\left(f: X \rightarrow \operatorname{Spec} k, \omega_{n+1}^{0}\right)$.

The link between shifted symplectic structures and lagrangian structures expressed in Remark 1.2.4 (3) extends to the case of lagrangian intersections as follows.

Theorem 1.2.5 Let $n \in \mathbb{Z},(F, \omega)$ be a n-shifted symplectic stack, $f_{i}: X_{i} \rightarrow F, i=1,2$ maps between derived Artin stacks lfp over $k$, and $\gamma_{i}$ lagrangian structures on $f_{i}$ relative to $\omega, i=1,2$. Then, there is a canonical induced $(n-1)$-shifted symplectic structure on the fiber product $X_{1} \times_{F} X_{2}$.

Recall that, according to our conventions, all fiber products of derived stacks are taken in the $\infty$-category of derived stacks, and are therefore implicitly derived fiber products. We refer the reader to [PTVV] Theorem 2.9] for a proof of Theorem 1.2.5. We will only give a sketch of the argument in the classical case i.e. for $n=0, F, X_{1}, X_{2}$ underived smooth schemes, and $f_{i}$ closed immersions, $i=1,2$, in order to convey the main idea of why Theorem 1.2 .5 is true. Under our hypotheses, $X_{1}$ and $X_{2}$ are usual lagrangian smooth subschemes of the smooth symplectic scheme $F$. If $X_{12}:=X_{1} \times_{F} X_{2}$ denotes the (derived) intersection of $X_{1}$ and $X_{2}$ inside $F$, we may pull back the closed form $\omega$ to $X_{12}$ in two different ways, i.e. using $f_{1}$ or $f_{2}$, and get two closed forms $\omega_{1}$ and $\omega_{2}$ on $X_{12}$. Now, $X_{12}$ is a derived fiber product, hence these two pullbacks come equipped with a canonical path $\gamma$ between them inside $\mathcal{A}^{2, c l}\left(X_{12}, 0\right)$. On the other hand, as $X_{1}$ and $X_{2}$ are lagrangian subschemes we have $\omega_{1}=\omega_{2}=0$, so that $\gamma$ is in fact a loop at 0 in $\mathcal{A}^{2, c l}\left(X_{12}, 0\right)$. Since, $\pi_{1}\left(\mathcal{A}^{2, c l}\left(X_{12}, 0\right) ; 0\right) \simeq \pi_{0}\left(\mathcal{A}^{2, c l}\left(X_{12},-1\right)\right)$, the class $[\gamma] \in \pi_{0}\left(\mathcal{A}^{2, c l}\left(X_{12},-1\right)\right)$ defines a $(-1)$-shifted closed 2 -form on $X_{12}$, whose non-degeneracy follows easily from the same property for $\omega$. Thus $[\gamma]$ is a $(-1)$-shifted symplectic structure on the derived intersection $X_{12}$. The appearence of a (-1)-shift here perhaps explains why this phenomenon, even though arising from a completely classical situation in usual (algebraic) symplectic geometry, was not observed before.

Remark 1.2.6 The following special case of 1.2.5 is particularly relevant for applications to Donaldson-Thomas invariants (see Theorem 1.2.10 below). Let $X$ be a smooth underived scheme and $g: X \rightarrow \mathbb{A}_{k}^{1}$ a smooth function. We may embed $X$ inside its cotangent bundle either via the zero section or via the differential $d g: X \rightarrow \mathrm{T}^{*} X$, and both these immersions are lagrangian with respect to the standard symplectic structure on $\mathrm{T}^{*} X$. The derived intersection of these two lagrangians is called the derived critical locus of $g$, and is denoted by dCrit $(g)$. Note that its truncation is the usual scheme-theoretic critical locus of $g$. Now, Theorem 1.2 .5 endow $\mathrm{dCrit}(g)$ with a canonical

\footnotetext{
${ }^{3}$ This isomorphism is perhaps more familar to the reader in the following form: a self-homotopy $h$ of the zero map of complexes $0: E \rightarrow F$ is the same thing as map of complexes $E \rightarrow F[1]$.
} 
$(-1)$-shifted symplectic structure $\omega_{g}$. One can rather easily give an explicit description of $\omega_{g}$ using Koszul resolutions (see, e.g. Vez1]), and observe that a similar result holds by replacing $d g$ with an arbitrary closed 1-form on $X$. However, since derived critical loci are particularly important as local models of $(-1)$-shifted symplectic structures, the generalization to derived zero schemes of closed 1-forms has not yet received much attention. Also notice that the previous construction can be easily generalized to build derived zero loci of sections of arbitrary locally free sheaves on $X$.

Remark 1.2.7 One may combine Theorem 1.2.1 and Definition 1.2.3 together with a notion of relative orientation, in order to get a relative version of Theorem 1.2.1. This is due to D. Calaque ([Cal, 2.3]). Here is an outline of the construction. Given a map of derived stacks $b: B \rightarrow X$, and a perfect complex $E$ on $X$, we let $\mathbb{H}(X \operatorname{rel} B, E)$ be the fiber of the induced map $b_{E}: \Gamma(X, E) \rightarrow \Gamma\left(B, b^{*} E\right)$. We define a relative $d$-orientation on $b$ is a map $\eta_{b}: \mathbb{H}\left(X \operatorname{rel} B, \mathcal{O}_{X}\right) \rightarrow k[-d]$ satisfying the following two non-degeneracy properties. First of all, we assume that $B$ is $\mathcal{O}$-compact, and we require that the composite map $\Gamma\left(B, \mathcal{O}_{B}\right) \rightarrow \mathbb{H}\left(X \operatorname{rel} B, \mathcal{O}_{X}\right)[1] \rightarrow k[-d+1]$ defines a $(d-1)$ orientation on $B$. Then, for any $E \in \operatorname{Perf}(X)$, we ask that the induced map

$$
\Gamma(X, E) \otimes \mathbb{H}\left(X \operatorname{rel} B, E^{\vee}\right) \stackrel{\alpha}{\longrightarrow} \mathbb{H}\left(X \operatorname{rel} B, \mathcal{O}_{X}\right) \stackrel{\eta_{b}}{\longrightarrow} k[-d]
$$

yields, by adjunction, an equivalence $\Gamma(X, E) \simeq \mathbb{H}\left(X \operatorname{rel} B, E^{\vee}\right)[-d]$. Here $\alpha$ is the map induced on the vertical fibers of

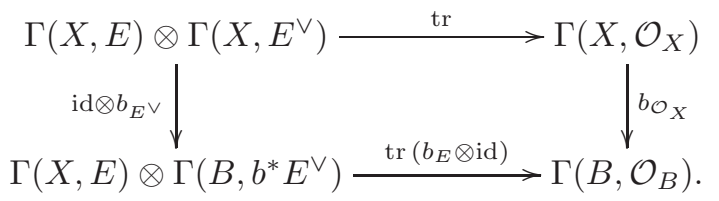

Given a $d$-orientation $\eta_{b}$ on the map $b: B \rightarrow X$, a $(n+1)$-shifted symplectic stack $Y^{\prime}$, a map $\ell: Y \rightarrow Y^{\prime}$, and a lagrangian structure on $f$, we may consider the derived stack

$$
\operatorname{MAP}_{\mathbf{d S t}}(b, f):=\operatorname{MAP}_{\mathbf{d S t}}(B, Y) \times \operatorname{MAP}_{\mathbf{d S t}}\left(B, Y^{\prime}\right) \operatorname{MAP}_{\mathbf{d S t}}\left(X, Y^{\prime}\right)
$$

of arrows from $b$ to $f$. The generalization of Theorem 1.2.1 to this relative situation says that if $\operatorname{MAP}_{\mathbf{d S t}}(b, \ell)$ and $\operatorname{MAP}_{\mathbf{d S t}}(X, Y)$ are derived Artin stacks lfp over $k$, then $\operatorname{MAP}_{\mathbf{d S t}}(b, \ell)$ has a canonical $(n-d+1)$-shifted symplectic form, and there is a canonical lagrangian structure on the natural map $\operatorname{MAP}_{\mathbf{d S t}}(X, Y) \rightarrow \operatorname{MAP}_{\mathbf{d S t}}(b, \ell)$. If we take $B$ to be empty (so that $\eta_{b}$ is just a $d$-orientation on $X$ ), and $Y=\left(\operatorname{Spec} k, \omega_{n+1}^{0}\right)$, we have $\operatorname{MAP}_{\mathbf{d S t}}(b, \ell) \simeq\left(\operatorname{Spec} k, \omega_{n-d+1}^{0}\right)$, and by Remark 1.2.4(3), we get back Theorem 1.2.1 But we may also take $Y=\left(\operatorname{Spec} k, \omega_{n+1}^{0}\right)$, and $B$ arbitrary (nonempty), and we get a lagrangian structure on the restriction map $\operatorname{MAP}_{\mathbf{d S t}}(X, Y) \rightarrow \operatorname{MAP}_{\mathbf{d S t}}(B, Y) \simeq \operatorname{MAP}_{\mathbf{d S t}}(b, \ell)$, where $\operatorname{MAP}_{\mathbf{d S t}}(B, Y)$ is $(n-d+1)$-symplectic (consistently with Theorem 1.2.1, since $B$ is $(d-1)$-oriented by hypothesis, and $Y$ is $n$-shifted symplectic by Remark 1.2.4(3)). In other words, what we gain in this case, is that restriction to the boundary (for maps to a fixed shifted symplectic target) is endowed with a lagrangian structure. Examples of relative orientations includes topological examples (where $b$ is the inclusion of the boundary in a compact oriented topological $d$-manifold with boundary), and algebro-geometric ones where $b$ is the inclusion of the derived zero locus (Remark 1.2.6) of a section of the anti-canonical bundle of a smooth projective variety of dimension $d$. When $B$ is a $K 3$ surface inside a Fano 3-fold, this might be compared with [Ty, Proposition 2.2]. For more details, we address the reader to [Cal, 3.2.2], and [To2, p. 227].

The last general existence theorem for shifted symplectic structures is a generalization of the 2-shifted symplectic structure on $\mathrm{BGL}_{n}$ described at the beginning of this subsection.

Let Perf be the derived stack classifying perfect complexes. It can be defined as the functor sending a cdga $A$ to the nerve of the category of cofibrant perfect $A$-dg-modules with morphisms only the quasi-isomorphisms (as an $\infty$-functor it sends $A$ to the coherent nerve of the Dwyer-Kan localization of the previous category). The truncation of Perf is the (higher) stack first introduced and studied in [HS. Though Perf is not strictly speaking a derived Artin stack lfp over $k$, it is quite close to it: it is locally geometric, i.e. it is a union of open derived Artin substacks lfp over $k$, e.g. Perf $\simeq \cup_{n \geq 0} \operatorname{Perf}^{[-n, n]}$, where Perf ${ }^{[-n, n]}$ is the derived stack classifying perfect complexes of Tor-amplitude contained in $[-n, n]$ (To-Va Proposition 3.7]). In particular, it makes sense to ask wether Perf carries a shifted symplectic structure, as we know its substack Perf ${ }^{[-0,0]}=\mathrm{BGL} \simeq \bigsqcup_{n \geq 0} \mathrm{BGL}_{n}$ does. As shown in [PTVV], Theorem 2.12], the answer is affirmative. 
Theorem 1.2.8 The derived stack Perf has a canonical 2-shifted symplectic structure.

We will briefly give an idea of the proof, and address the readers to [PTVV, Theorem 2.12] for all details. First of all, by definition, Perf carries a universal perfect complex $\mathcal{E}$, and we consider its perfect $\mathcal{O}_{\text {Perf }}$-Algebra of endomorphisms $\mathcal{B}:=\mathbb{R} \underline{E n d}_{\mathcal{O}_{\text {Perf }}}(\mathcal{E}) \simeq \mathcal{E}^{\vee} \otimes_{\mathcal{O}_{\text {Perf }}} \mathcal{E}$. One checks that $\mathbb{T}_{\text {Perf }} \simeq \mathcal{B}[1]$, and thus gets a well defined map

$$
\omega^{0}: \mathbb{T}_{\text {Perf }} \wedge_{\mathcal{O}_{\text {Perf }}} \mathbb{T}_{\text {Perf }} \stackrel{\sim}{\longrightarrow} \operatorname{Sym}_{\mathcal{O}_{\text {Perf }}}(\mathcal{B})[2] \stackrel{\text { mult }}{\longrightarrow} \mathcal{B}[2] \simeq \mathcal{E}^{\vee} \otimes_{\mathcal{O}_{\text {Perf }}} \mathcal{E}[2] \stackrel{\operatorname{tr}[2]}{\longrightarrow} \mathcal{O}_{\text {Perf }}[2]
$$

where $\operatorname{tr}$ denotes the trace (or evaluation) map for the perfect complex $\mathcal{E}$. So, $\omega_{0}$ is a well defined, and non-degenerate 2-shifted 2-form on Perf. So, it only remains to show that there exists a 2-shifted closed 2-form $\omega$ on Perf, whose underlying 2-form is $\omega^{0}$. By [To-Ve-2, or Hoy, Theorem 2.1], one can prove that the weight 2 component of the refined Chern character $\mathrm{Ch}(\mathcal{E})$ (with values in negative cyclic homology) as defined in To-Ve-1 is indeed a 2-shifted closed 2-form on Perf whose underlying 2-form is $\frac{1}{2} \omega^{0}$, thus $\omega:=2 \mathrm{Ch}_{2}(\mathcal{E})$ is indeed a 2-shifted symplectic form on Perf whose underlying 2 -form is $\omega^{0}$.

By combining Theorem 1.2.1, 1.2.5 and 1.2.8, we get the following (non-exhaustive) list of geometrically interesting, classes of examples of $n$-shifted symplectic derived stacks:

- the derived stack Perf $(X):=\operatorname{MAP}_{\mathbf{d S t}}(X$, Perf $)$ of perfect complexes on a Calabi-Yau variety of dimension $d(n=$ $2-d)$.

- the derived stack $\operatorname{Perf}(M):=\mathrm{MAP}_{\mathrm{dSt}}(\operatorname{const}(M), \operatorname{Perf})$ of perfect complexes on a compact oriented topological manifold $M$ of dimension $d(n=2-d)$.

- the derived stack $\mathbb{R} \operatorname{Vect}_{n}(X)$ of rank $n$ vector bundles on a Calabi-Yau variety of dimension $d(n=2-d)$.

- the derived stack $\mathbb{R V e c t}{ }_{n}(M)$ of rank $n$ vector bundles on a compact oriented topological manifold $M$ of dimension $d(n=2-d)$.

- the derived stack $\mathbb{R B u n}_{G}(X)$ of $G$-torsors on a Calabi-Yau variety of dimension $d$, for $G$ reductive $(n=2-d)$.

- the derived stack $\mathbb{R} \operatorname{Loc}_{G}(M)$ of $G$-local systems on a compact oriented topological manifold $M$ of dimension $d$ $(n=2-d)$

- $\operatorname{MAP}_{\mathbf{d S t}}(X, Y)$, for $X$ a smooth and proper $d$-dimensional Calabi-Yau scheme, and $Y$ a smooth symplectic scheme $(n=-d)$.

- $\operatorname{MAP}_{\mathbf{d S t}}\left(X, \mathrm{~T}^{*} Y[m]\right)$, for $X$ a smooth and proper $d$-dimensional Calabi-Yau scheme, and $Y$ a derived Artin stack lfp over $k(n=m-d)$.

- iterated derived free loop spaces $\mathrm{MAP}_{\mathbf{d S t}}\left(\operatorname{const}\left(\left(S^{1}\right)^{d}\right), Y\right)$ of a smooth symplectic scheme $Y$, and more generally, $\mathrm{MAP}_{\mathrm{dSt}}(\operatorname{const}(M), Y)$, for $M$ a compact oriented topological $d$-manifold, and $Y$ a smooth symplectic scheme $(n=-d)$. - the derived stack $\mathbb{R} \operatorname{Loc}_{G}^{D R}(X)$ of flat $G$-bundles on $X$, a $d$-dimensional smooth and proper Deligne-Mumford stack $(n=2-2 d)$.

- the derived moduli stack $\mathrm{MAP}_{\mathrm{dSt}}\left(X_{\mathrm{Dol}}\right.$, Perf $)$ of Higgs fields on a proper and smooth Deligne-Mumford stack of dimension $d(n=2-2 d)$.

- the derived moduli stack $\mathcal{M}_{T}$ of compact objects in a $d$-Calabi-Yau dg-category $T$, e.g. the so-called non-commutative $K 3$ sub-dg-category of the derived dg-category of a cubic 4 -fold $(n=2-d$, and $d=2$ for a non-commutative $K 3) 4$

Remark 1.2.9 If $X$ is a proper and smooth scheme over $k$, and $D$ is a smooth Calabi-Yau divisor in the anti-canonical class, then Remark 1.2.7 together with Thms. 1.2.1 and 1.2.8 give us a lagrangian structure on the restriction map $\operatorname{Perf}(X)=\operatorname{MAP}_{\mathbf{d S t}}(X, \operatorname{Perf}) \rightarrow \operatorname{MAP}_{\mathbf{d S t}}(D, \operatorname{Perf})=\operatorname{Perf}(D)$, The same is true for the restriction map $\mathbb{R B u n} G(X) \rightarrow$ $\mathbb{R B u n}_{G}(D)$ between the derived stack of torsors under a smooth reductive group scheme $G$ over $k$.

As a sample consequence of Theorem [1.2.1, 1.2.5, and 1.2.8, we recall the following important result (BBJ, Corollary 5.19]) by Brav-Bussi-Joyce, establishing the existence of a local algebraic potential for Donaldson-Thomas moduli spaces attached to Calabi-Yau 3-folds.

Theorem 1.2.10 (Brav-Bussi-Joyce) The moduli space $\mathcal{M}_{D T}$ of simple coherent sheaves or of complexes of coherent sheaves on a Calabi-Yau 3-fold is Zariski-locally isomorphic, as a (-1)-shifted symplectic derived scheme, to the derived critical locus (as in Remark [1.2.6) of a regular function $f: U \rightarrow \mathbb{A}_{k}^{1}$ over a smooth $k$-scheme $U$.

\footnotetext{
${ }^{4}$ See e.g. [KS 3.3] for a definition of a $d$-Calabi-Yau dg-category, and [To2, 5.3] for a sketch of a proof of this result.
} 
The function in the statement is called the Donaldson-Thomas potential. Our existence theorems combine to give $\mathcal{M}_{D T}$ a (-1)-shifted symplectic structure, and the authors achieve Theorem 1.2.10 by proving a local structure theorem (Darboux style) for derived schemes $X$ endowed with a (-1)-shifted symplectic structure: any such $X$ is Zariski-locally a derived critical locus of a regular function on a smooth scheme. A similar statement in the $\mathbb{C}$-analytic category was proved before by Joyce-Song [JS, Theorem 5.4] using Gauge Theory, and a version valid formally locally at any point of $\mathcal{M}_{D T}$, by Kontsevich-Soibelman [KS, Section 3.3]. Obviously, Theorem 1.2.10 is a considerably stronger and more precise result.

\section{Shifted Poisson structures}

Setting up a notion of shifted Poisson structure for sufficiently general derived Artin stacks turns out to be much more complicated than the case of shifted symplectic structures, described in the previous section. On the other hand, a shifted Poisson structure on a derived stack $F$ is exactly the right structure that controls the deformation quantization of the $\infty$-category of perfect complexes on $F$. Therefore, in order to establish deformation quantizations for all the shifted symplectic derived moduli spaces listed at the end of Section 1 one also needs a comparison between shifted symplectic structures and (non-degenerate) shifted Poisson structures. Unfortunately, this comparison, which classically takes no more than two lines, is rather tricky in the derived setting, due to the fact that all the structures involved in the comparison are weak ones i.e. defined up-to-homotopy. The general theory of shifted Poisson structures, a comparison with shifted symplectic structures, and applications to deformation quantization of derived moduli spaces, have all been developed recently in [CPTVV], which is more than 100 pages long. In this Section we give a summary of the main constructions and results from [CPTVV], and a guide to its reading.

\subsection{Differential calculus}

In order to be able to define and study shifted Poisson structures on derived Artin stacks, we will need to have at our disposal a machinery of derived differential calculus (de Rham complex, shifted polyvectors etc.) working in sufficiently general setting. Thus, let $\mathcal{M}$ be a stable presentable symmetric monoidal $\infty$-category which is $k$-linear i.e. enriched over the $\infty$-category $\mathbf{d g}_{k}$ ([Lu4 , Definition 2.0.0.7]). In this paper, $\mathcal{M}$ will always be obtained as the coherent nerve of the Dwyer-Kan localization of a $\mathrm{dg}_{k}$-enriched symmetric monoidal model category $\mathrm{M}$ satisfying some additional technical properties for which we address the reader to CPTVV 1.1 and 1.2] 5 . And we will suppose that the enrichment is induced by a symmetric monoidal functor $\mathrm{dg}_{k} \rightarrow \mathrm{M}$.

For our present purposes, it will be enough to keep in mind some of the examples we will be most frequently interested in: $\mathcal{M}$ could be $\mathbf{d g}_{k}$ itself, the $\infty$-category $\mathbf{d g}_{k}^{g r}$ of $\mathbb{Z}$-graded dg-modules over $k$, the $\infty$-category $\epsilon-\mathbf{d g}_{k}$ of mixed dg-modules over $k$, the $\infty$-category $\epsilon-\mathbf{d g}_{k}^{g r}$ of $\mathbb{Z}$-graded mixed dg-modules over $k$, or more generally, any category of diagrams in the previous examples.

We denote by $\mathrm{CAlg}_{\mathbf{M}}$ the model category of commutative algebras in $\mathrm{M}$, and by $\left.\mathbf{C A} \lg _{\mathcal{M}}=L(\mathrm{CAlg})_{\mathrm{M}}\right)$, the corresponding $\infty$-category.

The symmetric monoidal model category $\epsilon-\mathrm{M}^{g r}$ of mixed graded objects in $\mathrm{M}$ is defined by replacing $\mathrm{dg}_{k}$ with $\mathrm{M}$ in the definition at the beginning of Section 1.1] (and with the cohomological shift defined here as $P[1]:=P^{k[-1]}$, for $P \in \mathrm{M}^{g r}$, using the co-tensor enrichment of $\mathrm{M}$ over $\mathrm{dg}_{k}$ ). The model category of commutative monoids in $\epsilon-\mathrm{M}^{g r}$ is denoted by $\epsilon-\mathrm{CAlg}_{\mathrm{M}}^{g r}$, and called the model category of graded mixed algebras in $\mathrm{M}$. The corresponding $\infty$ categories will be denoted by $\epsilon-\mathcal{M}^{g r}$, and $\epsilon-\mathbf{C A l g}_{\mathcal{M}}^{g r}$. Note that we have a canonical equivalence of $\infty$-categories $\mathbf{C A} \lg \left(\epsilon-\mathcal{M}^{g r}\right) \simeq \epsilon-\mathbf{C A l g}_{\mathcal{M}}^{g r}(\underline{\mathrm{Lu} 4}$, Theorem 4.5.4.7]).

\subsubsection{De Rham theory}

As explained in Remark1.1.5 for any $A \in \mathbf{C A l g}_{\mathcal{M}}$, we have the associated de Rham algebra $\mathbf{D R}^{\mathcal{M}}(A)$, a mixed graded algebra in $\mathcal{M}$, where $\mathbf{D R} \mathbf{R}^{\mathcal{M}}: \mathbf{C A} \lg _{\mathcal{M}} \rightarrow \epsilon-\mathbf{C A l g}_{\mathcal{M}}^{g r}$ is the left adjoint to the $\infty$-functor sending $B \in \epsilon-\mathbf{C A l g}_{\mathcal{M}}^{g r}$ to its weight 0 part $B(0)$. Note that if $A \in \mathbf{c d g a}_{k}$, then $\mathbf{D R}(A)$ of Definition 1.1.1 is exactly $\mathbf{D R} \mathbf{R}^{\mathcal{M}}(A)$, with $\mathcal{M}=\mathbf{d g}_{k}$. There is an $\infty$-functor

$$
A-\operatorname{Mod}_{\mathcal{M}} \longrightarrow \mathbf{T}, \quad P \longmapsto \operatorname{Der}(A, P):=\operatorname{Map}_{\mathbf{C A l g}_{\mathcal{M}} / A}(A, A \oplus P)
$$

\footnotetext{
${ }^{5} \mathrm{By}$ [Ni-Sa, it is likely that any $k$-linear stable presentable symmetric monoidal $\infty$-category can be obtained this way.
} 
which is co-represented by an $A$-module $\mathbb{L}_{A}^{\mathcal{M}}$ in $\mathcal{M}$, called the cotangent complex or cotangent object of $A$. As in Section [1.1, one can prove (CPTVV, Proposition 1.3.12]) that the canonical map

$$
\operatorname{Sym}_{A}\left(\mathbb{L}_{A}^{\mathcal{M}}[-1]\right) \longrightarrow \mathbf{D R}^{\mathcal{M}}(A)
$$

is functorial in $A$, and an equivalence in $\mathbf{C A l g} \mathbf{I g}_{\mathcal{M}}^{g r}$ (i.e. forgetting the mixed structure in the target). In other words, the construction $\mathbf{D} \mathbf{R}^{\mathcal{M}}$ endows the graded algebra $\operatorname{Sym} m_{A}\left(\mathbb{L}_{A}^{\mathcal{M}}[-1]\right.$ ) with a canonical (weak) mixed differential, the (weak) de Rham differential.

For the sake of brevity, we will omit the completely analogous definitions and results in the relative setting, i.e. for morphisms in $\mathbf{C A} \lg _{\mathcal{M}}$. We will instead say a few words about strict models of the above constructions, i.e. inside the model category M. First of all, if $A^{\prime} \in \mathbf{C A} \lg _{\mathbf{M}}$, then the functor

$$
A^{\prime}-\operatorname{Mod}_{\mathrm{M}} \longrightarrow \mathbf{T}, \quad P \longmapsto \operatorname{Der}\left(A^{\prime}, P\right):=\operatorname{Hom}_{\mathbf{C A l g}_{\mathrm{M}} / A^{\prime}}\left(A^{\prime}, A^{\prime} \oplus P\right)
$$

is co-represented by an $A^{\prime}$-module $\Omega_{\mathrm{M}, A^{\prime}}^{1}$ in $\mathrm{M}$ called the module of Kähler differentials of $A^{\prime}$. If $A \in \mathbf{C A} \lg _{\mathcal{M}}$ and $Q A$ is a cofibrant model for $A$ in $\mathbf{C A l g}_{\mathrm{M}}$, then we have a canonical equivalence $\Omega_{\mathrm{M}, Q A}^{1} \simeq \mathbb{L}_{A}^{\mathcal{M}}$ in $A-\mathbf{M o d}_{\mathcal{M}} \simeq Q A-\operatorname{Mod}_{\mathcal{M}}$. Furthermore, the functor $\epsilon-\mathbf{C A} \lg _{\mathrm{M}}^{g r} \rightarrow \mathbf{C A} \lg _{\mathrm{M}}$ selecting the weight 0 component, has a left adjoint $\mathbf{D} \mathbf{R}^{\mathrm{M}}$, thus for any $A^{\prime} \in \mathbf{C A} \lg _{\mathrm{M}}, \mathbf{D} \mathbf{R}^{\mathrm{M}}\left(A^{\prime}\right)$ is a strict mixed graded algebra in $\mathrm{M}$. Still for an arbitrary $A^{\prime} \in \mathbf{C} \mathbf{A} \lg _{\mathrm{M}}$, we also have a functorial isomorphism

$$
\operatorname{Sym}_{A^{\prime}}\left(\Omega_{\mathrm{M}, A^{\prime}}^{1}[-1]\right) \longrightarrow \mathbf{D R}^{\mathrm{M}}\left(A^{\prime}\right)
$$

in the category $\mathbf{C A l g} \mathbf{M}_{\mathbf{M}}^{g r}$ of strict graded algebras in M. This precisely says that $S y m_{A^{\prime}}\left(\Omega_{\mathrm{M}, A^{\prime}}^{1}[-1]\right)$ has a strict mixed structure, the de Rham differential. If $A \in \mathbf{C A} \lg _{\mathcal{M}}$ and $Q A$ is a cofibrant model for $A$ in $\mathbf{C A} \mathbf{l g}_{\mathrm{M}}$, then there is a an equivalence $\mathbf{D} \mathbf{R}^{\mathrm{M}}(Q A) \simeq \mathbf{D} \mathbf{R}^{\mathcal{M}}(A)$ in $\epsilon-\mathbf{C A} \lg _{\mathcal{M}}^{g r}$, i.e. $\mathbf{D} \mathbf{R}^{\mathrm{M}}(Q A)$, or equivalently $\operatorname{Sym}_{A^{\prime}}\left(\Omega_{\mathrm{M}, Q A}^{1}[-1]\right)$ with the induced mixed structure, is a strict model for $\mathbf{D} \mathbf{R}^{\mathcal{M}}(A)$ in $\epsilon-\mathbf{C A} \mathbf{l g}_{\mathcal{M}}^{g r}$.

Differential forms. Again as in Section 1.1, as suggested in Remark 1.1.5, we may define (closed) shifted differential forms for commutative algebras in $\mathcal{M}$.

Definition 2.1.1 Let $A \in \mathbf{C A l g}_{\mathcal{M}}$.

- The space of closed $n$-shifted $p$-forms on $A$ is

$$
\mathcal{A}_{\mathcal{M}}^{p, c l}(A, n):=\operatorname{Map}_{\epsilon-\mathcal{M}^{g r}}\left(\mathbf{1}_{\mathcal{M}}(p)[-p-n], \mathbf{D R}^{\mathcal{M}}(A)\right) \in \mathbb{T} .
$$

An element in $\pi_{0}\left(\mathcal{A}_{\mathcal{M}}^{p, c l}(A, n)\right)$ is called a closed $n$-shifted $p$-form on $A$.

- The space of $n$-shifted $p$-forms on $A$ is $\mathcal{A}_{\mathcal{M}}^{p}(A, n):=\operatorname{Map}_{\mathcal{M}}\left(\mathbf{1}_{\mathcal{M}}[-n], \wedge_{A}^{p} \mathbb{L}_{A}^{\mathcal{M}}\right) \in \mathbb{T}$. An element in $\pi_{0}\left(\mathcal{A}_{\mathcal{M}}^{p}(A, n)\right)$ is called a $n$-shifted $p$-form on $A$.

- Since $\operatorname{Sym}_{A}\left(\mathbb{L}_{A}^{\mathcal{M}}[-1]\right) \simeq \mathbf{D R}^{\mathcal{M}}(A)$, there is an induced map $u: \mathcal{A}^{p, c l}(A, n) \rightarrow \mathcal{A}^{p}(A, n)$, called the underlying $p$-form map.

If $\omega$ is a $n$-shifted 2-form on $A$, and we assume that $\mathbb{L}_{A}^{\mathcal{M}}$ is dualizable in $A-\operatorname{Mod}_{\mathcal{M}}$, then $u(\omega)$ induces a map $u(\omega)^{b}: \mathbb{T}_{A}^{\mathcal{M}} \rightarrow \mathbb{L}_{A}^{\mathcal{M}}[n]$ in $A-\operatorname{Mod}_{\mathcal{M}}$, where $\mathbb{T}_{A}^{\mathcal{M}}$ denotes the dual of $\mathbb{L}_{A}^{\mathcal{M}}$. We say that $\omega$ is non-degenerate if $u(\omega)^{b}$ is an equivalence.

Definition 2.1.2 Let $A \in \mathbf{C A l g}_{\mathcal{M}}$. The space of $n$-shifted symplectic structures on $A$ is the subspace $\operatorname{Sympl}(A, n)$ of $\mathcal{A}_{\mathcal{M}}^{p, c l}(A, n)$ whose connected components consist of non degenerate forms.

Remark 2.1.3 Note that, even if the notation $\operatorname{Sympl}(A, n)$ does not record $\mathcal{M}$, this space obviously depends on the category $\mathcal{M}$ inside which we are working. In Section 2.2, we will explain how the abstract Definition 2.1.2 gives back the definition of a shifted symplectic structure on a derived Artin stack (Definition 1.1.10). 


\subsubsection{Polyvectors}

Let $\mathbb{L} i e_{k}^{g r}$ be the graded Lie operad in $\mathrm{dg}_{k}$, where the bracket operation has degree -1 . We let dgLie ${ }_{k}^{g r}$ be the model category of $\mathbb{L} i e_{k}^{g r}$-algebras, i.e. graded Lie-algebras in $\mathrm{dg}_{k}$, where the (strict) Lie bracket has weight -1 ; we denote by $\mathbf{d g L i e}_{k}^{g r}$, the corresponding $\infty$-category $L\left(\mathrm{dgLie}_{k}^{g r}\right)$. By using our enriching functor $\mathrm{dg}_{k} \rightarrow \mathrm{M}$, we get an operad $\mathbb{L} i e_{\mathrm{M}}^{g r}$ in $\mathrm{M}$. Taking algebras with respect to this operad, we have $\mathrm{Lie}_{\mathrm{M}}^{g r}$, the model category of graded Lie-algebras in $\mathrm{M}$, and $\mathbf{L i e} \mathbf{M}_{\mathcal{M}}^{g r}$, the corresponding $\infty$-category $L\left(\operatorname{Lie}_{\mathrm{M}}^{g r}\right)$.

Similarly, for $n \in \mathbb{Z}$, we will denote by $\mathbb{P}_{n}$ the dg-operad (i.e. the operad in $\mathrm{dg}_{k}$ ) whose algebras are Poisson cdga's with a bracket of degree $(1-n)$. Recall that, for $n>1, \mathbb{P}_{n}$ can be identified with the operad of chains of the topological $n$-little disks operad $\mathbb{E}_{n}$ (see [Coh]). We will also be interested in a graded version of $\mathbb{P}_{n}$, denoted by $\mathbb{P}_{n}^{g r}$ : this is an operad in $\mathrm{dg}_{k}^{g r}$, whose algebras have multiplication of weight (= external grading) 0 , and bracket of weight -1 . The corresponding model categories of algebras will be denoted by $\mathbb{P}_{n}-\mathrm{Alg}_{\mathrm{dg}_{k}}=\mathbb{P}_{n}-\mathrm{cdga}_{k}$, and $\mathbb{P}_{n}^{g r}-\mathrm{Alg}_{\mathrm{dg}_{k}}=\mathbb{P}_{n}-\mathrm{cdga}_{k}^{g r}$; the associated $\infty$-categories by $\mathbb{P}_{n}-\mathbf{A l g}_{\mathrm{dg}_{k}}=\mathbb{P}_{n}-\mathbf{c d g a}_{k}$, and $\mathbb{P}_{n}^{g r}-\mathbf{A l g}_{\mathrm{dg}_{k}}=\mathbb{P}_{n}-\mathbf{c d g a}_{k}^{g r}$.

By using our enriching functor $\mathrm{dg}_{k} \rightarrow \mathrm{M}$, we thus get operads $\mathbb{P}_{\mathrm{M}, n}$ in $\mathrm{M}$, and $\mathbb{P}_{\mathrm{M}, n}^{g r}$ in $\mathrm{M}^{g r}$. Note that $\mathbb{P}_{\mathrm{M}, n}^{g r}$-algebras are commutative algebras $B$ in $\mathrm{M}^{g r}$ endowed with a bracket operation $[-,-]_{p, q}: B(p) \otimes_{\mathrm{M}} B(q) \rightarrow B(b+q-1)[1-n]$, which is a graded bi-derivation, and endows $B[n-1]$ with a Lie algebra structure inside $\mathrm{M}^{g r}$. The corresponding $\infty$-categories $L\left(\mathbb{P}_{\mathrm{M}, n}-\mathrm{Alg}\right)$ and $L\left(\mathbb{P}_{\mathrm{M}, n}^{g r}-\mathrm{Alg}\right)$ of algebras over these operads, will be denoted by $\mathbb{P}_{\mathcal{M}, n}-\mathbf{A l g}$ and $\mathbb{P}_{\mathcal{M}, n}^{g r}-\mathbf{A l g}$, respectively.

For $A^{\prime} \in \mathrm{CAlg}_{\mathrm{M}}, p \geq 0$, and $m \in \mathbb{Z}$, we define the object (in $\mathrm{M}$ ) of $m$-shifted, degree $p$ polyvectors $\operatorname{Pol}^{\mathrm{M}}\left(A^{\prime}, n\right)(p)$ on $A^{\prime}$, as follows. We consider $\mathrm{T}^{(p)}\left(A^{\prime}, m\right) \in \mathrm{M}$ the sub-object of $\underline{\operatorname{Hom}}_{\mathrm{M}}\left(A^{\prime \otimes^{p}}, A^{\prime}[m p]\right)$ (where $\underline{\operatorname{Hom}}_{\mathrm{M}}$ is the internal Hom-object in M) consisting of (shifted) multiderivations ([CPTVV, Section 1.4.2.]). There is a natural action of the symmetric group $\Sigma_{p}$ on $\underline{\operatorname{Hom}}_{\mathrm{M}}\left(A^{\prime \otimes^{p}}, A^{\prime}[m p]\right)$ induced by its standard action on $A^{\prime \otimes^{p}}$ and by the $(-1)^{m} \times \operatorname{Sign}$ action on $A^{\prime}[m p]$. this action restricts to an action on $\mathrm{T}^{(p)}\left(A^{\prime}, m\right)$, and we denote by $\mathrm{T}^{(p)}\left(A^{\prime}, m\right)^{\Sigma_{p}}$ the M-object of $\Sigma_{p}$-invariants. By standard conventions, we put $\mathrm{T}^{(0)}\left(A^{\prime}, m\right)=A^{\prime}$, and notice that $\mathrm{T}^{(1)}\left(A^{\prime}, m\right)=\underline{H o m}_{\mathrm{M}}\left(\Omega_{\mathrm{M}, A^{\prime}}^{1}, A^{\prime}[m]\right)$.

Definition 2.1.4 For $A^{\prime} \in \mathrm{CAlg}_{\mathrm{M}}$, and $n \in \mathbb{Z}$, we define the $\mathrm{M}^{g r}$-object of $n$-shifted polyvectors on $A^{\prime}$ to be

$$
\operatorname{Pol}^{\mathrm{M}}\left(A^{\prime}, n\right):=\bigoplus_{p} \mathrm{~T}^{(p)}\left(A^{\prime},-n\right)^{\Sigma_{p}}
$$

As in the classical case, there is a "composition" (by insertion) of shifted multiderivations and there is a SchoutenNijenhuis-like bracket of shifted multiderivations, so that $\operatorname{Pol}^{\mathrm{M}}\left(A^{\prime}, n\right)$ becomes a graded $\mathbb{P}_{n+1}$-algebra in $\mathrm{M}$, i.e. an object in $\mathbb{P}_{\mathrm{M}, n+1}-$ Alg.

Remark 2.1.5 If $A^{\prime}$ is such that $\Omega_{\mathrm{M}, A^{\prime}}^{1}$ is (strictly) dualizable in $A^{\prime}-\operatorname{Mod}_{\mathrm{M}}$, then it is easy to prove that there is an isomorphism $\mathrm{T}^{(p)}\left(A^{\prime}, m\right)^{\Sigma_{p}} \simeq \operatorname{Sym}^{p}\left(\mathrm{~T}_{\mathrm{M}, A^{\prime}}[-m]\right)$ in $\mathrm{M}$, and more generally $\operatorname{Pol}^{\mathrm{M}}\left(A^{\prime}, n\right) \simeq \oplus_{p \geq 0} \operatorname{Sym}^{p}\left(\mathrm{~T}_{\mathrm{M}, A^{\prime}}[-m]\right)$ in $\mathbb{P}_{\mathrm{M}, n+1}$ - Alg, where $\mathrm{T}_{\mathrm{M}, A^{\prime}}$ is the $A^{\prime}$-dual of $\Omega_{\mathrm{M}, A^{\prime}}^{1}$ (and, on the rhs the multiplication is the one induced by Sym, while the bracket is the one canonically induced by the Lie bracket in $\mathrm{T}_{\mathrm{M}, A^{\prime}}$. Note that, however, we have not used $\mathrm{T}_{\mathrm{M}, A^{\prime}}$ directly, in order to define Pol $^{\mathrm{M}}\left(A^{\prime}, n\right)$. We used multiderivations instead, and the the possible definitions agrees only under the hypotheses that $\Omega_{\mathrm{M}, A^{\prime}}^{1}$ is (strictly) dualizable.

The construction $A^{\prime} \mapsto \mathrm{Pol}^{\mathrm{M}}\left(A^{\prime}, n\right)$ is not fully functorial, since we can neither pullback nor pushforward multiderivations in general. However it is possible to define a restricted functoriality ([CPTVV, Lemma 1.4.13]) at the level of $\infty$-categories, enabling us to give the following

Definition 2.1.6 Let $n \in \mathbb{Z}$, and $\mathbf{C A l g} \lg _{\mathcal{M}}^{\text {fét }}$ the sub $\infty$-category of $\mathbf{C A} \lg _{\mathcal{M}}$ whose maps are only the formally étale ones (i.e. maps $A \rightarrow B$ such that $\mathbb{L}_{B / A} \simeq 0$ ): Then there is a well-defined $\infty$-functor

$$
\operatorname{Pol}^{\mathcal{M}}(-, n): \mathbf{C A l g}_{\mathcal{M}}^{\text {fét }} \longrightarrow \mathbb{P}_{\mathcal{M}, n+1}-\mathbf{A l g},
$$

such that if $A \in \mathbf{C A l g}_{\mathcal{M}}$ and $A^{\prime}$ is a fibrant-cofibrant replacement of $A$ inside $\mathrm{CAlg}_{\mathbf{M}}$, then we have an equivalence $\operatorname{Pol}^{\mathcal{M}}(A, n) \simeq \operatorname{Pol}^{\mathrm{M}}\left(A^{\prime}, n\right)$ in $\mathbb{P}_{\mathcal{M}, n+1}-\mathbf{A l g}$.

Remark 2.1.7 If $A \in \mathbf{C A l g}_{\mathcal{M}}$ is such that $\mathbb{L}_{A}^{\mathcal{M}}$ is dualizable in $A-\operatorname{Mod}_{\mathcal{M}}$, then one deduces from Remark 2.1.5 an equivalence $\operatorname{Pol}^{\mathcal{M}}(A, m) \simeq \oplus_{p \geq 0} \operatorname{Sym}^{p}\left(\mathbb{T}_{A}^{\mathcal{M}}[-m]\right)$. 
We are now in a position to give the definition of a shifted Poisson structure. Recall that if $A \in \mathbf{C A} \lg _{\mathcal{M}}$, then $\mathbf{P o l}^{\mathcal{M}}(A, n) \in \mathbb{P}_{\mathcal{M}, n+1}-\mathbf{A l g}$, so that, in particular, $\mathbf{P o l}^{\mathcal{M}}(A, n)[n] \in \mathbf{L} \mathbf{e}_{\mathcal{M}}^{g r}$.

Definition 2.1.8 Let $n \in \mathbb{Z}$, and $A \in \mathbf{C A l g}_{\mathcal{M}}$. The space of $n$-shifted Poisson structures on $A$ is

$$
\operatorname{Poiss}(A, n):=\operatorname{Map}_{\mathbf{L i e}}{ }_{\mathcal{M}}^{g r}\left(\mathbf{1}_{\mathcal{M}}[-1](2), \mathbf{P o l}^{\mathcal{M}}(A, n+1)[n+1]\right)
$$

where $\mathbf{1}_{\mathcal{M}}[-1](2)$ is the Lie algebra object in $\mathcal{M}$ given by $\mathbf{1}_{\mathcal{M}}$ sitting in pure cohomological degree 1 , and pure weight 2, with (strictly) trivial bracket.

This definition mimicks the classical one: if $\mathrm{M}=\mathrm{dg}_{k}$, and we replace Map by strict Hom-set in $\mathrm{dgLie}_{k}^{g r}$, in the above definition, we get exactly the set of Poisson bivectors on $A$ (the bracket being trivial on $\mathbf{1}[-1](2)=k[-1](2)$, the image of $1 \in k$ gives a biderivation on $\pi$ on $A$, such that $[\pi, \pi]=0)$.

\subsubsection{Standard realizations over $k$}

For the theory of shifted Poisson structures on derived stacks that we develop in Section 2.3 we will systematically need to pass to global sections. This procedure can be implemented already at the level of generality of the present section, by considering what we call standard realizations over $k$. A more technical notion of realization over $k$ (the Tate realization) will be discussed later on in this Section.

One of our standing hypotheses on the base symmetric monoidal $d g_{k}$ model category $M$ is that the unit $\mathbf{1}_{\mathbf{M}}$ is cofibrant. Hence we get a Quillen adjunction, with $\mathbf{1}_{\mathrm{M}} \otimes-$ left adjoint,

$$
\mathbf{1}_{\mathrm{M}} \otimes-: \mathrm{dg}_{k} \longleftrightarrow \mathrm{M}: \underline{\operatorname{Hom}}_{k}\left(\mathbf{1}_{\mathrm{M}},-\right)
$$

where $\otimes$ denotes the tensor $\mathrm{dg}_{k}$-enrichment of $\mathrm{M}$, and $\underline{H o m}_{k}$ is the Hom-enrichment of $\mathrm{M}$ in $\mathrm{dg}_{k}$. This induces a derived $\infty$-adjunction on the associated $\infty$-categories

$$
\mathbf{1}_{\mathrm{M}} \otimes-: \operatorname{dg}_{k} \longleftrightarrow \mathcal{M}: \mathbb{R} \underline{\operatorname{Hom}}_{k}\left(\mathbf{1}_{\mathrm{M}},-\right),
$$

and we define the standard realization functor as the right adjoint $|-|:=\mathbb{R} \underline{H o m}_{k}\left(\mathbf{1}_{\mathrm{M}},-\right)$. Actually this is the first of a series of realization functors induced on various categories of algebras in $\mathrm{M}$. In fact, since $\mathbf{1}_{\mathrm{M}}$ is a comonoid object in $\mathrm{M}$ (hence in $\mathcal{M}),|-|$ is actually a lax symmetric monoidal $\infty$-functor, hence it is a right adjoint on various functor on categories of "algebras" in $\mathcal{M}$. Our notation will always be $|-|$ for each of these induced realization functors. For example, we have

$|-|: \mathbf{C A l g}_{\mathcal{M}} \rightarrow \mathbf{c d g a}_{k}, \quad|-|: \mathbf{C A l g}_{\mathcal{M}}^{g r} \rightarrow \mathbf{c d g a}_{k}^{g r}, \quad|-|: \epsilon-\mathbf{C A l g} \operatorname{M}_{\mathcal{M}} \rightarrow \epsilon-\mathbf{c d g a}_{k}, \quad|-|: \epsilon-\mathbf{C A l g}_{\mathcal{M}}^{g r} \rightarrow \epsilon-\mathbf{c d g a}_{k}^{g r}$, $|-|: \mathbf{L i e}_{\mathcal{M}} \rightarrow$ dgLie $_{k}, \quad|-|: \mathbf{L i e}_{\mathcal{M}}^{g r} \rightarrow \operatorname{dgLie}_{k}^{g r}, \quad|-|: \mathbb{P}_{\mathcal{M}, n}-\mathbf{A l g} \rightarrow \mathbb{P}_{n}-$ cdga $_{k}, \quad|-|: \mathbb{P}_{\mathcal{M}, n}^{g r}-\mathbf{A l g} \rightarrow \mathbb{P}_{n}-$ cdga $_{k}^{g r}$. In particular we get realizations

$$
\left|\mathbb{L}_{B / A}^{\mathcal{M}}\right|=: \mathbb{L}_{B / A} \in \mathbf{d g}_{k}, \quad\left|\mathbf{D R}^{\mathcal{M}}(B / A)\right|=: \mathbf{D R}(B / A) \in \epsilon-\operatorname{cdga}_{k}^{g r}, \quad\left|\operatorname{Pol}^{\mathcal{M}}(A, n)\right|=: \operatorname{Pol}(A, n) \in \mathbb{P}_{n+1}-\mathbf{c d g a}_{k}^{g r} .
$$

Note that when $\mathcal{M}=\mathbf{d g}_{k}$, all this realization functor are (equivalent to) the identity functors.

Later on, we will consider realization functors on category of diagrams in $\mathcal{M}$, and it will be useful to recall the following fact. The $\mathbf{d g}_{k}$-enriched Hom in a category of diagrams $I^{o p} \rightarrow \mathcal{M}$, satisfies

$$
\underline{H o m}_{\mathcal{M}^{I^{o p}}}\left(\mathbf{1}_{\mathcal{M}^{I^{o p}}}, F\right) \simeq \lim _{x \in I^{o p}} \underline{H o m}_{\mathcal{M}}\left(\mathbf{1}_{\mathcal{M}}, F(x)\right),
$$

since the monoidal unit $\mathbf{1}_{\mathcal{M}^{I^{o p}}}$ is given by the constant $I^{o p}$-diagram at $\mathbf{1}_{\mathcal{M}}$. This observation is the reason for the appearance, in Thm 2.2.12 of (derived) global sections of various algebras over the derived affine site of a derived stack.

Example 2.1.9 As examples of the use of standard realization, by definition of $|-|$ as a right adjoint, we have, for any $A \in \mathbf{C A} \lg _{\mathcal{M}}$, the following equivalences in $\mathbf{T}$

$$
\begin{gathered}
\operatorname{Poiss}(A, n) \simeq \operatorname{Map}_{\mathbf{d g L i e}_{k}^{g r}}(k[-1](2), \operatorname{Pol}(A, n+1)[n+1]), \\
\mathcal{A}_{\mathcal{M}}^{p, c l}(A, n) \simeq \operatorname{Map}_{\epsilon-\mathbf{d g}_{k}^{g r}}(k(p)[-p-n], \mathbf{D R}(A)), \quad \mathcal{A}_{\mathcal{M}}^{p}(A, n) \simeq \operatorname{Map}_{\mathbf{d g}_{k}}\left(k[-n], \wedge_{|A|}^{p} \mathbb{L}_{A}\right) .
\end{gathered}
$$




\subsubsection{Comparison between Poisson algebras and shifted Poisson pairs}

We need a few notations. For an arbitrary $\infty$-category $\mathbf{C}, \mathcal{I}(\mathbf{C})$ will denote its moduli space or maximal $\infty$-subgroupoid of equivalences (so that $\mathcal{I}(\mathbf{C}) \in \mathbf{T}$ ), $\mathbf{C}^{\Delta[1]}$ the $\infty$-category of morphisms in $\mathbf{C}$, and $\mathrm{ev}_{0}, \mathrm{ev}_{1}: \mathbf{C}^{\Delta[1]} \rightarrow \mathbf{C}$ the source and target $\infty$-functors. For a model category $\mathrm{C}$, we denote by $\mathrm{C}_{c f}^{W}$ the category of cofibrant-fibrant objects in $\mathrm{C}$ with morphisms given by weak equivalences.

The nerve of $\mathrm{C}_{c f}^{W}$ is called the moduli space or classifying space (of objects and equivalences) of $\mathrm{C}$. It is a general fact (going back essentially to Dwyer and Kan) that we have an equivalence of spaces between the moduli space of C and the space $\mathcal{I}(\mathbf{C})$. In other words, we may (and will) identify the moduli space of $C$ with the moduli space of the associated $\infty$-category $\mathbf{C}$. We will be interested, below, in the moduli spaces of the $\infty$-categories $\mathbb{P}_{\mathcal{M}, n}-\mathbf{A l g}, \mathbf{L i e} \mathcal{M}_{\mathcal{M}}^{g r}$, and $\mathbf{C A l g} \lg _{\mathcal{M}}$, associated to the model categories $\mathbb{P}_{\mathrm{M}, n+1}-\mathrm{Alg}$, $\mathrm{Lie}_{\mathrm{M}}^{g r}$, and CAlg $\mathrm{M}_{\mathrm{M}}$, respectively.

For $A \in \mathbb{P}_{\mathcal{M}, n}-\mathbf{A l g}$, we will write $\mathbb{P}_{\mathcal{M}, n}(A)$ for the space of $\mathbb{P}_{n}$ algebra structures on $A$ having the given underlying commutative algebra structure. More precisely, there is a forgetful $\infty$-functor $U: \mathbb{P}_{\mathcal{M}, n}-\mathbf{A l g} \rightarrow \mathbf{C A} \lg _{\mathcal{M}}$ (forgetting the bracket structure), and the fiber of $U$ at a given $A \in \mathbf{C A l g}_{\mathcal{M}}$ is an $\infty$-groupoid, i.e. a space, that we denote by $\mathbb{P}_{\mathcal{M}, n}(A)$.

We have a canonical $\infty$-functor

$$
v: \mathcal{I}\left(\mathbf{C A l g} \operatorname{Mg}_{\mathcal{M}}\right) \ni A \longmapsto\left(\mathbf{1}[-1](2), \mathbf{P o l}^{\mathcal{M}}(A, n)[n]\right) \in \operatorname{LieAlg}_{\mathcal{M}}^{g r} \times \operatorname{LieAlg}_{\mathcal{M}}^{g r}
$$

(note that $A \mapsto \mathbf{P o l}^{\mathcal{M}}(A, n)$ is functorial with respect to equivalences in $\mathbf{C A l g}{ }_{\mathcal{M}}$, since obviously equivalences are formally étale). Borrowing ideas from [Vez2, 3.1], we define the space $\mathbf{P o i s s}_{\mathcal{M}, n}^{\mathrm{eq}}$ as the following pullback of in $\mathbf{T}$

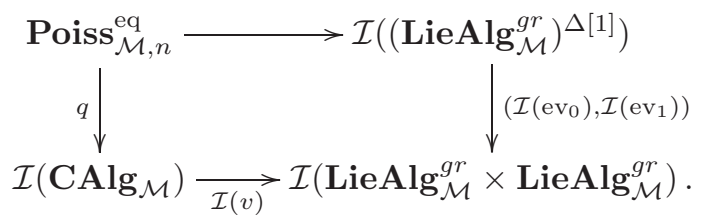

Thus, informally speaking, Poiss $\mathrm{eq}_{\mathcal{M}, n}$ is the moduli space of $n$-shifted Poisson pairs $(A, \pi)$, consisting of a commutative algebra $A$ in $\mathcal{M}$ together with a $n$-shifted Poisson structure on $A$ (see Definition 2.1.8). More precisely, let Poiss $_{\mathrm{M}, n}^{\mathrm{eq}}$ be the category whose objects are pairs $(A, \pi)$ where $A$ is a fibrant-cofibrant object in $\mathrm{CAlg}_{\mathrm{M}}$, and $\pi$ is a $n$-shifted Poisson structure on $A$, i.e a map $\mathbf{1}_{\mathrm{M}}[-1](2) \rightarrow \mathrm{Pol}^{\mathrm{M}}(A, n+1)[n+1]$ in the homotopy category of $\mathbf{L i e A} \mathbf{A g}_{\mathrm{M}}^{g r}$, and whose morphisms $(A, \pi) \rightarrow\left(A^{\prime}, \pi^{\prime}\right)$ are weak equivalences $u: A \rightarrow A^{\prime}$ in $\mathrm{CAlg}_{\mathrm{M}}$ such that the diagram

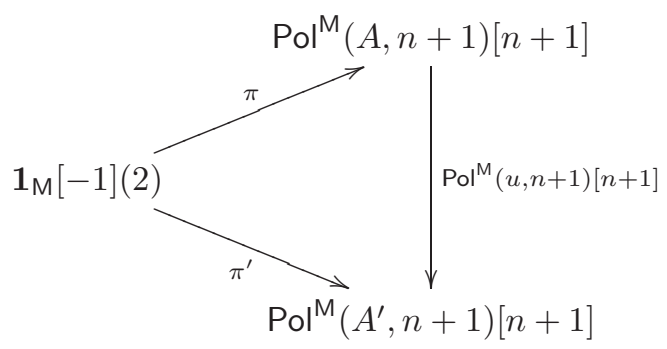

is commutative in the homotopy category of $\operatorname{LieAlg}_{\mathrm{M}}^{g r}$. Then, the nerve of Poiss $\mathrm{M}_{\mathrm{M}, n}^{\mathrm{eq}}$ is equivalent to Poiss $\mathrm{M}_{\mathcal{M}, n}^{\mathrm{eq}}$.

There is a (strict) functor $w: \mathbb{P}_{\mathrm{M}, n+1}-\mathbf{A l g}_{c f}^{W} \rightarrow$ Poiss $\mathrm{M}_{\mathrm{M}, n}^{\mathrm{eq}}$, sending a strict $\mathbb{P}_{n+1}$-algebra $B$ in $\mathrm{M}$ to the pair $(B, \pi)$, where $\pi$ is induced, in the standard way, by the (strict) Lie bracket on $B$ (since the bracket is strict, it is a strict biderivation on $B$ ). Restriction to weak equivalences (between cofibrant-fibrant objects) in $\mathbb{P}_{\mathrm{M}, n+1}-\mathbf{A l g}$ ensures this is a functor, and note that objects in the image of $w$ are, by definition, strict pairs $(B, \pi)$, i.e. the shifted Poisson structure $\pi: \mathbf{1}_{\mathrm{M}}[-1](2) \rightarrow \mathrm{Pol}^{\mathrm{M}}(A, n+1)[n+1]$ is an actual morphism in LieAlg $\lg _{\mathrm{M}}^{g r}$ (rather than a map in its homotopy category). We have a commutative diagram of functors

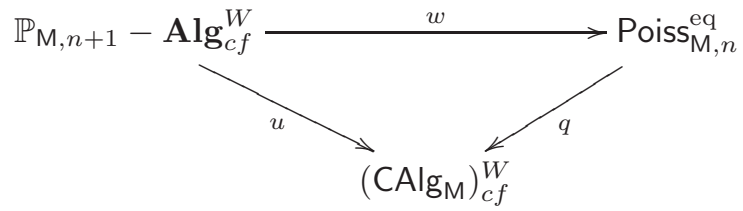


where $u$ forgets the bracket structure, and $q$ is the functor $(A, \pi) \mapsto A$. Taking the nerves of the previous diagram, we get a commutative diagram in $\mathbf{T}$ (where we have kept the same name for the maps)

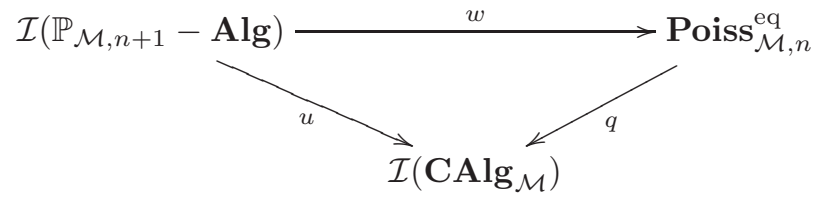

Note that $u$, and $q$ are both surjective, since they both have a section given by the trivial bracket or strict shifted Poisson structure. Moreover, the fiber of $q$ at $A \in \mathbf{C A l g}_{\mathcal{M}}$ is obviously equivalent to the space $\operatorname{Poiss}_{\mathcal{M}}(A, n)$ of $n$-shifted Poisson structures on $A$ (Definition 2.1.8). The following is a slight enhancement of [Me, Theorem 3.2].

Theorem 2.1.10 The map of spaces $w: \mathcal{I}\left(\mathbb{P}_{\mathcal{M}, n+1}-\mathbf{A l g}\right) \rightarrow$ Poiss $_{\mathcal{M}, n}^{\mathrm{eq}}$ is an equivalence.

Proof. It is enough to prove that for any fibrant-cofibrant $A \in \mathrm{CAlg}_{\mathrm{M}}$, the map induced by $w$ between the $u$ and $q$ fibers over $A$ is an equivalence. But, since these fibers are, by definition, $\mathbb{P}_{\mathcal{M}, n+1}(A)$ (the moduli space of $\mathbb{P}_{n}$-algebra structures on $A$ having the given underlying commutative algebra structure), and Poiss $\mathcal{M}(A, n)$ (the space of $n$-shifted Poisson structure on $A$ ), this is exactly [Me, Theorem 3.2].

For future reference, we also state the following immediate consequence.

Corollary 2.1.11 (Melani) For any $A \in \mathbf{C A l g}_{\mathcal{M}}$, the map $w$ of Theorem 2.1.10 induces an equivalence $w_{A}: \mathbb{P}_{\mathcal{M}, n+1}(A) \simeq \operatorname{Poiss}_{\mathcal{M}}(A, n)$ between the moduli space of $\mathbb{P}_{n}$-algebra structures on $A$ (having the given underlying commutative algebra structure), and the moduli space of $n$-shifted Poisson structures on $A$.

Proof. As mentioned above, this is exactly [Me, Theorem 3.2].

Remark 2.1.12 (1) Theorem 3.2 in $[\mathrm{Me}$ is stated for the model category $\mathrm{M}$ of bounded above cochain complexes of $k$-modules, but the proof is general and it extends immediately to our general $\mathrm{M}$. The original statement seems moreover to require a restriction to those cdga's having a dualizable cotangent complex. This is due to the fact that the author uses the tangent complex (i.e. the dual of the cotangent complex) in order to identify derivations. However, the actual proof produces an equivalence between (weak, shifted) Lie brackets and (weak) biderivations. Therefore if one identifies derivations using the linear dual of the symmetric algebra of the cotangent complex, the need to pass to the tangent complex disappears, and the result holds, with the same proof and without the assumption of the cotangent complex being dualizable (see Remark 2.1.7). This is the main reason we adopted Definition 2.1.4 and 2.1.6 as our definition of polyvectors.

(2) Melani's proof of $\left[\mathrm{Me}\right.$, Theorem 3.2] also shows that the natural map from the classifying space $\mathcal{I}\left(\mathbb{P}_{\mathcal{M}, n+1}-\mathbf{A l g}\right)$ of strict $\mathbb{P}_{n+1}$-algebras in $\mathrm{M}$ to the classifying space $\mathcal{I}\left(\mathbb{P}_{\mathcal{M}, n+1, \infty}-\mathbf{A l g}\right)$ of weak $\mathbb{P}_{n+1}$-algebras in $\mathrm{M}$ (where the operad $\mathbb{P}_{\mathcal{M}, n+1, \infty}$ is any cofibrant resolution of $\mathbb{P}_{\mathcal{M}, n+1}$ in the model category of operads in $\left.\mathcal{M}\right)$ is an equivalence.

We also have the following, easier, strict analog of Corollary 2.1.11

Proposition 2.1.13 [CPTVV, Proposition 1.4.8] For any $A \in \mathrm{CAlg}_{\mathrm{M}}$, there is a natural bijection

$$
\mathbb{P}_{\mathrm{M}, n}(A) \simeq \operatorname{Hom}_{L i e_{\mathrm{M}}^{g r}}\left(\mathbf{1}_{\mathrm{M}}(2)[-1], \mathrm{Pol}^{\mathrm{M}}(A, n)[n]\right)
$$

between the set of (strict) $\mathbb{P}_{n}$-algebra structures on $A$ in $\mathrm{M}$, and the set of morphisms $1(2)[-1] \rightarrow \operatorname{Pol}^{\mathrm{M}}(A, n)[n]$ of Lie algebra objects in $\mathrm{M}^{g r}$.

As an immediate consequence of Theorem 2.1.10, we get the following, useful, strictification result

Corollary 2.1.14 Any weak shifted Poisson pair in Poiss $\mathrm{M}, n^{e q}$ is equivalent to a strict pair. 
Proof. By Theorem 2.1.10, an object $(A, \pi) \in \operatorname{Poiss}_{\mathrm{M}, n}^{\mathrm{eq}}$ (i.e. an a priori weak pair), is equivalent to a pair of the form $w(B)$, where $B \in \mathbb{P}_{\mathrm{M}, n+1}-\mathbf{A l g}$ (i.e. is a strict $\mathbb{P}_{n+1}$-algebra in $\mathbf{M}$ ), whose underlying commutative algebra is weakly equivalent to $A$ in $\mathrm{CAlg}_{\mathrm{M}}$. We conclude by observing that objects in the image of $w$ are always strict pairs.

The DR-to-Pol construction. Let $A^{\prime} \in \mathbb{P}_{\mathrm{M}, n}-\mathbf{A l g}$. By Proposition 2.1.13, the $\mathbb{P}_{n}$ algebra structure on the underlying commutative algebra of $A^{\prime}$ is encoded by a strict map $\pi: 1_{\mathrm{M}}[-1](2) \rightarrow \mathrm{Pol}^{\mathrm{M}}\left(A^{\prime}, n\right)[n]$ of graded Lie algebras in $\mathrm{M}$. Since the weight $q$ part of $\operatorname{Pol}^{\mathrm{M}}\left(A^{\prime}, n\right)$ is, by definition, $\mathrm{T}^{(q)}\left(A^{\prime},-n\right)^{\Sigma_{q}}, \pi$ induces a map $\mathbf{1}_{M} \rightarrow \mathrm{T}^{(2)}\left(A^{\prime},-n\right)^{\Sigma_{2}}[n+1]$ that we still denote by $\pi$. Write

$$
[-,-]_{p, q}: \operatorname{Pol}^{\mathrm{M}}\left(A^{\prime}, n\right)(p) \otimes_{\mathrm{M}} \operatorname{Pol}^{\mathrm{M}}\left(A^{\prime}, n\right)(q) \rightarrow \operatorname{Pol}^{\mathrm{M}}\left(A^{\prime}, n\right)(p+q-1)[-n]
$$

for the Lie bracket part of the graded $\mathbb{P}_{n+1}$-algebra structure on $\mathrm{Pol}^{\mathrm{M}}\left(A^{\prime}, n\right)$, the family of composite maps in $\mathrm{M}$

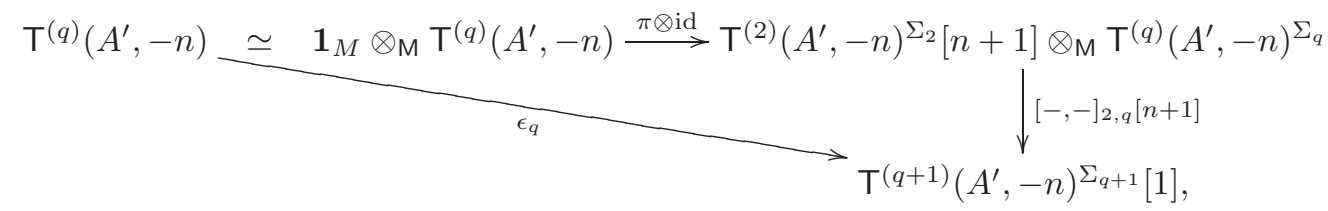

for $q \in \mathbb{N}$, is easily verified to be the mixed differential of a mixed graded algebra structure on $\mathrm{Pol}^{\mathrm{M}}\left(A^{\prime}, n\right)$ inside $\mathrm{M}$. Moreover, since $\mathrm{Pol}^{\mathrm{M}}\left(A^{\prime}, n\right)(0)=A^{\prime}$, by the universal property of $\mathrm{DR}^{\mathrm{M}}\left(A^{\prime}\right)$ the identity $A^{\prime} \rightarrow A^{\prime}$ induces a map

$$
\phi_{A^{\prime}, \pi}: \mathrm{DR}^{\mathrm{M}}\left(A^{\prime}\right) \longrightarrow \operatorname{Pol}^{\mathrm{M}}\left(A^{\prime}, n\right)
$$

of mixed graded algebras in $\mathrm{M}$.

Remark 2.1.15 The above construction of the mixed differential $\epsilon=\left(\epsilon_{q}\right)_{q}$ is a generalization of the classical construction associating to a classical Poisson bivector $\pi$ on a smooth manifold, the differential $d_{\pi}:=[\pi,-]$ on polyvectors, where $[-,-]$ is the Schouten-Nijenhuis bracket, and $d_{\pi}^{2}=0$ is equivalent to the bivector $\pi$ being Poisson.

A slight elaboration of this construction (by choosing strict models, see [CPTVV, 1.4.3] for details), yields the following derived version

- functors $\mathbf{D R}{ }^{\mathcal{M}}, \mathbf{P o l}^{\mathcal{M}}(-, n):\left(\mathbb{P}_{\mathcal{M}, n+1}-\mathbf{A l g}\right)^{e q} \rightarrow \epsilon-\mathbf{C A l g}_{\mathcal{M}}^{g r}$

- a morphism $\Phi: \mathbf{D} \mathbf{R}^{\mathcal{M}} \rightarrow \mathbf{P o l}^{\mathcal{M}}(-, n)$ betwen the above functors.

Here $\left(\mathbb{P}_{\mathcal{M}, n+1}-\mathbf{A l g}\right)^{e q}$ is the $\infty$-category of $\mathbb{P}_{n+1}$-algebras in $\mathcal{M}$ with only equivalences as morphisms (this ensures that $\mathbf{P o l}^{\mathcal{M}}(-, n)$ is indeed a functor), and we slightly abuse notation by writing $\mathbf{D} \mathbf{R}^{\mathcal{M}}$ for the composition of the usual $\mathbf{D R}^{\mathcal{M}}$ with the forgetful functor $\left(\mathbb{P}_{\mathcal{M}, n+1}-\mathbf{A l g}\right)^{e q} \rightarrow \mathbf{C A l g}_{\mathcal{M}}$.

\subsubsection{From non-degenerate Poisson algebra structures to symplectic structures}

Classically, one way of stating that a Poisson structure (on a smooth scheme or manifold $X$ ) is non degenerate is by declaring that the analog of the above map $\Phi$ establishes an isomorphism of mixed graded algebras between the de Rham algebra and the algebra of polyvectors. Analogously, we say that $A \in \mathbb{P}_{\mathcal{M}, n+1}-\mathbf{A l g}$ is non degenerate if $\Phi_{A}: \mathbf{D} \mathbf{R}^{\mathcal{M}}(A) \rightarrow \mathbf{P o l}^{\mathcal{M}}(A, n)$ is an equivalence of mixed graded algebras in $\mathcal{M}$. For such an $A$, by Corollary 2.1.11. we also get a map $\alpha_{A}: \mathbf{1}_{\mathcal{M}}(2) \rightarrow \mathbf{P o l}^{\mathcal{M}}(A, n)[n+1]$ in $\epsilon-\mathbf{M}^{g r}$. By putting these together, we get a diagram

$$
\mathbf{D R}^{\mathcal{M}}(A)[n+1] \stackrel{\Phi_{A}}{\longrightarrow} \mathbf{P o l}^{\mathcal{M}}(A, n)[n+1] \stackrel{\alpha_{A}}{\longleftarrow} \mathbf{1}_{\mathcal{M}}(2)
$$

in $\epsilon-\mathbf{M}^{g r}$, exhibiting both $\mathbf{D} \mathbf{R}^{\mathcal{M}}(A)[n+1]$ and $\mathbf{1}_{\mathcal{M}}(2)$ as objects in the overcategory $\epsilon-\mathbf{M}^{g r} / \mathbf{P o l}^{\mathcal{M}}(A, n)[n+1]$. We can then give the following

Definition 2.1.16 Let $A \in \mathbb{P}_{\mathrm{M}, n+1}-\mathbf{A l g}$. The space of closed 2-shifted forms compatible with the given $\mathbb{P}_{n}$-structure on $A$ is the space of lifts of $\alpha_{A}$ along $\Phi_{A}$, i.e. the space

$$
\operatorname{Comp}_{\mathcal{M}}^{2, c l}(A, n):=\operatorname{Map}_{\epsilon-\mathbf{M}^{g r} / \mathbf{P o l}^{\mathcal{M}}(A, n)[n+1]}\left(\mathbf{1}_{\mathcal{M}}(2), \mathbf{D R}^{\mathcal{M}}(A)[n+1]\right) .
$$


The reasons for using the words "closed forms" in the previous definition are the following. First of all there is a "forgetful" map

$$
\operatorname{Comp}_{\mathcal{M}}^{2, c l}(A, n) \longrightarrow \operatorname{Map}_{\epsilon-\mathbf{M}^{g r}}\left(\mathbf{1}_{\mathcal{M}}(2), \mathbf{D R}^{\mathcal{M}}(A)[n+1]\right)=\mathcal{A}_{\mathcal{M}}^{2, c l}(A, n-1)
$$

to the actual space of closed 2-shifted forms on $A$. Moreover, if $A$ happens to be non degenerate, then $\operatorname{Comp}_{\mathcal{M}}^{2, c l}(A, n-1)$ is contractible (since $\Phi_{A}$ is an equivalence), hence there is a unique closed $(n-1)$-shifted 2-form $\omega_{A} \in \pi_{0}\left(\mathcal{A}_{\mathcal{M}}^{2, c l}(A, n-1)\right)$ on $A$, via the above "forgetful" map. Moreover, by definition, such an $\omega_{A}$ is non degenerate since $A$ is: $\omega$ is thus a symplectic structure on $A$. If we denote by $\mathbb{P}_{\mathcal{M}, n}^{n d}(A)$ the subspace of $\mathbb{P}_{\mathcal{M}, n}(A)$ whose connected components consist of non degenerate elements, we get the following

Proposition 2.1.17 For $A \in \mathrm{CAlg}_{\mathcal{M}}$ the above construction yields a well-defined map of spaces $W_{A}: \mathbb{P}_{\mathcal{M}, n}^{n d}(A) \rightarrow \operatorname{Sympl}_{\mathcal{M}}(A, n-1)$.

There is a parallel (and in fact equivalent) notion of non-degenerate shifted Poisson structure in $\mathcal{M}$. Let $B \in \mathbf{C A l g}$ such that $\mathbb{L}_{B}^{\mathcal{M}}$ is a dualizable in $B-\operatorname{Mod}_{\mathcal{M}}$. An $n$-shifted Poisson structure $\pi \in \pi_{0}\left(\operatorname{Poiss}_{\mathcal{M}}(B, n)\right)$ defines a map $\mathbf{1}_{\mathcal{M}} \rightarrow \operatorname{Sym}_{B}^{2}\left(\mathbb{T}_{B}^{\mathcal{M}}[-n-1]\right)[n+2]$ in $\mathcal{M}$, and thus induces, by adjunction, a map $\mathbb{L}_{B}^{\mathcal{M}} \rightarrow \mathbb{T}_{B}^{\mathcal{M}}[-n]$ in $B-\operatorname{Mod}_{\mathcal{M}}$ : we say that $\pi$ is non degenerate if this map is an equivalence. The subspace of $\operatorname{Poiss}_{\mathcal{M}}(B, n)$ whose connected components consist of non degenerate elements will be denoted by $\operatorname{Poiss}_{\mathcal{M}}^{n d}(B, n)$. The notions of non degeneracy for Poisson algebras and for Poisson structures coincide in the following sense

Proposition 2.1.18 Let $B \in \mathbf{C A l g}_{\mathcal{M}}$ such that $\mathbb{L}_{B}^{\mathcal{M}}$ is a dualizable in $B-\operatorname{Mod}_{\mathcal{M}}$. The equivalence $w_{B}$ of Corollary 2.1.11, restricts to an equivalence $\mathbb{P}_{\mathcal{M}, n}^{n d}(B) \simeq \operatorname{Poiss}_{\mathcal{M}}^{n d}(B, n-1)$. As a consequence of Proposition 2.1.17, we thus get a map

$$
W_{B}^{\prime}: \operatorname{Poiss}_{\mathcal{M}}^{n d}(B, n-1) \longrightarrow \operatorname{Sympl}_{\mathcal{M}}(B, n-1)
$$

The map $W_{B}^{\prime}$ in Proposition 2.1.18 is called the comparison map between non degenerate shifted Poisson structures and shifted symplectic structures. We will study it for derived Artin stacks in Section 2.4.

Remark 2.1.19 It is possible that the map $W_{B}^{\prime}$ in Proposition 2.1.18 will turn out to be an equivalence, for very general $\mathcal{M}$. We have proven this in our geometric case of interest (see Theorem 2.4.2). The difficulty in general stems from the fact that while for the source of $W_{B}^{\prime}$ we have Theorem 2.1.11 and Proposition 2.1.13, and we thus are able to perform the DR-to-Pol construction and produce the map $W_{B}^{\prime}$, we don't have anything similar for the target of $W_{B}^{\prime}$. One runs into serious difficulties even just trying to construct an inverse equivalence to $W_{B}^{\prime}$ at the level of connected components. The first obstacle is that a shifted symplectic structure is, by definition, a weak map (i.e. a map in the relevant homotopy category). Even if we could strictify this map (getting a strictly closed shifted 2-form), we are still left with the problem that non degeneracy is a weak property, i.e. the property of a map being a quasi-isomorphism, and therefore cannot be readily used to build a strict Lie bracket on the de Rham algebra. One might be able to overcome these difficulties in general but we do not know how to do this at the moment.

\subsubsection{Tate realizations over $k$}

In this section, we will concentrate on the special case $\mathrm{M}=\epsilon-\mathrm{dg}_{k}^{g r}$, with its associated $\infty$-category $\mathcal{M}=\epsilon-\mathbf{d g}^{g r}$, and in the next Section we will apply the definitions and results obtained here to categories of diagrams in $\mathcal{M}$.

The unit of the symmetric monoidal category $\mathrm{M}$ is $k(0)$, i.e. the complex $k[0]$ sitting in pure weight 0 , with the trivial mixed differential. The enriching symmetric monoidal functor is given by $\operatorname{dg}_{k} \rightarrow \mathrm{M}: V \mapsto V(0)$, the tensor enrichment is then given by $V \otimes E:=V(0) \otimes_{\mathrm{M}} E$, for $E \in \mathrm{M}$. The enriched hom object is thus

$$
\underline{\operatorname{Hom}}_{k}\left(E, E^{\prime}\right):=\mathrm{Z}_{\epsilon}\left(\underline{H o m}_{\mathcal{M}}\left(E, E^{\prime}\right)(0)\right) \in \mathrm{dg}_{k},
$$

where $\underline{H o m}_{M}$ denotes the internal Hom in $\mathrm{M}$, and, for $F \in \mathrm{M}$, we write $\mathrm{Z}_{\epsilon}(F(0)) \in \mathbf{d g}_{k}$ for the kernel of the map of $k$-dg-modules $\epsilon: F(0) \rightarrow F(1)[1]$.

The standard realization functor $|-|: \mathcal{M} \rightarrow \mathbf{d g}_{k}$, for $\mathcal{M}=\epsilon-\mathbf{d g}^{g r}$, is in some sense unsatisfactory since an easy computation ( CPTVV Proposition 1.5.1]) shows that $|E| \simeq \prod_{p \geq 0} E(p)$, for $E \in \mathcal{M}$, so that all negative weights are lost under standard realization. An obvious way to modify $T-\mid$ and remedy this flaw is to consider $|E|^{t}:=\operatorname{colim}_{i \geq 0} \prod_{p \geq-i} E(p)$, instead. This new functor $|-|^{t}: \mathcal{M} \rightarrow \mathbf{d g}_{k}$ will be called the Tate realization functor 
for $\mathcal{M}$. By definition, there is a canonical morphism $|-| \rightarrow|-|^{t}$ of $\infty$-functors. One can show, exactly as for $|-|$, that $|-|^{t}$ is lax symmetric monoidal as well, so that it is inherited by categories of algebras. In particular, we also get Tate realization functors

$$
|-|^{t}: \epsilon-\mathbf{C A l g}_{\mathcal{M}}^{g r} \rightarrow \epsilon-\mathbf{c d g a}_{k}^{g r}, \quad|-|^{t}: \mathbb{P}_{\mathcal{M}, n}^{g r}-\mathbf{A l g} \rightarrow \mathbb{P}_{n}-\mathbf{c d g a}_{k}^{g r}
$$

As in the linear case, there are canonical morphisms $|-| \rightarrow|-|^{t}$ of $\infty$-functors between realizations on the level of these algebra structures.

Let us put this into a broader perspective, and relate the Tate realization to a standard realization (on a different category). Let us start by the fact that there is an equivalence in $\mathbf{d g}_{k}$

$$
\underline{\mathcal{H o m}}_{k}(k(i), k(i+1))=\underline{\mathbb{H o m}}_{k}(k(i), k(i+1)) \simeq k[0]
$$

(where $\underline{\mathcal{H} o m}_{k}$ denotes the $\mathbf{d g}_{k}$-enriched Hom in $\mathcal{M}$ ), giving rise to the following canonical ind-object in $\mathcal{M}$

$$
k(\infty):=\{k(0) \rightarrow k(1) \rightarrow \cdots \rightarrow k(i) \rightarrow k(i+1) \rightarrow \cdots\} \in \operatorname{Ind}(\mathcal{M})
$$

One can then show that the Tate realization functor for $\mathcal{M}$ is related to the standard realization $|-|$ Ind for Ind $(\mathcal{M})$, by

$$
|-|^{t} \simeq|-\otimes k(\infty)|_{\text {Ind }}: \mathcal{M} \longrightarrow \mathbf{d g}_{k}
$$

where we have implicitly used the canonical functor $\mathcal{M} \rightarrow \operatorname{Ind}(\mathcal{M})$, sending an object $E$ to the constant ind-diagram in $\mathcal{M}$ with value $E$.

Moreover, since $k(i) \otimes k(j) \simeq k(i+j), k(\infty)$ is a commutative monoid object in $\operatorname{Ind}(\mathcal{M})$, hence

$$
A \in \mathbf{C A l g}_{\mathcal{M}} \Rightarrow A(\infty):=A \otimes k(\infty) \in k(\infty) / \mathbf{C A} \lg _{\operatorname{Ind}(\mathcal{M})}
$$

Therefore it make sense to consider the relative objects

$$
\mathbf{D R}^{\operatorname{Ind}(\mathcal{M})}(A(\infty) / k(\infty)) \in \epsilon-\mathbf{A l g}_{\mathbf{I n d}(\mathcal{M})}^{g r}, \quad \operatorname{Pol}^{\operatorname{Ind}(\mathcal{M})}(A(\infty) / k(\infty), n) \in \mathbb{P}_{\mathbf{I n d}(\mathcal{M}), n+1}-\mathbf{A l g}^{g r},
$$

and their standard realizations (on algebras in $\operatorname{Ind}(\mathcal{M})$ )

$$
\operatorname{DR}(A(\infty) / k(\infty)) \in \epsilon-\mathbf{c d g a}_{k}^{g r}, \quad \operatorname{Pol}(A(\infty) / k(\infty), n) \in \mathbb{P}_{n+1}-\mathbf{c d g a}_{k}^{g r}
$$

For de Rham algebras and polyvectors, we have the following comparison result

Proposition 2.1.20 If $A \in \mathbf{C A l g}_{\mathcal{M}}$, then we have a canonical equivalence

$$
\mathbf{D R}^{t}(A):=|\mathbf{D R}(A)|^{t} \simeq \mathbf{D R}(A(\infty) / k(\infty))
$$

in $\epsilon-\mathbf{c d g a}_{k}^{g r}$. If moreover $\mathbb{L}_{A}^{\mathcal{M}}$ is dualizable in $A-\operatorname{Mod}_{\mathcal{M}}$, then we have a canonical equivalence

$$
\operatorname{Pol}^{t}(A, n):=|\operatorname{Pol}(A, n)|^{t} \simeq \operatorname{Pol}(A(\infty) / k(\infty))
$$

in $\mathbb{P}_{n+1}-\operatorname{cdga}_{k}^{g r}$.

Remark 2.1.21 As already observed in the linear case, in general, none of the morphisms $\mathbf{D R}(A) \rightarrow \mathbf{D R}^{t}(A)$, $\mathbf{P o l}(A, n) \rightarrow \mathbf{P o l}^{t}(A, n)$ are equivalences. If $A \in \epsilon-\mathbf{C A} \mathbf{l g}_{\mathcal{M}}^{g r}$ happens to have only non-negative weights (this will be the case in our application to derived stacks), then also $\mathbb{L}_{A}$ will have the same property, and $\mathbf{D R}(A) \rightarrow \mathbf{D R} \mathbf{R}^{t}(A)$ will indeed

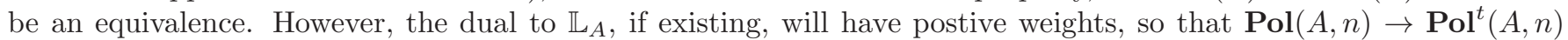
will not be an equivalence, even in this case. So, at least for applications to derived stacks, while the introduction of the Tate realization will not make any difference for DR, it will definitely do for Pol, and indeed the interesting realization will be $\mathbf{P o l}^{t}$ rather than the standard one. 


\subsection{Formal derived stacks and formal localization}

A crucial ingredient in the theory of shifted Poisson structures on general derived Artin stacks is the method of formal localization. Formal localization is interesting in its own right as a new, very powerful tool that will prove useful in many other situations inside derived geometry, especially in order to globalize tricky constructions and results, whose extension from the local case presents obstructions that only vanish formally locally. An example is given by obstructions living in de Rham cohomology (even, say, on a smooth scheme $X$ ). Suppose we wish to glue some construction that we can perform "locally" on $X$, and we know that obstructions to globalize live in de Rham cohomology of $X$ (e.g. we would like to globalize an algebraic version of the Darboux lemma). Since de Rham cohomology never vanishes Zariski or étale locally, it is going to be hard or impossible, depending on the specific problem, to suppress the obstructions and glue with respect to these topologies. On the other hand, for any $x \in X$, the de Rham cohomology of the formal completion $\widehat{X_{x}}$ does vanish, so we may try to glue the construction performed on the family $\left\{\widehat{X_{x}} \mid x \in X\right\}$ to a construction on $X$. But in order to do this we need a result telling us when and how we are able to glue objects along the family of formal completions. This is exactly the content of formal localization and below we will sketch how it works and why it is useful.

The following conventions will be adopted throughout this section. A cdga $A$ is called almost finitely presented if $H^{0}(A)$ is a $k$-algebra of finite type, and each $H^{i}(A)$ is a finitely presented $H^{0}(A)$-module. We will write $\mathbf{d A f f}{ }_{k}$ for the opposite $\infty$-category of almost finitely presented cdga's, and we will simply refer to its objects as derived affine schemes without mentioning the finite presentation condition. In particular, when writing $\operatorname{Spec} A$, we implicitly assume that Spec $A$ is an object of $\mathbf{d} \mathbf{A f f}{ }_{k}$. The $\infty$-category $\mathbf{d A f f}{ }_{k}$ is equipped with its usual étale topology of [HAG-II, Definition 2.2.2.3] , and the corresponding $\infty$-topos of derived stacks will be denoted by $\mathbf{d S t}_{k}$. Its objects will simply be called derived stacks, instead of the more precise but longer locally almost finitely presented derived stacks over $k$. With these conventions, an algebraic derived $n$-stack $X$ will have a smooth atlas by objects in $\mathbf{d A f f}{ }_{k}$, and in particular, it will have a bounded above cotangent complex in $\operatorname{Coh}(X)$.

\subsubsection{Formal derived stacks}

As the name suggests, formal localization deals with formal derived stacks, which we now define.

Definition 2.2.1 $A$ formal derived stack is a derived stack $F$ satisfying the following conditions.

1. F is nilcomplete i.e. for all $\mathbf{S p e c} A \in \mathbf{d} \mathbf{A f f}{ }_{k}$, the canonical map

$$
F(A) \longrightarrow \lim _{k} F\left(A_{\leq k}\right)
$$

(induced by the Postnikov tower of $A$ ) is an equivalence in $\mathbf{T}$.

2. $F$ is infinitesimally cohesive i.e. for all cartesian squares of almost finitely presented $k$-cdga's in non-positive degrees

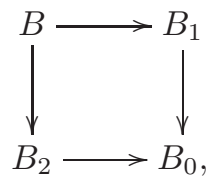

such that each $\pi_{0}\left(B_{i}\right) \longrightarrow \pi_{0}\left(B_{0}\right)$ is surjective with nilpotent kernel, then the induced square

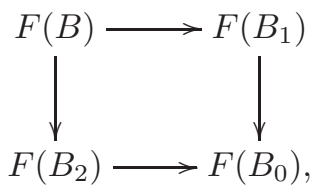

is cartesian in $\mathbf{T}$.

Remark 2.2.2 (1) The property of being infinitesimally cohesive is a derived variation of the Schlessinger condition in classical deformation theory $(\underline{\mathrm{Sch}})$. In particular, one can show that any derived Artin stack $F$ is a formal derived 
stack ([HAG-II, Appendix]), and it is actually cohesive i.e. sends any diagram as in 2.2.1 (2), with the nilpotency condition possibly omitted, to pullbacks in T ([Lu3, DAG IX, Corollary 6.5] and [Lu3, DAG XIV, Lemma 2.1.7]).

(2) A small limit of formal derived stacks is a formal derived stack.

Let the $\infty$-functor $i: \operatorname{alg}_{k}^{\text {red }} \longrightarrow$ cdga $_{k}$ be the inclusion of the full reflective sub $\infty$-category of reduced discrete objects (i.e. $R \in \mathbf{c d g a}_{k}$ such that $R$ is discrete and $R \simeq H^{0}(R)$ is a usual reduced $k$-algebra). The $\infty$-functor $i$ has a left adjoint

$$
(-)^{\text {red }}: \mathbf{c d g a}_{k} \longrightarrow \operatorname{alg}_{k}^{\text {red }}, A \longmapsto A^{\text {red }}:=H^{0}(A) / \operatorname{Nilp}\left(H^{0}(A),\right.
$$

and it is easy to verify that we get an induced $\infty$-functor $i^{*}: \mathbf{d} \mathbf{S t} t_{k} \longrightarrow \mathbf{S t}_{\mathrm{red}, k}$, where $\mathbf{S t}_{\mathrm{red}, k}$ is the $\infty$-category of stacks on $\left(\mathbf{a l g}_{k}^{\text {red }}\right)^{o p}$ for the étale topology. Now $i^{*}$ has both a right adjoint $i_{*}$, and a left adjoint $i_{!}$, both fully faithful, and $i_{!} i^{*}$ is left adjoint to $i_{*} i^{*}$.

Definition 2.2.3 1. The functor $(-)_{D R}:=i_{*} i^{*}: \mathbf{d S t}_{k} \longrightarrow \mathbf{d S t}_{k}$ is called the de Rham stack functor. By adjunction, for any $F \in \mathbf{d S t}_{k}$, we have a canonical natural map $q_{F}: F \rightarrow F_{D R}$.

2. The functor $(-)_{\text {red }}:=i_{i} i^{*}: \mathbf{d} \mathbf{S} \mathbf{t}_{k} \longrightarrow \mathbf{d S t}_{k}$ is called the reduced stack functor. By adjunction, for any $F \in \mathbf{d S t}_{k}$, we have a canonical natural map $\iota_{F}: F_{\text {red }} \rightarrow F$.

3. Let $f: F \longrightarrow G$ be a morphism in $\mathbf{d S t}_{k}$. We define the formal completion $\widehat{G}_{f}$ of $G$ along the morphism $f$ as the fibered product in $\mathbf{d S t}_{k}$ :

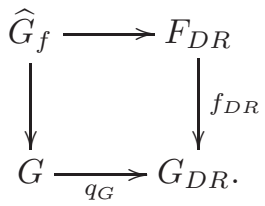

Since the left adjoint to $i$ is $(-)^{r e d}$, then it is easy to see that $F_{D R}(A) \simeq F\left(A^{\mathrm{red}}\right)$, and $(\operatorname{Spec} A)_{\mathrm{red}} \simeq \operatorname{Spec}\left(A^{\mathrm{red}}\right)$, for any $A \in \mathbf{c d g a}_{k}$. Therefore $\widehat{G}_{f}(A)=G(A) \times_{G\left(A^{\text {red }}\right)} F\left(A^{\text {red }}\right)$, for $f: F \rightarrow G$ in $\mathbf{d S t}_{k}$. We already observed that $(-)_{D R}$ is right adjoint to $(-)_{\text {red }}$, as functors $\mathbf{d} \mathbf{S} \mathbf{t}_{k} \rightarrow \mathbf{d} \mathbf{S} \mathbf{t}_{k}$. We list a few elementary properties of these constructions:

- $F_{D R}$ is a formal derived stack for any $F \in \mathbf{d S t}_{k}$.

- If $G$ is a formal derived stack, the formal completion $\widehat{G}_{f}$, along any map $f: F \rightarrow G$ in $\mathbf{d S t}_{k}$, is again a formal derived stack.

- For any $F \in \mathbf{d} \mathbf{S} \mathbf{t}_{k}$, if $j: \mathrm{t}_{0} F \rightarrow F$ denotes the canonical map in $\mathbf{d S t}_{k}$ from the truncation of $F$ to $F$, then the canonical map $\widehat{F}_{j} \rightarrow F$ is an equivalence.

Our main object of study in the next section, will be the map $q: F \rightarrow F_{D R}$. If $\mathbb{K}$ is a field, and $x: \operatorname{Spec} \mathbb{K} \rightarrow F_{D R}$ is a point (by adjunction, this is the same thing as a $\mathbb{K}$-point of $F$, since $\mathbb{K}$ is reduced), then the fiber of $q$ at $x$ is exactly the (classical) formal completion $\widehat{X_{x}}$ of $X$ at the closed point $x$. More generally, we have

Proposition 2.2.4 Let $F \in \mathbf{d S t}_{k}$, Spec $A \in \mathbf{d A f f}_{k}$, and $\bar{u}: \operatorname{Spec} A \longrightarrow F_{D R}$, corresponding to a morphism $u: \operatorname{Spec} A^{\text {red }} \longrightarrow F$. Then the base-change derived stack $F \times_{F_{D R}} \operatorname{Spec} A$ is equivalent to the formal completion $(\mathbf{S p e c} A \times F)_{(i, u)}$ of the graph morphism

$$
(i, u): \operatorname{Spec} A^{\text {red }} \longrightarrow \operatorname{Spec} A \times F,
$$

where $i: \mathbf{S p e c} A^{\text {red }} \longrightarrow \mathbf{S p e c} A$ is the natural map.

Remark 2.2.5 Suppose that $F \in \mathbf{d S t}_{k}$ has a cotangent complex (e.g. $F$ is a derived Artin stack). Then $\mathbb{L}_{F / F_{D R}}$ exists, and we have an equivalence $\mathbb{L}_{F} \simeq \mathbb{L}_{F / F_{D R}}$ in $\mathbf{Q C o h}(F)$. In fact, by the transitivity sequence for the map $q: F \rightarrow F_{D R}$. it is enough to show that $\mathbb{L}_{F_{D R}} \simeq 0$. But this follows immediately from the equivalences: $F_{D R}(A \oplus E) \simeq$ $F\left((A \oplus E)^{\text {red }}\right) \simeq F\left(A^{\text {red }}\right) \simeq F_{D R}(A)$, for any $A \in \mathbf{c d g a}_{k}^{\leq 0}$, and any $E \in \mathbf{d g}_{A}^{\leq 0}$. 


\subsubsection{Formal localization for $X \rightarrow X_{D R}$}

The general theory of formal localization is developed in detail in Section 2 of CPTVV. Instead of giving a complete account, we will content ourselves with the application of the general theory to our main case of interest, i.e. to the map $q: X \rightarrow X_{D R}$. Throughout this Section, $X$ will be derived Artin stack lfp over $k$ (hence with a perfect cotangent complex).

The map $X \rightarrow X_{D R}$ as a family of formal derived stacks. First of all, let us observe that $q: X \rightarrow X_{D R}$ is an algebraisable family of perfect formal derived stacks, i.e. for any derived point $\mathbf{S p e c} A \rightarrow X_{D R}$ the corresponding fiber $X_{A} \rightarrow \operatorname{Spec} A$ of $q$ has the following properties:

1. $X_{A}$ is a formal derived stack, and the canonical map $\left(X_{A}\right)_{\text {red }} \rightarrow \mathbf{S p e c} A_{\text {red }}$ is an equivalence in $\mathbf{d S t}_{k}$.

2. The relative cotangent complex $\mathbb{L}_{X_{A} / A}$ is perfect (by Remark 2.2.5 and base change)

3. $X_{A}$ has a cohomologically bounded above coherent cotangent complex $\mathbb{L}_{X_{A}}$ (i.e. for any $x_{B}: \operatorname{Spec} B \rightarrow X_{A}$, the fiber $x_{B}^{*} \mathbb{L}_{X_{A}}$ is a cohomologically bounded above coherent $B$-dg-module).

4. $X_{A}$ is equivalent to the formal completion of $X \times \operatorname{Spec} A$ along the map $\operatorname{Spec} A_{\text {red }} \rightarrow X \times \operatorname{Spec} A$, induced by the chosen derived point $\mathbf{S p e c} A \rightarrow X_{D R}$, and the canonical map $\operatorname{Spec} A_{\mathrm{red}} \rightarrow \operatorname{Spec} A$ (see Proposition 2.2.4).

Properties 1-3 caracterize a family of perfect formal derived stacks over $A$, while property 4 says that the family is algebraisable ( CPTVV, Section 2.1, 2.2.]).

Crystalline structure sheaf and shifted principal parts. Let us consider the $\infty$-functor

$$
\mathbb{D}: \mathbf{d A f f}_{k}^{o p} \longrightarrow \epsilon-\mathbf{c d g a}_{k}^{g r}, \quad A \longmapsto \mathbf{D R}\left(A_{\mathrm{red}} / A\right)
$$

(where $\mathbf{D R}\left(A^{\mathrm{red}} / A\right)$ is defined in Definition 1.1.1, via Remark [1.1.2). Note that $\mathbb{D}(A) \simeq \operatorname{Sym}_{A_{\mathrm{red}}}\left(\mathbb{L}_{A_{\mathrm{red}} / A}[-1]\right)$ in $\mathbf{c d g a}_{k}^{g r}$ (Section 1.1). The functor $\mathbb{D}$ satisfies étale descent, and thus we have an induced $\infty$-functor

$$
\mathbb{D}: \mathbf{d S t}_{k}^{o p} \longrightarrow \epsilon-\mathbf{c d g a}_{k}^{g r}, F \longmapsto \lim _{\mathbf{S p e c} A \rightarrow F} \mathbb{D}(A)
$$

We consider the following prestacks of mixed graded cdga's on $\mathbf{d A f f} / X_{D R}$

$$
\begin{aligned}
& \mathbb{D}_{X_{D R}}:=\mathbb{D}\left(\mathcal{O}_{X_{D R}}\right):\left(\mathbf{d A f f}_{k} / X_{D R}\right)^{o p} \longrightarrow \epsilon-\mathbf{c d g a}_{k}^{g r}, \quad\left(\operatorname{Spec} A \rightarrow X_{D R}\right) \longmapsto \mathbb{D}(A), \\
& \mathcal{P}_{X}:\left(\mathbf{d A f f}_{k} / X_{D R}\right)^{o p} \longrightarrow \epsilon-\mathbf{c d g a}_{k}^{g r}, \quad\left(\operatorname{Spec} A \rightarrow X_{D R}\right) \longmapsto \mathbb{D}\left(X_{A}\right) \text {. }
\end{aligned}
$$

Note that there is a natural equivalence $\mathcal{P}_{X}\left(\operatorname{Spec} A \rightarrow X_{D R}\right) \simeq \operatorname{Sym}_{A_{\mathrm{red}}}\left(\mathbb{L}_{\mathbf{S p e c} A_{\mathrm{red}} / X_{A}}[-1]\right)$ in $\mathbf{c d g a}_{k}^{g r}$ ([CPTVV] Proposition 2.2.6]).

Definition 2.2.6 The prestack $\mathbb{D}_{X_{D R}}$ on $X_{D R}$ is called the crystalline structure sheaf for $X$. The prestack $\mathcal{P}_{X}$ on $X_{D R}$ is called the prestack of principal parts for $X$.

Remark 2.2.7 An alternative interpretation of $\mathcal{P}_{X}$ can be given as follows. As already observed, the canonical map $X \longrightarrow X_{D R}$ realizes $X$ as a family of formal derived stacks over $X_{D R}$, namely as the family of formal completions at closed points of $X$. By Lu2 these formal completions are determined by a dg-Lie algebra $\ell_{x}$. The dg-Lie algebra $\ell_{x}$ itself does not extend globally as a sheaf of dg-Lie algebras over $X_{D R}$, simply because its underlying complex is $\mathbb{T}_{X}[-1]$, the shifted tangent complex of $X\left([\mathrm{Hen})\right.$, does not descend to $X_{D R}$. However, a remarkable consequence of derived formal localization (Theorem 2.2.12) is that the Chevalley-Eilenberg complexes of $\ell_{x}$, for $x \in X$, viewed as a graded mixed commutative dg-algebras, do glue to a global object over $X_{D R}$. This is exactly $\mathcal{P}_{X}$. Then, the Formal Localization Theorem 2.2.12 tells us exactly how to express global geometric objects on $X$ as correspondingly sheafified objects on $X_{D R}$ related to $\mathcal{P}_{X}$. 
Note that, by functoriality of $\mathbb{D}$, we have a natural morphism $\mathbb{D}_{X_{D R}} \rightarrow \mathcal{P}_{X}$ of prestacks of mixed graded cdga's on $X_{D R}$. In particular, if we consider the $\infty$-category $\mathcal{M}^{\prime}$ of prestacks on $\mathbf{d} \mathbf{A f f}{ }_{k} / X_{D R}$ with values in $\epsilon-\mathbf{d g}_{k}^{g r}$, then $\mathbb{D}_{X_{D R}} \in \mathbf{C A l g}\left(\mathcal{M}^{\prime}\right)$, and $\mathcal{P}_{X} \in \mathbb{D}_{X_{D R}} / \mathbf{C A l g}\left(\mathcal{M}^{\prime}\right)$. We let $\mathcal{M}:=\mathbb{D}_{X_{D R}}-\operatorname{Mod}_{\mathcal{M}^{\prime}}$. Then $\mathcal{P}_{X} \in \mathbf{C A l g}(\mathcal{M}) \simeq$ $\mathbb{D}_{X_{D R}} / \mathbf{C A l g}\left(\mathcal{M}^{\prime}\right)$, and, for any $n \in \mathbb{Z}$, we may consider (Section 2.1)

$$
\operatorname{Pol}^{\mathcal{M}^{\prime}}\left(\mathcal{P}_{X} / \mathbb{D}_{X_{D R}}, n\right)=\operatorname{Pol}^{\mathcal{M}}\left(\mathcal{P}_{X}, n\right) \in \mathbb{P}_{\mathcal{M}, n+1}^{g r}-\mathbf{A l g}, \quad \mathbf{D R}^{\mathcal{M}^{\prime}}\left(\mathcal{P}_{X} / \mathbb{D}_{X_{D R}}\right)=\mathbf{D R}^{\mathcal{M}}\left(\mathcal{P}_{X}\right) \in \epsilon-\mathbf{C A l g}_{\mathcal{M}}^{g r} .
$$

We will also consider the following prestacks on $\mathbf{d A f f} / X_{D R}$ obtained by Tate realizations:

$$
\begin{gathered}
\underline{\mathbf{P o l}}^{t}\left(\mathcal{P}_{X} / \mathbb{D}_{X_{D R}}, n\right):\left(\mathbf{d} \mathbf{A f f}{ }_{k} / X_{D R}\right)^{o p} \longrightarrow \mathbb{P}_{n+1}-\mathbf{c d g a}_{k}^{g r},\left(\mathbf{S p e c} A \rightarrow X_{D R}\right) \mapsto\left|\mathbf{P o l}^{\epsilon-\mathbf{d g}^{g r}}\left(\mathcal{P}_{X}(A) / \mathbb{D}_{X_{D R}}(A), n\right)\right|^{t} \\
\quad \underline{\mathbf{D R}}^{t}\left(\mathcal{P}_{X} / \mathbb{D}_{X_{D R}}\right):\left(\mathbf{d A f f}_{k} / X_{D R}\right)^{o p} \longrightarrow \epsilon-\mathbf{c d g a}_{k}^{g r},\left(\mathbf{S p e c} A \rightarrow X_{D R}\right) \mapsto\left|\mathbf{D R}^{\epsilon-\mathbf{d g}^{g r}}\left(\mathcal{P}_{X}(A) / \mathbb{D}_{X_{D R}}(A)\right)\right|^{t}
\end{gathered}
$$

Remark 2.2.8 It is worth pointing out that while $\mathcal{P}_{X}$ and $D_{X_{D R}}$ are not stacks, all $\underline{\mathbf{P o l}}^{t}\left(\mathcal{P}_{X} / \mathbb{D}_{X_{D R}}, n\right), \mathbf{D R}\left(\mathcal{P}_{X} / \mathbb{D}_{X_{D R}}\right)$, and $\underline{\mathbf{D R}}^{t}\left(\mathcal{P}_{X} / \mathbb{D}_{X_{D R}}\right)$ are stacks ([CPTVV] Corollary 2.4.9]).

Analogously (see Section 2.1), if we consider the $\infty$-category $\mathcal{M}_{\text {Ind }}^{\prime}$ of prestacks on $\mathrm{dAff}_{k} / X_{D R}$ with values in $\operatorname{Ind}\left(\epsilon-\mathbf{d g}_{k}^{g r}\right)$, then (see Section 2.1) $\mathbb{D}_{X_{D R}}(\infty) \in \mathbf{C A l g}\left(\mathcal{M}_{\text {Ind }}^{\prime}\right)$, and $\mathcal{P}_{X}(\infty) \in \mathbb{D}_{X_{D R}}(\infty) / \mathbf{C A l g}\left(\mathcal{M}_{\text {Ind }}^{\prime}\right)$. We let $\mathcal{M}_{\text {Ind }}:=\mathbb{D}_{X_{D R}}(\infty)-\operatorname{Mod}_{\mathcal{M}_{\text {Ind }}^{\prime}}$. Then $\mathcal{P}_{X}(\infty) \in \operatorname{CAlg}\left(\mathcal{M}_{\text {Ind }}\right) \simeq \mathbb{D}_{X_{D R}}(\infty) / \operatorname{CAlg}\left(\mathcal{M}_{\text {Ind }}^{\prime}\right)$, and, for any $n \in \mathbb{Z}$, we may consider

$$
\begin{gathered}
\mathbf{P o l}^{\mathcal{M}_{\text {Ind }}^{\prime}}\left(\mathcal{P}_{X}(\infty) / \mathbb{D}_{X_{D R}}(\infty), n\right)=\mathbf{P o l}^{\mathcal{M}_{\text {Ind }}}\left(\mathcal{P}_{X}(\infty), n\right) \in \mathbb{P}_{\mathcal{M}_{\text {Ind }}, n+1}^{g r}-\mathbf{A l g} \\
\mathbf{D R}^{\mathcal{M}_{\text {Ind }}^{\prime}}\left(\mathcal{P}_{X}(\infty) / \mathbb{D}_{X_{D R}}(\infty)\right)=\mathbf{D R}^{\mathcal{M}_{\text {Ind }}}\left(\mathcal{P}_{X}(\infty)\right) \in \epsilon-\mathbf{C A l g}_{\mathcal{M}_{\text {Ind }}}^{g r}
\end{gathered}
$$

And we also have the following prestacks on $\mathbf{d A f f} / X_{D R}$ obtained by standard realizations:

$$
\begin{aligned}
\underline{\mathbf{P o l}}\left(\mathcal{P}_{X}(\infty) / \mathbb{D}_{X_{D R}}(\infty), n\right):\left(\mathbf{d A f f}_{k} / X_{D R}\right)^{o p} \longrightarrow & \mathbb{P}_{n+1}-\mathbf{c d g a}_{k}^{g r}, \\
\left(\mathbf{S p e c} A \rightarrow X_{D R}\right) \longmapsto & \left|\mathbf{P o l}^{\operatorname{Ind}\left(\epsilon-\mathbf{d g}^{g r}\right)}\left(\mathcal{P}_{X}(\infty)(A) / \mathbb{D}_{X_{D R}}(\infty)(A), n\right)\right|
\end{aligned}
$$

and

$$
\begin{aligned}
\underline{\mathbf{D R}}\left(\mathcal{P}_{X}(\infty) / \mathbb{D}_{X_{D R}}(\infty)\right):\left(\mathbf{d A f f}_{k} / X_{D R}\right)^{o p} \longrightarrow & \longrightarrow-\mathbf{c d g a}_{k}^{g r} \\
\left(\mathbf{S p e c} A \rightarrow X_{D R}\right) & \longmapsto\left|\mathbf{D R}^{\operatorname{Ind}\left(\epsilon-\mathbf{d g}^{g r}\right)}\left(\mathcal{P}_{X}(\infty)(A) / \mathbb{D}_{X_{D R}}(\infty)(A)\right)\right|
\end{aligned}
$$

Remark 2.2.9 By Proposition 2.1.20 and Remark 2.1.21 we get equivalences

$$
\begin{aligned}
& \mathbf{D R}^{\mathcal{M}^{\prime}}\left(\mathcal{P}_{X} / \mathbb{D}_{X_{D R}}\right) \simeq \underline{\mathbf{D R}}{ }^{t}\left(\mathcal{P}_{X} / \mathbb{D}_{X_{D R}}\right) \simeq \underline{\mathbf{D R}}\left(\mathcal{P}_{X}(\infty) / \mathbb{D}_{X_{D R}}(\infty)\right), \\
& \underline{\mathbf{P o l}}^{t}\left(\mathcal{P}_{X} / \mathbb{D}_{X_{D R}}, n\right) \simeq \underline{\underline{\mathbf{P o l}}}\left(\mathcal{P}_{X}(\infty) / \mathbb{D}_{X_{D R}}(\infty), n\right)
\end{aligned}
$$

but notice that $\operatorname{Pol}^{\mathcal{M}^{\prime}}\left(\mathcal{P}_{X} / \mathbb{D}_{X_{D R}}, n\right)$ is not in general equivalent to the previous ones.

The Formal Localization theorem. We have already defined the mixed graded $k$-cdgas $\mathbf{D R}(X / k)$, and $\mathbf{D R}\left(X / X_{D R}\right)$ (Definition 1.1.6). It is an easy consequence of the equivalence $\mathbb{L}_{X} \simeq \mathbb{L}_{X / X_{D R}}$ (Remark 2.2.5), that $\mathbf{D R}(X / k) \simeq$ $\mathbf{D R}\left(X / X_{D R}\right)$ in $\epsilon$ - $\mathbf{c d g a}_{k}^{g r}$. We can give a similar, general definition of shifted polyvectors on $X$, at least as a graded $k$-cdga.

Definition 2.2.10 Let $F \rightarrow G$ be a map between derived stacks, both having cotangent complexes (so that $\mathbb{L}_{F / G}$ exists, too). We define the graded $k$-dg module of $n$-shifted relative polyvectors as

$$
\operatorname{Pol}(F / G, n) \simeq \bigoplus_{p \geq 0}\left(\underline{H o m}_{\mathbf{Q C o h}(F)}\left(\otimes^{p} \mathbb{L}_{F / G}, \mathcal{O}_{F}[-p n]\right)\right)^{h \Sigma_{p}} \in \mathbf{c d g a}_{k}^{g r}
$$


In the above definition, $\mathbf{Q C o h}(F)$ is regarded as a dg-category over $k$, and $\underline{H o m}_{\mathbf{Q C o h}(F)}$ denotes its $k$-dg-module of morphisms; the $\Sigma_{p}$-action on $\underline{H o m}_{\mathrm{QCoh}(F)}\left(\otimes^{p} \mathbb{L}_{F / G}, \mathcal{O}_{X}[p n]\right)$ is induced by $\Sigma_{p}$ acting in the standard way on $\otimes^{p} \mathbb{L}_{F / G}$, and via $(-1)^{n}$ times the sign representation on $\mathcal{O}_{F}[-p n]=\mathcal{O}_{F}[-n]^{\otimes^{p}}$.

Remark 2.2.11 (1) Again by Remark 2.2.5, we have an equivalence $\operatorname{Pol}\left(X / X_{D R}, n\right) \simeq \operatorname{Pol}(X / k, n)$ in $\mathbf{c d g a}_{k}^{g r}$.

(2) When $\mathbb{L}_{F / G}$ is perfect over $F$ (e.g. for $F=X$ derived Artin stack lfp over $k$, and $G=X_{D R}$ ), then we may express $\operatorname{Pol}(F / G, n)$ using the dual relative tangent complex $\mathbb{T}_{F / G}$ as (see Remark 2.1.5)

$$
\operatorname{Pol}(F / G, n) \simeq \bigoplus_{p \geq 0} \Gamma\left(F, \operatorname{Sym}^{p}\left(\mathbb{T}_{F / G}[-n]\right) \in \mathbf{c d g a}_{k}^{g r}\right.
$$

The problem with Definition 2.2.10 is that, in general, it is impossible to directly endow $\operatorname{Pol}(F(/ G, n)$, as defined, with a bracket and give it the structure of a graded $\mathbb{P}_{n+1}$-differential graded algebras over $k$. This is where the next result comes to rescue.

Theorem 2.2.12 (Formal localization for $X \rightarrow X_{D R}$ )

Let $X$ be an Artin derived stack locally of finite presentation over $k$.

1. There is a natural equivalence of $\infty$-categories

$$
\operatorname{Perf}(X) \simeq \mathcal{P}_{X}-\operatorname{Mod}_{\mathcal{M}}^{\text {perf }}
$$

where $\mathcal{M}$ was defined right after Remark 2.2.7, and $\mathcal{P}_{X}-\operatorname{Mod}_{\mathcal{M}}^{\text {perf }}$ is the full sub- $\infty$-category of $\mathcal{P}_{X}-\operatorname{Mod}_{\mathcal{M}}$, consisting of prestacks $E$ of graded mixed $\mathcal{P}_{X}$-modules on $\mathbf{d A f f} / X_{D R}$ satisfying the following two conditions:

- For all Spec $A \longrightarrow X_{D R}$, the graded mixed $\mathcal{P}_{X}(A)$-module $E(A)$ is equivalent, just as a graded $\mathcal{P}_{X}(A)$ module, to $\mathcal{P}_{X}(A) \otimes_{A_{\text {red }}} E_{0}$, for some $E_{0} \in \operatorname{Perf}\left(A_{\text {red }}\right)$.

- $E$ is quasi-coherent in the sense that: for all $\mathbf{S p e c} B \longrightarrow \mathbf{S p e c} A$ in $\mathbf{d A f f}{ }_{k} / X_{D R}$, the induced morphism $E(A) \otimes_{\mathcal{P}_{X}(A)} \mathcal{P}_{X}(B) \longrightarrow E(B)$ is an equivalence.

2. There are natural equivalences of graded mixed cdga's over $k$

$$
\mathbf{D R}\left(X / X_{D R}\right) \simeq \mathbf{D R}(X / k) \simeq \Gamma\left(X_{D R}, \underline{\mathbf{D R}}\left(\mathcal{P}_{X} / \mathbb{D}_{X_{D R}}\right)\right) \simeq \Gamma\left(X_{D R}, \underline{\mathbf{D R}}^{t}\left(\mathcal{P}_{X} / \mathbb{D}_{X_{D R}}\right)\right) .
$$

(where $\Gamma$ denotes derived global sections, i.e. $\Gamma\left(X_{D R}, \mathcal{F}\right)=\lim _{\mathbf{S p e c} A \rightarrow X_{D R}} \mathcal{F}(A)$, the limit being taken in the $\infty$-category where $\mathcal{F}$ is valued).

3. For each $n \in \mathbb{Z}$, there are natural equivalences of graded $d g$-modules over $k$

$$
\operatorname{Pol}\left(X / X_{D R}, n\right) \simeq \operatorname{Pol}(X / k, n) \simeq \Gamma\left(X_{D R}, \underline{\operatorname{Pol}}^{t}\left(\mathcal{P}_{X} / \mathbb{D}_{X_{D R}}, n\right)\right) \simeq \Gamma\left(X_{D R}, \underline{\operatorname{Pol}}\left(\mathcal{P}_{X}(\infty) / \mathbb{D}_{X_{D R}}(\infty), n\right)\right) .
$$

4. Let $\mathcal{M}_{\text {Ind }}$ be the $\infty$-category defined right after Remark 2.2.8. The natural $\infty$-functor

$$
\mathcal{P}_{X}-\operatorname{Mod}_{\mathcal{M}}^{\text {perf }} \longrightarrow \mathcal{P}_{X}(\infty)-\operatorname{Mod}_{\mathcal{M}_{\text {Ind }}}^{\text {perf }}
$$

induced by the base change $(-) \otimes k(\infty)$, is an equivalence.

5. For each $n \in \mathbb{Z}$, there are canonical equivalences in $\mathbf{T}$

$$
\operatorname{Symp}(X, n) \simeq \operatorname{Symp}\left(\mathcal{P}_{X} / \mathbb{D}_{X_{D R}}, n\right) \simeq \operatorname{Symp}\left(\mathcal{P}_{X}(\infty) / \mathbb{D}_{X_{D R}}(\infty), n\right)
$$

where $\operatorname{Symp}\left(\mathcal{P}_{X} / \mathbb{D}_{X_{D R}}, n\right)$ and $\operatorname{Symp}\left(\mathcal{P}_{X}(\infty) / \mathbb{D}_{X_{D R}}(\infty), n\right)$ are defined as in Definition 2.1.2 (with $\mathcal{M}$ and $\mathcal{M}_{\text {Ind }}$, respectively, as defined in the previous paragraph).

The proof of Theorem 2.2.12 can be found in CPTVV, Corollary 2.4.12, Proposition 2.4.15]. 
Remark 2.2.13 Let us explain why the formal localization Theorem 2.2.12 is important and useful.

(1) Points 1 and 4 in Theorem 2.2.12 are absolutely crucial since they allow us to completely recover perfect complexes on $X$ as certain, explicitly identified, mixed graded modules over $\mathcal{P}_{X}$ or $\mathcal{P}_{X}(\infty)$. This makes manipulations on perfect complexes much easier, and will allow us to go from a quantization of $\mathcal{P}_{X}(\infty)$ to a quantization of $\operatorname{Perf}(X)$ (see Section 3.1).

(2) Point 2 in the above Theorem lets us completely recover (with its full structure of mixed graded algebra) the descent-theoretic definition 1.1.6 of the de Rham algebra on $X$ in terms of $\mathcal{P}_{X} / \mathbb{D}_{X_{D R}}$.

(3) Point 3 is important because it allows us not only to recover the 'geometrical" polyvectors of Definition 2.2.10 but also to establish a full graded $\mathbb{P}_{n+1}$-algebra structure on them. This is essential in order to be able to define shifted Poisson structures on $X$ (Definition 2.3.1).

(4) The last point of Theorem 2.2.12 lets us completely recover shifted symplectic forms on $X$ in terms of shifted symplectic form on $\mathcal{P}_{X} / \mathbb{D}_{X_{D R}}\left(\right.$ or $\left.\mathcal{P}_{X}(\infty) / \mathbb{D}_{X_{D R}}(\infty)\right)$. This have the effect to enable a definition of shifted Poisson and shifted symplectic structures in terms of the very same object $\mathcal{P}_{X} / \mathbb{D}_{X_{D R}}\left(\right.$ or $\left.\mathcal{P}_{X}(\infty) / \mathbb{D}_{X_{D R}}(\infty)\right)$, thus opening the way for a comparison between them (see Theorem 2.4.2).

\subsection{Shifted Poisson structures}

We are finally ready to define shifted Poisson structures on a derived Artin stack $X$ lfp over $k$. In the previous Section (Definition 2.2.6), we constructed two prestacks $\mathbb{D}_{X_{D R}}$, and $\mathcal{P}_{X}:=\mathbb{D}_{X / X_{D R}}$ of graded mixed cdga's on $X_{D R}$, together with a map of prestacks $\mathbb{D}_{X_{D R}} \rightarrow \mathcal{P}_{X}$, exhibiting $\mathcal{P}_{X}$ as a prestack of $\mathbb{D}_{X_{D R}}$-linear graded mixed cdga's on $X_{D R}$. By passing to Tate realization, we obtain the prestack $\underline{\text { Poll }}^{t}\left(\mathcal{P}_{X} / \mathbb{D}_{X_{D R}}, n\right)$ of graded $\mathbb{P}_{n+1^{-}}$-cdga' on $\mathbf{d A f f} / X_{D R}$.

Definition 2.3.1 If $X$ is a derived Artin stack lfp over $k$, and $n \in \mathbb{Z}$, we define

- the graded $\mathbb{P}_{n+1}$-cdga over $k, \operatorname{Pol}(X, n):=\Gamma\left(X_{D R}, \underline{\text { Poll }}^{t}\left(\mathcal{P}_{X} / \mathbb{D}_{X_{D R}}, n\right)\right)$ of $n$-shifted polyvectors on $X$;

- the space $\operatorname{Poiss}(X, n):=\operatorname{Map}_{\mathbf{d g L i e}_{k}^{g r}}(k(2)[-1], \operatorname{Pol}(X, n+1)[n+1])$ of $n$-shifted Poisson structures on $X$. An $n$-shifted Poisson structure on $X$ is an element $\pi \in \pi_{0}(\operatorname{Poiss}(X, n))$.

In the second item of the previous definition, $\mathbf{d g L i e}_{k}^{g r}$ is the $\infty$-category of graded $k$-linear dg-Lie algebras, and $k(2)[-1]$ denotes $k$ sitting in cohomological degree 1 , in weight degree 2 , endowed with trivial bracket and trivial differential.

Since $\mathbb{L}_{X}$ is perfect, by Theorem 2.2.12 (3) and Remark 2.2.11, there is an equivalence

$$
\operatorname{Pol}(X, n) \simeq \operatorname{Pol}(X / k, n) \simeq \bigoplus_{p \geq 0} \Gamma\left(X, \operatorname{Sym}^{p}\left(\mathbb{T}_{X}[-n]\right)\right.
$$

of graded mixed dg-modules over $k$. This justifies the use of the word polyvectors for $\operatorname{Pol}(X, n)$.

The intuition behind our definition of Poiss $(X, n)$ is that if $X$ is a smooth scheme, $n=0$, and we replace $\operatorname{Map}_{\mathbf{d g L i e}_{k}^{g r}}$ with the usual, strict $\operatorname{Hom}_{\mathrm{dgli}_{k}^{g r}}$, one gets that an element in $\operatorname{Hom}_{\mathrm{dgli}_{k}^{g r}}(k(2)[-1], \operatorname{Pol}(X, n+1)[n+1])$ is exactly a bivector field $\pi$, such that $[\pi, \pi]=0,[-,-]$ being the usual Schouten-Nijenhuis bracket on algebraic polyvector fields on $X$; in other words, such a $\pi$ is a usual algebraic Poisson bivector on $X$. See also Example 2.3.3 (1) below.

We can give an alternative description of the space $\operatorname{Poiss}(X, n)$. Recall from the previous section that the stabilized versions $\mathbb{D}_{X_{D R}}(\infty)$ and $\mathcal{P}_{X}(\infty)$ of $\mathbb{D}_{X_{D R}}$ and $\mathcal{P}_{X}$ are both prestacks of commutative monoid objects in $\operatorname{lnd}\left(\epsilon-\mathbf{d g}^{g r}\right)$ on $X_{D R}$, and that there is an analogous canonical map $\mathbb{D}_{X_{D R}}(\infty) \rightarrow \mathcal{P}_{X}(\infty)$. We let $\mathbb{P}_{n+1}\left(\mathcal{P}_{X}(\infty) / \mathbb{D}_{X_{D R}}(\infty)\right)$ be the space of those $\mathbb{P}_{n+1}$-algebras structures on $\mathcal{P}_{X}(\infty)$, in the $\infty$-category $\mathcal{M}$ of prestacks, on $X_{D R}$, of $\mathbb{D}_{X_{D R}}(\infty)$-modules inside $\operatorname{lnd}\left(\epsilon-\mathbf{d g}^{g r}\right)$, which are compatible the given commutative algebra structure on $\mathcal{P}_{X}(\infty)$ in $\mathcal{M}$. An elaboration of Corollary 2.1.11yields the following comparison

Theorem 2.3.2 ([CPTVV, Theorem 3.1.2]) For any derived Artin stack $X$ lfp over $k$, and any $n \in \mathbb{Z}$, we have a canonical equivalence $\operatorname{Poiss}(X, n) \simeq \mathbb{P}_{\mathcal{M}, n+1}\left(\mathcal{P}_{X}(\infty) / \mathbb{D}_{X_{D R}}(\infty)\right)$ in $\mathbf{T}$. 
This theorem should be viewed as a vast generalization to derived Artin stacks of the equivalence between the notion of Poisson bivectors on $X$ and Poisson brackets on $\mathcal{O}_{X}$, well-known for smooth schemes $X$.

Examples 2.3.3 (1) If $X$ is a smooth scheme, then the space $\operatorname{Poiss}(X, 0)$ is discrete and equivalent to the vector space of usual algebraic Poisson brackets on $\mathcal{O}_{X}$.

(2) If $G$ is a reductive group scheme over $k$, and $\mathfrak{g}$ its Lie algebra, then one has ([CPTVV, 3.1])

$$
\pi_{0}(\operatorname{Poiss}(\mathrm{B} G, n)) \simeq \begin{cases}\wedge_{k}^{3}(\mathfrak{g})^{G}, & n=1 \\ \operatorname{Sym}_{k}^{2}(\mathfrak{g})^{G}, & n=2 \\ 0, & n \neq 1,2\end{cases}
$$

The comparison theorem in the next section, together with the coisotropic (combined with the results of Section 1.2), and the intersection theorem in Section 2.5, will provide more examples of shifted Poisson structures.

\subsection{Comparison between non degenerate shifted Poisson structures and shifted sym- plectic structures}

In this Section, we establish a derived analog of the usual equivalence between classical non-degenerate Poisson structures and symplectic structures.

Let $X$ be a derived Artin stack lfp over $k, n \in \mathbb{Z}$, and $\pi \in \pi_{0}(\operatorname{Poiss}(X, n))$ be an $n$-shifted Poisson structure on $X$ (Definition 2.3.1). By considering the "forgetful" map

$$
\operatorname{Map}_{\mathbf{d g L i e}_{k}^{g^{r}}}(k(2)[-1], \operatorname{Pol}(X, n+1)[n+1]) \longrightarrow \operatorname{Map}_{\mathbf{d g}_{k}^{g r}}(k(2)[-1], \operatorname{Pol}(X, n+1)[n+1]),
$$

together with the equivalence in $\mathbf{d g}_{k}^{g r}$

$$
\operatorname{Pol}(X, n+1)[n+1] \simeq \bigoplus_{p} \Gamma\left(X, \operatorname{Sym}_{\mathcal{O}_{X}}^{p}\left(\mathbb{T}_{X}[-n-1]\right)[n+1]\right.
$$

(see Remark 2.2.11),$\pi$ induces a morphism $k(2) \rightarrow \oplus_{p} \Gamma\left(X, \operatorname{Sym}_{\mathcal{O}_{X}}^{p}\left(\mathbb{T}_{X}[-n-1]\right)[n+2]\right.$ in $\mathbf{d g}_{k}^{g r}$, and thus defines an element $\alpha_{\pi} \in H^{-n}\left(X, \Phi_{n}^{(2)}\left(\mathbb{T}_{X}\right)\right)$, where

$$
\Phi_{n}^{(2)}\left(\mathbb{T}_{X}\right):=\left\{\begin{array}{l}
S y m_{\mathcal{O}_{X}}^{2} \mathbb{T}_{X}, \text { if } \mathrm{n} \text { is odd } \\
\wedge_{\mathcal{O}_{X}}^{2} \mathbb{T}_{X}, \text { if } \mathrm{n} \text { is even }
\end{array}\right.
$$

We denote by $\pi^{\sharp}$ the map $\mathbb{L}_{X} \rightarrow \mathbb{T}_{X}[-n]$ induced, via adjunction, by $\alpha_{\pi}$.

Definition 2.4.1 Let $X$ be a derived Artin stack lfp over $k$, and $n \in \mathbb{Z}$. An n-shifted Poisson structure $\pi \in$ $\pi_{0}(\operatorname{Poiss}(X, n))$ is non-degenerate if the induced map $\pi^{\sharp}: \mathbb{L}_{X} \rightarrow \mathbb{T}_{X}[-n]$ is an equivalence. We denote by Poiss ${ }^{n d}(X, n)$ the subspace of Poiss $(X, n)$ whose connected components are non-degenerate $n$-shifted Poisson structures on $X$.

We are now ready to construct the comparison map between the space of shifted Poisson structures and the space of shifted symplectic structures on derived Artin stacks.

Recalling from the previous Section, let $\mathcal{M}_{\text {Ind }}^{\prime}$ is the category of prestacks on $\mathbf{d A f f} / X_{D R}$ with values in $\operatorname{lnd}\left(\epsilon-\mathbf{d g}_{k}^{g r}\right)$, and $\mathcal{M}_{\text {Ind }}$ the category of $D_{X_{D R}}(\infty)$-modules in $\mathcal{M}^{\prime}$. Then $A:=\mathcal{P}_{X}(\infty)$ belongs to $\mathbf{C A l g} \lg _{\mathcal{M}}$. By Proposition 2.1.18 and Th. 2.3.2 we have an equivalence $\mathbb{P}_{\mathcal{M}, n+1}^{n d}\left(\mathcal{P}_{X}(\infty) / \mathbb{D}_{X_{D R}}(\infty)\right) \simeq \operatorname{Poiss}^{n d}(X, n)$, where $\mathbb{P}_{n+1}^{n d}\left(\mathcal{P}_{X}(\infty) / \mathbb{D}_{X_{D R}}(\infty)\right)$ is the subspace of non degenerate $\mathbb{P}_{n+1}$-algebra structures on $\mathcal{P}_{X}(\infty) / \mathbb{D}_{X_{D R}}(\infty)$, compatible with the underlying commutative $\mathbb{D}_{X_{D R}}(\infty)$-algebra structure on $\mathcal{P}_{X}(\infty)$.

Recall from Proposition 2.1.17, that for any $\mathcal{M}$, and any $A \in \mathbf{C A l g}_{\mathcal{M}}$ with a dualizable cotangent complex, we have a map $\mathbb{P}_{\mathcal{M}, n+1}^{n d}(A) \rightarrow \operatorname{Sympl}(A, n)$, from the moduli space of those non-degenerate $\mathbb{P}_{n+1}$-algebra structures in $\mathcal{M}$ on $A$ which are compatible with the given commutative algebra structure on $A$, to the moduli space of $n$-shifted symplectic structures on $A$. With our current choice of $\mathcal{M}$, we thus get a map

$$
\operatorname{Poiss}^{n d}(X, n) \simeq \mathbb{P}_{\mathcal{M}, n+1}^{n d}\left(\mathcal{P}_{X}(\infty) / \mathbb{D}_{X_{D R}}(\infty)\right) \longrightarrow \operatorname{Sympl}\left(\mathcal{P}_{X}(\infty) / \mathbb{D}_{X_{D R}}(\infty), n\right)
$$


But by Theorem 2.2.12, we have $\operatorname{Sympl}\left(\mathcal{P}_{X}(\infty) / \mathbb{D}_{X_{D R}}(\infty), n\right) \simeq \operatorname{Sympl}(X, n)$, so we obtain a comparison map

$$
\psi: \operatorname{Poiss}^{n d}(X, n) \longrightarrow \operatorname{Sympl}(X, n)
$$

from the space of non-degenerate $n$-shifted Poisson structures on $X$, to the space of $n$-shifted symplectic structures on $X$. One of the main result of [CPTVV], and the key to quantize all the shifted symplectic moduli spaces constructed in Section 1.2, is the following

Theorem 2.4.2 [CPTVV] Theorem 3.2.4] Let $X$ be a derived Artin stack lfp over $k$, and $n \in \mathbb{Z}$. The canonical map $\psi: \operatorname{Poiss}^{n d}(X, n) \rightarrow \operatorname{Sympl}(X, n)$ is an equivalence in $\mathbf{T}$.

In spite of being expected, and somehow very natural, Theorem 2.4.2 has a rather technical and non-trivial proof. It is not too difficult showing that $\psi$ induces isomorphisms on all the homotopy groups $\pi_{i}$ 's for $i \geq 1$. More difficult is proving that $\psi$ is an isomorphism on $\pi_{0}$ : this is achieved in CPTVV by first showing that the functors $A \mapsto \mathbb{P}_{\mathcal{M}, n+1}^{n d}\left(\mathcal{P}_{X}(\infty)(A) / \mathbb{D}_{X_{D R}}(\infty)(A)\right)$, and $A \mapsto \operatorname{Sympl}\left(\mathcal{P}_{X}(\infty)(A) / \mathbb{D}_{X_{D R}}(\infty)(A), n\right)$ are both formal derived stacks (Definition 2.2.1), and then showing that it is enough to prove the isomorphism on $\mathbf{d A f f}{ }^{\text {red }} / X_{\text {red }}$. This reduced case is then specifically handled by using pairings and copairings on $L_{\infty}$-algebras, the $L_{\infty}$-algebra being given, for any $u$ : Spec $A \rightarrow X$, with $A$ reduced, by an $L_{\infty}$-model for $\left(u^{*} \mathbb{L}_{X}\right)^{\vee}[-1]$. Similar techniques have been used in Co-Gwi].

\subsection{Coisotropic structures}

In this section we discuss briefly the notion of a coisotrpic structure on a map to a general $n$-shifted Poisson target. This is analogous to the notion of a lagrangian structure from Definition 1.2.3 A new feature of the Poisson context is that the definition of coisotropic structure itself requires a non-trivial statement - Rozenblyum's additivity theorem. This additivity theorem asserts that for every $n \geq 1$ and every symmetric monoidal $\infty$-category $\mathcal{M}$, satisfying our standard hypotheses, there is a natural equivalence between the $\infty$-category $\mathbb{P}_{\mathcal{M}, n+1}-\mathbf{A l g}$ and the $\infty$-category $\operatorname{Alg}\left(\mathbb{P}_{\mathcal{M}, n}-\mathbf{C A l g}\right)$ of unital and associative algebras in the category $\mathbb{P}_{\mathcal{M}, n}-\mathbf{A l g} 6$ (note that $\mathbb{P}_{\mathcal{M}, n}$ is a Hopf operad, hence $\mathbb{P}_{\mathcal{M}, n}$ - Alg inherits a natural symmetric monoidal structure).

The additivity equivalence is functorial in $\mathcal{M}$, with respect to symmetric monoidal $\infty$-functors, and commutes with the forgetful functors to $\mathcal{M}$ (see CPTVV, Remark 3.4.2] for more details). The main utility of the additivity theorem is that it allows us to make sense of $\mathbb{P}_{n+1}$-structures on morphisms between commutative algebras in $\mathcal{M}$. More precisely, if we write $\mathbb{P}_{\mathcal{M},(n+1, n)}-\mathbf{A l g}$ for the $\infty$-category of pairs $(A, B)$ consisting of an object $A \in \mathbf{A} \lg \left(\mathbb{P}_{\mathcal{M}, n}-\mathbf{A l g}\right)$ and an $A$-module $B$ in $\mathbb{P}_{\mathcal{M}, n}-\mathbf{A l g}_{\mathcal{M}}$, then by the additivity theorem $\mathbb{P}_{\mathcal{M},(n+1, n)}-\mathbf{A l g}$ comes equipped with two forgetful $\infty$-functors $(A, B) \mapsto A$, and $(A, B) \mapsto B$ to $\mathbb{P}_{\mathcal{M}, n+1}-\mathbf{A l g}$, and $\mathbb{P}_{\mathcal{M}, n}-\mathbf{A l g}$, respectively. Furthermore, the forgetful functor $\mathbb{P}_{\mathcal{M}, n}-\mathbf{A l g} \rightarrow \mathbf{A l g}_{\mathcal{M}}$ induces a natural forgetful functor from $\mathbb{P}_{\mathcal{M},(n+1, n)}-\mathbf{A l g}_{\mathcal{M}}$ to the $\infty$ category of pairs $(A, B)$ where $A \in \mathbf{A} \lg \left(\mathbf{C A l g} \lg _{\mathcal{M}}\right)$ and $B$ is an $A$-module in $\mathbf{C A} \mathbf{l g}_{\mathcal{M}}$. The $\infty$-category of such pairs is naturally equivalent to the $\infty$-category $\left.\mathbf{M o r}(\mathbf{C A l g})_{\mathcal{M}}\right)$ of morphisms in $\mathbf{C A l g} \mathbf{I g}_{\mathcal{M}}$. In particular, given a morphism

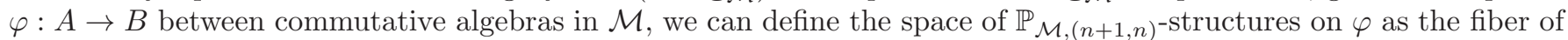
the $\infty$-functor $\mathbb{P}_{\mathcal{M},(n+1, n)}-\mathbf{A l g} \rightarrow \operatorname{Mor}\left(\mathbf{A l g}_{\mathcal{M}}\right)$ over $\varphi$. We will write

$$
\mathbb{P}_{\mathcal{M},(n+1, n)}(\varphi):=\mathbb{P}_{\mathcal{M},(n+1, n)}-\mathbf{A l g} \times_{\operatorname{Mor}\left(\mathbf{C A l g}_{\mathcal{M}}\right)}\{\varphi\}
$$

for this space. Note that by construction the space $\mathbb{P}_{\mathcal{M},(n+1, n)}(\varphi)$ projects naturally both to the space $\mathbb{P}_{\mathcal{M}, n+1}(A)$ of $\mathbb{P}_{n+1}$-structures on the source $A$, and to the space $\mathbb{P}_{\mathcal{M}, n}(B)$ of $\mathbb{P}_{n}$-structures on the target $B$.

Let $f: X \rightarrow Y$ is a morphism of derived stacks locally of finite presentation over $k$. We specialize the construction above to the following case:

- $\mathcal{M}:=\mathcal{M}_{X, \text { Ind }}$ is the $\infty$-category defined in Section 2.4 (i.e. if $\mathcal{M}_{X, \text { Ind }}^{\prime}$ is the $\infty$-category of prestacks on $\mathrm{dAff} / X_{D R}$ with values in $\operatorname{Ind}\left(\epsilon-\mathbf{d g}_{k}^{g r}\right)$, then $\mathcal{M}_{X \text {,Ind }}$ is the $\infty$-category of $D_{X_{D R}}(\infty)$-modules in $\left.\mathcal{M}_{X, \text { Ind }}^{\prime}\right)$.

- $\varphi$ is the induced map $f_{\mathcal{P}}^{*}: f_{D R}^{*}\left(\mathcal{P}_{Y}(\infty)\right) \rightarrow \mathcal{P}_{X}(\infty)$.

Note that the map $f_{D R}^{*}\left(\mathbb{D}_{Y_{D R}}(\infty)\right) \rightarrow \mathbb{D}_{X_{D R}}(\infty)$ is an equivalence and so $f_{\mathcal{P}}^{*}$ may indeed be considered as a morphism in $\mathrm{CA} \lg _{\mathcal{M}}$.

Now, if $Y$ is endowed with an $n$-shifted Poisson structure $\pi$, then $\mathcal{P}_{Y}(\infty)$ is canonically an object in $\mathbb{P}_{\mathcal{M}_{Y, \text { Ind }}, n+1}-$ Alg (Theorem 2.3.2), and thus its pull-back $f_{D R}^{*}\left(\mathcal{P}_{Y}(\infty)\right)$ is canonically an object in $\mathbb{P}_{\mathcal{M}, n+1}-\mathbf{A l g}$. We denote this

${ }^{6}$ The same result holds for the operads $\mathbb{E}_{n}$ of little $n$-disks, and it is known as Dunn-Lurie additivity [Lu4 5.1.2.2]. 
object by $\mathcal{P}_{Y, f}^{\pi}$ (in order to distinguish it from $f_{D R}^{*}\left(\mathcal{P}_{Y}(\infty)\right)$ as an object in $\mathbf{C A l g} \lg _{\mathcal{M}}$ ). To ease notation, we will write $\mathbb{P}_{(n+1, n)}\left(f_{\mathcal{P}}^{*}\right)$ for $\mathbb{P}_{\mathcal{M},(n+1, n)}\left(f_{\mathcal{P}}^{*}\right)$, and $\mathbb{P}_{n+1}\left(f_{D R}^{*}\left(\mathcal{P}_{Y}(\infty)\right)\right)$ for $\mathbb{P}_{\mathcal{M}, n+1}\left(f_{D R}^{*}\left(\mathcal{P}_{Y}(\infty)\right)\right)$. So it makes sense to consider the fiber of the projection map $\mathbb{P}_{(n+1, n)}\left(f_{\mathcal{P}}^{*}\right) \rightarrow \mathbb{P}_{n+1}\left(f_{D R}^{*}\left(\mathcal{P}_{Y}(\infty)\right)\right)$ over $\mathcal{P}_{Y, f}^{\pi}$.

Definition 2.5.1 Let $f: X \rightarrow Y$ be a morphism of derived stacks locally of finite presentation over $k$ and assume that $Y$ is equipped with an n-shifted Poisson structure $\pi$. The space of coisotropic structures on $f$ relative to $\pi$ is the fiber

$$
\operatorname{Cois}(f, \pi):=\mathbb{P}_{(n+1, n)}\left(f_{\mathcal{P}}^{*}\right) \times_{\mathbb{P}_{n+1}\left(f_{D R}^{*}\left(\mathcal{P}_{Y}(\infty)\right)\right)}\left\{\mathcal{P}_{Y, f}^{\pi}\right\}
$$

$A$ coisotropic structure on $f$ relative to $\pi$ is an element in $\pi_{0} \operatorname{Cois}(f, \pi)$.

In other words, a coisotropic structure on $f: X \rightarrow Y$ consists of the datum of a $\mathbb{D}_{X_{D R}}(\infty)$-linear $\mathbb{P}_{n}$-algebra structure on $\mathcal{P}_{X}(\infty)$ (the target of $f_{\mathcal{P}}^{*}$ ), together with a suitably compatible structure of module over $\mathcal{P}_{Y, f}^{\pi}$ (the source of $f_{\mathcal{P}}^{*}$ ), inside the $\infty$-category of $\mathbb{D}_{X_{D R}}(\infty)$-linear graded mixed $\mathbb{P}_{n}$-algebras on $X_{D R}$.

Remark 2.5.2 This notion of coisotropic structure has the expected geometric behavior:

(i) Using the other projection map $\mathbb{P}_{(n+1, n)}\left(f_{\mathcal{P}}^{*}\right) \rightarrow \mathbb{P}_{n}\left(\mathcal{P}_{X}(\infty)\right.$ ) (i.e. the map keeping only the target of $f_{\mathcal{P}}^{*}$ ), and Theorem 2.3.2. we get that a choice of a coisotropic structure on $f: X \rightarrow Y$ relative to an $n$-shifted Poisson structure $\pi$ on $Y$, tautologically induces an $(n-1)$-shifted Poisson structure on the source $X$.

(ii) If $\pi_{\omega}$ is a non-degenerate Poisson structure corresponding to an $n$-shifted symplectic structure $\omega$ on $Y$, then, keeping the notations of Definition 2.5.1] one expects a natural equivalence of spaces $\operatorname{Lag}(f, \omega) \cong \operatorname{Cois}(f, \pi)^{\text {nd }}$ between the space of lagrangian structures on $f$ (see Definition 1.2.3) and the space of suitably non-degenerate coisotropic structures on $f$. This is being investigated by Melani and Safronov (see [Me-Sa]).

(iii) The Lagrangian intersection theorem, Theorem 1.2.5 was recently extended to the Poisson context in Me-Sa. Suppose $(Y, \pi)$ be an $n$-shifted Poisson Artin stack locally of finite presentation over $k$. Let $f_{i}: X_{i} \rightarrow Y$, $i=1,2$ be maps of derived Artin stacks each endowed with coisotropic structures relative to $\pi$. Then, Melani and Safronov prove that the derived fiber product $X_{1} \times_{Y} X_{2}$ has a natural, induced $(n-1)$-shifted Poisson structure such that the natural map $X_{1} \times_{Y} X_{2} \rightarrow X_{1} \times X_{2}$ is a morphism of $(n-1)$-shifted Poisson stacks, where in the target $X_{2}$ is endowed with the $(n-1)$-shifted Poisson structure from point (i) above, and $X_{1}$ with the corresponding opposite $(n-1)$-shifted Poisson structure (see [Me-Sa for details). A classical, i.e. 0-shifted, and purely cohomological precursor of this result was proved in Gi-Ba. Aside from its conceptual significance, the coisotropic intersection theorem of $\mathrm{Me}-\mathrm{Sa}$ has many purely utilitarian corollaries. It allows us to extend the list of examples at the end of Section 2.3. by providing many more examples of shifted Poisson structures on moduli stacks, hence of moduli stacks admitting natural deformation quantizations (see Section 3). For instance, recently Spaide $\mathrm{Spa}$ applied coisotropic inersections to construct and characterize shifted Poisson structures on moduli spaces of framed sheaves in arbitrary dimension as well as on the moduli of monopoles.

\section{Deformation quantization}

Recall that for an ordinary smooth scheme $X$ over $k$, a classical (unshifted) Poisson structure $\pi$ can be viewed as the infinitesimal to the deformation of $\mathcal{O}_{X}$ as a sheaf of associative algebras on $X$. According to the algebraic deformation quantization results of Kontsevich [Ko1 and Yekutieli Ye every ordinary Poisson scheme $(X, \pi)$ admits a quantization as a stack of algebroids. That is we can always find a stack of algebroids $\mathcal{X}$ defined over $k[[\hbar]]$ with $(\mathcal{X} \bmod \hbar)=X$ and with infinitesimal $\pi$. Moreover [Ko1, Ye all possible quantizations with a given infinitesimal depend on a choice of a formality quasi-isomorphism (Drinfeld associator) and are classified by deformation of $(X, \pi)$ as a Poisson scheme over $k[[\hbar]]$. In particular the trivial Poisson deformation corresponding to the $k[[\hbar]]$-linear Poisson bivector $\hbar \cdot \pi$ gives rise to a preferred quantization of $(X, \pi)$. This preferred quantization is Kontsevich's canonical quantization, or in the case of a non-degenerate $\pi$ is the algebraic Fedosov canonical quantization of Bezrukavnikov-Kaledin [Bez-Ka].

In this section we discuss the extension of the deformation quantization problem to shifted Poisson structures on derived Artin stacks. We argue that the canonical $n$-shifted quantization always exists as long as $n \neq 0$ and again depends on the choice of a Drinfeld associator. Ineterestingly enough the special case when $n=0$ remains the hardest case and the best existing quantization results are still those of [Ko1, Ye]. The natural question of extending the Ko1, Ye quantization of smooth Poisson schemes to 0-shifted Poisson derived Artin stacks requires new ideas and will not be treated here. 


\subsection{Weak and strong quantization}

Informally, shifted Poisson structures arise when we study deformations of $X$ in which we allow only partial noncommutativity in the deformed product structure. More precisely, an $n$-shifted Poisson structure can be viewed as the infinitesimal for deforming the commutative $\left(=\mathbb{E}_{\infty}\right)$ algebra structure on $\mathcal{P}_{X}(\infty)$ to an $\mathbb{E}_{n+1}$-algebra structure.

To spell this out, recall that for $n \geq 1$ the operad $\mathbb{E}_{n}$ of chains little $n$-dimensional disks is a $k$-linear dg-operad which is given by the chain complexes $C \bullet\left(F M_{n}, k\right)$ of the Fulton-MacPherson's topological operad $F M_{n}$. By definition, the space of operations of $F M_{n}$ labeled by a finite set $I$ is the Fulton-MacPherson compactified configuration space $F M_{n}(I)$ of $I$-labeled configurations of points in $\mathbb{R}^{n}$. For $n \geq 2$ the Postnikov tower of the spaces $F M_{n}(I)$ induces a filtration of $\mathbb{E}_{n}$ whose associated graded is the graded $k$-linear homology operad $H_{\bullet}\left(F M_{n}, k\right)$ of $F M_{n}$ which is known to be the operad $\mathbb{P}_{n}$ controlling $(n-1)$-shifted Poisson algebras. We can now apply the Rees construction to the filtration on $\mathbb{E}_{n}$ to obtain a dg-operad $\mathbb{B D}_{n}$ (for Beilinson-Drinfeld) which is linear over $k[h]$ and deforms the filtered operad $\mathbb{E}_{n}$ to its associated graded $\mathbb{P}_{n}$. This deformation makes sense for $n=1$ as well. In this case, $\mathbb{E}_{1}$ is the operad controlling associative algebra structures. For every finite $I$, the $k$-module of operations $\mathbb{E}_{1}(I)$ labeled by $I$ is the non-commutative polynomial algebra $k\left\langle x_{i} \mid i \in I\right\rangle$ which is naturally filtered by monomial degree. The associated graded to this filtration is the commutative polynomial algebra $k\left[x_{i} \mid i \in I\right]$ equipped with the induced Lie bracket, i.e. we have gr $\mathbb{E}_{1}(I)=P_{1}(I)$. Again applying the Rees construction to the filtration gives a $k[h]$-linear operad $\mathbb{B D}_{1}$ which interpolates between $\mathbb{E}_{1}$ and $\mathbb{P}_{1}$. The difference between this case and the case $n \geq 2$ is that $\mathbb{P}_{1}$ is not the homology of $\mathbb{E}_{1}$. In fact $\mathbb{E}_{1}$ is already formal and isomorphic to $H_{\bullet}\left(F M_{1}, k\right)$.

Nevertheless, for any $n \geq 1$ we constructed a $k[\hbar]$-linear dg-operad operad $\mathbb{B D}_{n}$ such that $\mathbb{B D}_{n} \otimes_{k[\hbar]} k \cong \mathbb{P}_{n}$ and $\mathbb{B D}_{n} \otimes_{k[\hbar]} k\left[\hbar, \hbar^{-1}\right] \cong \mathbb{E}_{n}\left[\hbar, \hbar^{-1}\right]$. With this in place, we are now ready to formulate the quantization problem in the shifted setting. Suppose $X$ is a derived Artin stack, locally of finite presentation over $k$. We use again the notations from Section 2.2.2: $\mathcal{M}_{\text {Ind }}^{\prime}$ is the $\infty$-category of prestacks on $\mathbf{d} \mathbf{A f f}{ }_{k} / X_{D R}$ with values in $\operatorname{Ind}\left(\epsilon-\mathbf{d g}_{k}^{g r}\right)$, so that $\mathbb{D}_{X_{D R}}(\infty) \in \mathbf{C A} \lg \left(\mathcal{M}_{\text {Ind }}^{\prime}\right), \mathcal{P}_{X}(\infty) \in \mathbb{D}_{X_{D R}}(\infty) / \mathbf{C A l g}\left(\mathcal{M}_{\text {Ind }}^{\prime}\right)$, and we define $\mathcal{M}_{\text {Ind }}:=\mathbb{D}_{X_{D R}}(\infty)-\operatorname{Mod}_{\mathcal{M}_{\text {Ind }}^{\prime}}$.

By Theorem 2.3.2 specifying an $n$-shifted Poisson structure $\pi$ on $X$ is equivalent to specifying a $\mathbb{P}_{\mathcal{M}_{\text {Ind }}, n+1 \text {-algebra }}$ structure on the Tate stack of principal parts $\mathcal{P}_{X}(\infty)$, compatible with its given commutative $\mathbb{D}_{X_{D R}}(\infty)$-algebra structure. Suppose $n \geq 0$, then we have two flavors of the quantization problem:

(strong quantization) Construct a $\mathbb{D}_{X_{D R}}(\infty)$-linear $\mathbb{B D}_{n+1}$-algebra structure on $\mathcal{P}_{X}(\infty) \otimes k[\hbar]$, such that after tensoring with $\otimes_{k[\hbar]} k$ we get the $\mathbb{P}_{n+1}$-structure given by $\pi$.

(weak quantization) Construct a $\mathbb{B D}_{n}$-monoidal structure on the $\infty$-category (Theorem 2.2.12 (1) and (4))

$$
\operatorname{Perf}(X) \otimes_{k} k[\hbar] \cong \mathcal{P}_{X}(\infty)-\operatorname{Mod}_{\mathcal{M}_{\text {Ind }}}^{\text {perf }} \otimes_{k} k[\hbar]
$$

which after $\otimes_{k[\hbar]} k$ recovers the $\mathbb{P}_{n}$-monoidal structure on $\mathcal{P}_{X}(\infty)-\operatorname{Mod}_{\mathcal{M}_{\text {Ind }}}^{\text {perf }}$ corresponding to $\pi$ via Rozenblyum's additivity theorem.

Remark 3.1.1 It is natural to expect that a solution to the strong quantization problem yields a solution to the weak quantization problem by passing to the category of perfect complexes over the $\mathbb{B D}_{n+1}$-algebra provided by the strong quantization. For this to make sense we need a $\mathbb{B D}$-version of the additivity theorem. In other words we need to know that, for any $k$-linear presentable stable symmetric monoidal $\infty$-category $\mathcal{N}$, there exists a natural equivalence of $\infty$-categories:

$$
\mathbb{B D}_{n+1}-\operatorname{Alg}_{\mathcal{N}} \cong \mathbf{A l g}\left(\mathbb{B D}_{n}-\operatorname{Alg}_{\mathcal{N}}\right)
$$

which specializes to Rozenblyum's additivity at $\hbar=0$ and to Dunn-Lurie's additivity from [Lu4, 5.1.2.2] at $\hbar=1$. Rozenblyum recently proved that the additivity equivalence (3.1) exists and so to any the strong shifted quantization we can indeed associate a weak shifted quantization.

Our main result in this setting is the following unobstructedness theorem

Theorem 3.1.2 ([CPTVV, Theorem 3.5.4]) Let X be a derived Artin stack locally of finite presentation over $k$, equipped with an $n$-shifted Poisson structure $\pi$. If $n>0$ then there is a canonical strong quantization.

The above theorem is analogous to the existence of canonical quantization for unshifted smooth schemes. In fact, at this stage, the proof of the theorem is almost a tautology. Since the operad $\mathbb{E}_{n+1}$ is formal and for $n>0$ its homology 
is $\mathbb{P}_{n+1}$, we can choose a formality equivalence of $k$-dg-operads $\alpha_{n+1}: \mathbb{E}_{n+1} \simeq \mathbb{P}_{n+1}$. The map $\alpha_{n+1}$ induces an equivalence $\mathbb{B D}_{n+1} \simeq \mathbb{P}_{n+1} \otimes_{k} k[\hbar]$ which is the identity mod $\hbar$. Therefore one can consider $\mathcal{P}_{X}(\infty) \otimes_{k} k[\hbar]$ as a stack of $\mathbb{D}_{X_{D R}}(\infty)$-linear graded mixed $\mathbb{B D}_{n+1}$-algebras on $X_{D R}$, and by construction this stack is a strong deformation quantization of $\mathcal{P}_{X}(\infty)$.

By specializing the $\mathbb{B D}_{n+1}$-algebra structure at $\hbar=1$ we can then view $\mathcal{P}_{X}(\infty)$ as a $\mathbb{E}_{n+1}$-algebra in $\mathcal{M}_{\text {Ind }}$. By [Lu4, 5.1.2.2 and 5.1.2.7], the $\infty$-category $\mathcal{P}_{X}(\infty)-\operatorname{Mod}_{\mathcal{M}_{\text {Ind }}}$ has an induced $\mathbb{E}_{n}$-monoidal structure, and one checks that its full sub-category $\mathcal{P}_{X}(\infty)-\operatorname{Mod}_{\mathcal{M}_{\text {Ind }}}^{\text {perf }}$ inherits an $\mathbb{E}_{n}$-monoidal structure that we will denote by $\left(\mathcal{P}_{X}(\infty)-\right.$ $\left.\operatorname{Mod}_{\mathcal{M}_{\text {Ind }}}^{\text {perf }}\right)_{\mathbb{E}_{n}, \pi}$. The subscript $\pi$ indicates that the $\mathbb{E}_{n+1}$-algebra structure on $\mathcal{P}_{X}(\infty)$, hence the induced $\mathbb{E}_{n}$-monoidal structure on $\mathcal{P}_{X}(\infty)-\mathbf{M o d}_{\mathcal{M}_{\text {Ind }}}^{\text {perf }}$, depends on $\pi$, while the subscript $\mathbb{E}_{n}$ records the $\mathbb{E}_{n}$-monoidal structure. This is exactly the deformation of $\operatorname{Perf}(X) \cong \mathcal{P}_{X}(\infty)-\operatorname{Mod}_{\mathcal{M}_{\text {Ind }}}^{\text {perf }}$ that we were looking for. We record this fact in the following

Definition 3.1.3 With the notation above, and $n>0$, the weak quantization of $X$ with infinitesimal $\pi$ is the $\mathbb{E}_{n}$ monoidal $\infty$-category

$$
\operatorname{Perf}(X, \pi):=\left(\mathcal{P}_{X}(\infty)-\operatorname{Mod}_{\mathcal{M}_{I n d}}^{\text {perf }}\right)_{\mathbb{E}_{n}, \pi}
$$

Quantization for $n<0$. The quantization problem for $n$-shifted Poisson structures with $n<0$ can be understood by looking at parameter spaces which are themselves dg schemes. Concretely, let $n<0$ and let $\pi$ be an $n$-shifted Poisson structure on some derived stack $X$ lfp over $k$. Let $\hbar_{2 n}$ a formal variable of cohomological degree $2 n$, and consider the stack $\mathcal{P}_{X}(\infty)\left[\hbar_{2 n}\right]$ of Ind-objects in graded $k(\infty)\left[\hbar_{2 n}\right]$-linear mixed cdgas over $X_{D R}$. Because of the homological shift it is equipped with a $k(\infty)\left[\hbar_{2 n}\right]$-linear $\mathbb{P}_{1-n}$-structure, induced by $\hbar_{2 n} \cdot \pi$ (Theorem 2.3.2). Since $n<0$, this brings us back to the situation of positively shifted Poisson structures.

Proceeding as before, we choose a formality equivalence of $k$-dg-operads $\alpha_{1-n}: \mathbb{E}_{1-n} \simeq \mathbb{P}_{1-n}$, and thus view $\mathcal{P}_{X}(\infty)\left[\hbar_{2 n}\right]$ as a $k(\infty)\left[\hbar_{2 n}\right]$-linear $\mathbb{E}_{1-n}$-algebra. Again by using Dunn-Lurie's additivity [Lu4, 5.1.2.2 and 5.1.2.7], the $\infty$-category $\mathcal{P}_{X}(\infty)-\mathbf{M o d}_{\mathcal{M}_{\text {Ind }}}^{\text {perf }}$ comes equipped with an induced $\mathbb{E}_{-n}$-monoidal structure. We will write $\left(\mathcal{P}_{X}(\infty)-\right.$ $\left.\operatorname{Mod}_{\mathcal{M}_{\text {Ind }}}^{\text {perf }}\right)_{\mathbb{E}_{-n}, \pi}$ for this $\mathbb{E}_{-n}$-monoidal category. Thus for $n<0$ we can define the weak quantization of $X$ with infinitesimal $\pi$ as the $\mathbb{E}_{-n}$-monoidal $\infty$-category

$$
\operatorname{Perf}(X, \pi):=\left(\mathcal{P}_{X}(\infty)-\operatorname{Mod}_{\mathcal{M}_{\text {Ind }}}^{\text {perf }}\right)_{\mathbb{E}_{-n}, \pi}
$$

As before, the underlying $\infty$-category of $\operatorname{Perf}(X, \pi)$ is $\operatorname{Perf}(X) \otimes_{k} k\left[\hbar_{2 n}\right]=: \operatorname{Perf}(X)\left[\hbar_{2 n}\right]$. Hence for $n<0$ our weak quantization of $X$ consists then of the datum of a $\mathbb{E}_{-n}$-monoidal structure on $\operatorname{Perf}(X)\left[\hbar_{2 n}\right]$, and by the strong version of Rozenblyum's additivity, such a quantization can be considered as a deformation of the standard symmetric (i.e. $\left.\mathbb{E}_{\infty^{-}}\right)$monoidal structure on $\operatorname{Perf}(X)\left[\hbar_{2 n}\right]$. Note that this standard symmetric monoidal structure on $\operatorname{Perf}(X)\left[\hbar_{2 n}\right]$ recovers the standard symmetric monoidal structure on $\operatorname{Perf}(X)$ after base change along the canonical map $k\left[\hbar_{2 n}\right] \rightarrow k$.

Remark 3.1.4 This quantization answers a conjecture of Kapustin Kap, 3.2] which concerns the $n=-1$ case. Note that Kapustin considers $\mathbb{Z} / 2$-graded derived categories, and therefore the fact that we work over $k\left[\hbar_{2 n}\right]$ is immaterial: we really obtain a quantization of the $\mathbb{Z} / 2$ perfect derived category of $X$, since $\operatorname{Perf}(X)\left[\hbar_{2 n}\right]$ and $\operatorname{Perf}(X)$ coincide after $\mathbb{Z} / 2$-periodization.

\subsection{Examples and vistas}

(a) Quantization of moduli. The equivalence of shifted symplectic and non-degenerate shifted Poisson structures from Theorem 2.4.2 combined with the $n>0$ (or $n<0$ ) quantization scheme described in the previous section provides a canonical $\mathbb{E}_{n}$-monoidal (or $\mathbb{E}_{-n}$-monoidal) deformation of the $\infty$-category of perfect complexes on the various shifted symplectic moduli stacks listed at the end of section 2.3. For example:

- For a derived Artin stack $X$ locally of finite presentation we obtain a canonical quantization of the shifted cotangent stack $T^{*} X[n]$ for $n \neq 0$. The shifted cotangent stack $T^{*} X[n]$ has a natural $n$-shifted symplectic form Cal2. If we denote the corresponding non-degenerate $n$-shifted Poisson structure by $\pi_{n}$, then the modules over the $\mathbb{E}_{n}$-monoidal (or $\mathbb{E}_{-n}$-monoidal) category $\operatorname{Perf}\left(T^{*} X[n], \pi_{n}\right)$ will be the modules over the $n$-shifted differential operators on $X$. 
- For a complex reductive group $G$ we obtain canonical quantizations of:

- the derived stack $\mathbb{R} \operatorname{Loc}_{G}(M)$ of $G$-local systems on a compact oriented topological manifold $M$ of dimension $\neq 2$;

- the derived stack $\mathbb{R} \operatorname{Loc}_{G}^{D R}(X)$ of algebraic $G$-local systems on a smooth complex projective variety $X$ of dimension $>1$;

- the derived stack $\mathbb{R H i g g s}_{G}(X)$ of algebraic $G$-Higgs bundles on a smooth complex projective variety $X$ of dimension $>1$;

- the derived stack $\mathbb{R} \operatorname{Bun}_{G}(X)$ of algebraic $G$-torsors on a smooth compact Calabi-Yau variety $X$ of dimension $\neq 2$.

Similarly we get quantizations of the stack of perfect complexes on a compact oriented topological manifold $M$ of dimension $\neq 2$, of the derived stack of perfect complexes over $X_{D R}$ for a smooth complex projective variety $X$ of dimension $>1$, of the derived stack of perfect complexes of Higgs bundles on a smooth complex projective variety of dimension $>1$, and on the derived stack of perfect complexes on a smooth compact Calabi-Yau variety of dimension $\neq 2$.

- For a smooth compact Calabi-Yau dg category $T$ of dimension $\neq 2$ we get a canonical weak quantization of the derived moduli stack $\mathcal{M}_{T}$ of compact objects in $T$. For instance we can take $T$ to be the category of graded matrix factorizations of a cubic polynomial $f$ in $3 n$ variables with $n \neq 2$. Applying the general quantization procedure to this setting we get an $(n-2)$-shifted quantization of the moduli stack of graded matrix factorizations of $f$.

(b) Quantization formally at a point. Let $(X, \pi)$ be an $n$-shifted Poisson derived Artin stack locally of finite presentation and let $x: *=\mathbf{S p e c} k \rightarrow X$ be a closed point. It can be checked [CPTVV] Lemma 3.6.1] that any $n$-shifted Poisson structure on $X$ induces an $n$-shifted Poisson structure on the formal completion $\widehat{X}_{x}$ at $x$.

As a (non-mixed) graded cdga over $k, \mathcal{P}_{\widehat{X}_{x}}$ is equivalent to

$$
\operatorname{Sym}\left(\mathbb{L}_{* / \widehat{X}_{x}}[-1]\right) \cong \operatorname{Sym}\left(x^{*} \mathbb{L}_{\widehat{X}_{x}}\right) \cong \operatorname{Sym}\left(x^{*} \mathbb{L}_{X}\right) .
$$

We therefore get a graded mixed $\mathbb{P}_{n+1}$-algebra structure on $\operatorname{Sym}\left(x^{*} \mathbb{L}_{X}\right)$, whose underlying graded mixed cdgas is $\mathcal{P}_{\widehat{X}_{x}}$. After a choice of formality $\alpha_{n+1}$, we get a graded mixed $\mathbb{E}_{n+1}$-structure on $\operatorname{Sym}\left(x^{*} \mathbb{L}_{X}\right)$ whenever $n>0$. When $\pi$ is non-degenerate at $x$ and the induced $n$-shifted Poisson structure on $\mathcal{P}_{\widehat{X}_{x}}$ is strict and constant, then the graded mixed $\mathbb{E}_{n+1}$-structure on $\operatorname{Sym}\left(x^{*} \mathbb{L}_{X}\right)$ can be described explicitly in terms of Kontsevich's graph complex [CPTVV, 3.6.1]. When the underlying dg-Lie algebra is formal, the explicit formula then identifies the $\mathbb{E}_{n+1}$-structure on $\operatorname{Sym}\left(x^{*} \mathbb{L}_{X}\right)$ with the Weyl $n$-algebra recently introduced by Markarian Mar.

(c) Quantization of $B G$. Suppose $G$ is an affine group scheme, and let $X=B G$ be the classifying stack of $G$. Note that $X_{D R}=B\left(G_{D R}\right)$. Let $x: * \rightarrow B G$ be the classifying map of the unit $e: * \rightarrow G$. We have a fiber sequence of groups $\widehat{G}_{e} \longrightarrow G \longrightarrow G_{D R}$, and hence $\widehat{B G}_{x} \simeq B\left(\widehat{G}_{e}\right)$.

As we noted in (b) the pull-back of $\mathcal{P}_{X}$ along $x_{D R}: * \rightarrow B G_{D R}$ is $\mathcal{P}_{\widehat{X}_{x}}$. Thus the symmetric monoidal $\infty$-category

$$
\operatorname{Perf}(B G) \simeq \mathcal{P}_{X}-\operatorname{Mod}_{\epsilon-\mathbf{d g}^{g r}}^{\text {Perf }}
$$

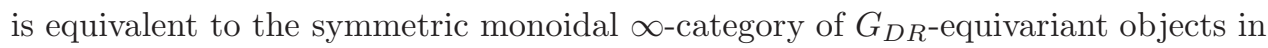

$$
\operatorname{Perf}\left(B \widehat{G}_{e}\right) \simeq \mathcal{P}_{\widehat{X}_{x}}-\operatorname{Mod}_{\epsilon-\operatorname{dg} g r}^{\text {Perf }} .
$$

In view of this the quantization of an $n$-shifted Poisson structure on $B G$ will be determined completely by the $G_{D R^{-}}$ equivariant graded mixed $\mathbb{E}_{n+1}$-algebra structure on $\mathcal{P}_{\widehat{X}_{x}}$ obtained from the equivalence $\alpha_{n+1}: \mathbb{P}_{n+1} \simeq \mathbb{E}_{n+1}$.

This algebra structure can be analyzed in concrete terms. Before we look more closely at the 1 and 2 -shifted cases it is useful to observe that as a graded mixed cdga over $k$ the algebra $\mathcal{P}_{\widehat{X}_{x}} \simeq \mathbb{D}\left(B \widehat{G}_{e}\right)$ admits an explicit description. Indeed, in CPTVV, 3.6.2] it is proven that $\mathbb{D}\left(B \widehat{G}_{e}\right)$ is actually equivalent to the Chevalley-Eilenberg graded mixed cdga $\mathrm{CE}(\mathfrak{g})$ of the Lie algebra $\mathfrak{g}=\operatorname{Lie}(G)$. 
The case $n=1$ for a reductive $G$. For a reductive group $G$ the 1 -shifted Poisson structures on $B G$ are simply elements in $\wedge^{3}(\mathfrak{g})^{G}$. If $\pi$ is such an element, then the induced 1-shifted Poisson structure on the graded mixed cdga $\mathrm{CE}(\mathfrak{g})$ is given explicitly as a semi-strict $\mathbb{P}_{2}$-structure (see $\mathrm{Me}$ ): all structure 2 -shifted polyvectors are trivial except for the 3-ary one which is constant and given by $\pi$.

The weak 1-shifted deformation quantization in particular gives rise to a deformation of the category $\operatorname{Rep}^{f d}(\mathfrak{g})$ of finite dimensional representation of $\mathfrak{g}$ as a monoidal category. For specific choices of $\pi$ we recover familiar monoidal deformations:

Example 3.2.1 Given a non-degenerate invariant pairing $\langle$,$\rangle on \mathfrak{g}$, we can choose $\pi$ as the dual of the $G$-invariant linear form

$$
\wedge^{3} \mathfrak{g} \longrightarrow k, \quad(x, y, z) \longmapsto\langle x,[y, z]\rangle .
$$

Alternatively, any invariant symmetric 2-tensor $t \in \operatorname{Sym}^{2}(\mathfrak{g})^{G}$ leads to such an element $\pi=\left[t^{1,2}, t^{2,3}\right] \in \wedge^{3}(\mathfrak{g})^{G}$. In these cases the deformation of $\operatorname{Rep}^{f d}(\mathfrak{g})$ as a monoidal category can be obtained by means of a deformation of the associativity constraint only (see [Dr1]), which then looks like

$$
\Phi=1^{\otimes 3}+\hbar^{2} \pi+o\left(\hbar^{2}\right) \in U(\mathfrak{g})^{\otimes 3}[[\hbar]] .
$$

The case $n=2$ for a reductive $G$. For a reductive group $G$ the equivalences classes of 2-shifted Poisson structures on $B G$ are in bijection with elements $t \in \operatorname{Sym}^{2}(\mathfrak{g})^{G}$. The induced 2-shifted Poisson structure on the graded mixed cdga $\mathrm{CE}(\mathfrak{g})$ is strict and constant. The graded mixed $\mathbb{E}_{3}$-structure on $\mathrm{CE}(\mathfrak{g})$ given by our deformation quantization then takes the form of a Weyl 3-algebra, as described in Mar.

Note that this graded mixed $\mathbb{E}_{3}$-structure is $G_{D R^{-}}$-equivariant by construction, so it leads to an $\mathbb{E}_{2}$-monoidal deformation of $\operatorname{Perf}(B G)$. This in particular leads to a braided monoidal deformation of $\operatorname{Rep}^{f d}(\mathfrak{g})$.

Such deformation quantizations of $B G$ have already been constructed:

- when $\mathfrak{g}$ is reductive and $t$ is non-degenerate, by means of purely algebraic methods: the quantum group $U_{\hbar}(\mathfrak{g})$ is an explicit deformation of the enveloping algebra $U(\mathfrak{g})$ as a quasi-triangular Hopf algebra.

- without any assumption, by Drinfeld [Dr2, using transcendental methods similar to the ones that are crucial in the proof of the formality of $\mathbb{E}_{2}$.

It is known that Drinfeld's quantization is equivalent to the quantum group one in the semi-simple case (see e.g. [Ka] and references therein).

Remark 3.2.2 It is interesting to note that our quantization, in contrast to Drinfeld's, relies on the formality of $\mathbb{E}_{3}$ rather than on the formality of $\mathbb{E}_{2}$.

(d) Relative and absolute quantization. An important question that is not addressed in this paper or in CPTVV] is the question of quantizing lagrangian structures on maps with shifted symplectic targets or quantizing coisotropic structures on maps with shifted Poisson targets. The quantization problem in this relative stting can be formulated in a manner similar to the absolute quantization from section 3.1

Suppose $(Y, \pi)$ is an $n$-shifted derived Artin stack locally of finite presentation, and $f: X \rightarrow Y$ is a morphism of derived stacks furnished with a coisotropic structure $\kappa$ relative to $\pi$. The $\infty$ categories $\operatorname{Perf}(X)$ and $\operatorname{Perf}(Y)$ are symmetric monoidal categories and via the pullback functor $f^{*}: \operatorname{Perf}(Y) \rightarrow \operatorname{Perf}(X)$ the category $\operatorname{Perf}(X)$ becomes a module over $\operatorname{Perf}(Y)$ so that its $\mathbb{E}_{\infty}$-monoidal structure becomes linear over the $\mathbb{E}_{\infty}$-monoidal structure on $\operatorname{Perf}(Y)$. In other words $f^{*}$ makes $\operatorname{Perf}(X)$ is an $\mathbb{E}_{\infty}$-algebra over $\operatorname{Perf}(Y)$. Assume for simplicity $n>0$. Then the weak quantization of $(Y, \pi)$ gives a deformation of $\operatorname{Perf}(Y)$ to an $\mathbb{E}_{n}$-monoidal category $\operatorname{Perf}(Y, \pi)$. The weak quantization problem for $f$ is to find a concurrent deformation of $\operatorname{Perf}(X)$ as an algebra over $\operatorname{Perf}(Y)$. In other words, we need to deform $\operatorname{Perf}(X)$ to an $\mathbb{E}_{n-1}$-monoidal category $\operatorname{Perf}(X, \kappa)$, so that the functor $f^{*}$ deforms to a functor $\mathbf{q} f^{*}: \operatorname{Perf}(Y, \pi) \rightarrow \operatorname{Perf}(X, \kappa)$ exhibiting $\operatorname{Perf}(X, \kappa)$ as a module, inside $\mathbb{E}_{n-1}$-monoidal $\infty$-categories, over $\operatorname{Perf}(Y, \pi)$, viewed as an $\mathbb{E}_{1}$-algebra in $\mathbb{E}_{n-1}$-monoidal categories. Here again we use Dunn-Lurie additivity [Lu4, §5.1.2] asserting the equivalence between $\mathbb{E}_{1}$-algberas in $\mathbb{E}_{n-1}$-algebras and $\mathbb{E}_{n}$-algebras, in any base symmetric monoidal $\infty$-category.

Note that the extension from the absolute to the relative case is not tautological as the swiss-cheese operad, which governs the deformations of pairs of an $\mathbb{E}_{n}$ algebra and an $\mathbb{E}_{n-1}$ module over it, is not formal. Nevertheless we expect that such relative quantizations are again unobstructed for $n>0$ and we are currently investigating the problem. 
Another interesting problem in this regard is the question of compatibility of quantizations with our standard constructions. A simple instance of this goes as follows. Suppose $Y$ is an $n$-shifted symplectic derived stack (with $n>1$ ) and suppose $f_{1}: X_{1} \rightarrow Y$ and $f_{2}: X_{2} \rightarrow Y$ be two morphisms equipped with lagrangian structures. The derived intersection $Z=X_{1} \times_{Y}^{h} X_{2}$ carries a natural $(n-1)$-shifted symplectic form. We expect that the derived intersection persists in quantizations, i.e. that absolute quantization of $Z$ is the homotopy fiber product of the relative quantizations of $f_{1}$ and $f_{2}$. More precisely, write $\pi$ for the non-degenerate shifted Poisson structure corresponding to the symplectic structure on $Y$ and $\eta$ for the induced $(n-1)$-shifted non-degenerate Poisson structure on $Z$. Let $\kappa_{2} \in \pi_{0} \operatorname{Cois}\left(f_{2}, \pi\right)$ be the non-degenerate coisotropic structure relative to $\pi$ corresponding to the lagrangian structure on $f_{2}$, and let $\kappa_{1} \in \pi_{0} \operatorname{Cois}\left(f_{1},-\pi\right)$ be the non-degenerate coisotropic structure relative to $-\pi$ corresponding to the lagrangian structure on $f_{1}$. Then $\operatorname{Perf}\left(X_{2}, \kappa_{2}\right)$ is an $\mathbb{E}_{n-1}$-algebra over the $\mathbb{E}_{n}$-algebra $\operatorname{Perf}(Y, \pi)$. Similarly $\operatorname{Perf}\left(X_{1}, \kappa_{1}\right)$ is an $\mathbb{E}_{n-1}$-algebra over the $\mathbb{E}_{n}$-algebra $\operatorname{Perf}(Y,-\pi)$ or equivalently $\operatorname{Perf}\left(X_{1}, \kappa_{1}\right)$ is an $\mathbb{E}_{n-1}$-algebra over the opposite of the $\mathbb{E}_{n}$-algebra $\operatorname{Perf}(Y, \pi)$. Conjecturally the quantized $\mathbb{E}_{n-1}$-monoidal category $\operatorname{Perf}(Z, \eta)$ is reconstructed from the $\mathbb{E}_{n-1}$-monoidal category $\operatorname{Perf}\left(X_{1}, \kappa_{1}\right) \otimes_{\operatorname{Perf}(Y, \pi)} \operatorname{Perf}\left(X_{2}, \kappa_{2}\right)$.

(e) Vistas. We conclude our paper by short list of few other directions of investigation, just to stimulate the reader's interest.

First of all we would like to mention the proposal of [Cal, 4.2.2] for a category of lagrangian correspondences $\operatorname{Lagr}_{n}$, based on Thm. 1.2.5 Roughly speaking its objects are $n$-shifted symplectic stacks, and morphisms from $X$ to $Y$ are maps $L \rightarrow X \times Y$ equipped with lagrangian structures (where $X$ is considered with the opposite of the given symplectic structure). Some truncated versions of this category were already considered in Amo-BenB. The details of a complete construction of $\mathbf{L a g r}_{n}$ as an $\infty$-category together with its natural symmetric monoidal structure remain to be written down, but we have no doubts that such a construction exists. This will be an important step toward the study of extended TQFT's (as in [Lu6]) with values in lagrangian correspondences. Building on Remark 2.5.2, one can imagine an analogous construction by replacing shifted symplectic stacks with shifted Poisson stacks, and lagrangian correspondences with coisotropic correspondences. Such a construction is currently being considered by Melani and Safronov, and it might shed some light on Weinstein's original proposal We].

Another promising research program related to the topics treated in this review is the one being pursued, since a few years, by D. Joyce and various collaborators. As part of their project, they use shifted symplectic structures to study Donaldson-Thomas moduli spaces of Calabi-Yau fourfolds, vanishing cycles, and various categorifications of Donaldson-Thomas invariants (see e.g. [BBDJS, Jo-Sa).

The geometry of coisotropic structures for shifted Poisson structures is in a very early stage of development, and a lot of new phenomena need to be properly explored. Just to give one example, the identity map is always endowed with a canonical coisotropic structure, and this produces a map from $n$-shifted Poisson structures to $(n-1)$-shifted Poisson structures, which is worth investigating. If not trivial, this could e.g. connect the $n=2$ to the $n=1$ examples of quantizations of $B G(\mathbf{c})$ above. More generally, having a definition of coisotropic structures that is equivalent to Definition 2.5.1 but avoids reference to the additivity theorem, would be very useful, especially in applications. Some important steps in this direction have been done by P. Safronov [Saf], and more recently by V. Melani and P. Safronov Me-Sa.

Finally, it would be interesting to have a version of Theorem 1.2.1 with target Perf and a stratified topological space as a source. A possible way to include the stratification in our theory is through the use of the MacPherson-Lurie exit paths $\infty$-category [Lu4, A.6]. If this can be accomplished, then a corresponding relative version, as in Remark 1.2.7,

could be relevant for some ideas and conjectures about moduli spaces of constructible sheaves with singular support in a legendrian knot (see [STZ]). A promising preliminary step in this direction is the theory of left and right Calabi-Yau structures on functors developed recently by Toën, and Brav-Dyckerhoff ([To2, p. 228], Br-Dyc).

\section{References}

[AKSZ] M. Alexandrov, M. Kontsevich, A. Schwarz, O. Zaboronsky, The geometry of the master equation and topological quantum field theory, Int. J. Mod. Phys. A, 12 (1997), 1405?1429.

[Amo-BenB] L. Amorim, O. Ben-Bassat, Perversely categorified Lagrangian correspondences, e-Print: arXiv:1601.01536, 56 pages.

[Be-Fa] K. Behrend, B. Fantechi, Symmetric obstruction theories and Hilbert schemes of points on threefolds, Algebra Number Theory 2 (2008), 313?345. 
[Bez-Ka] R. Bezrukavnikov, D. Kaledin, Fedosov quantization in algebraic context. Mosc. Math. J. 4 (2004), no. 3, 559-592.

[BBJ] C. Brav, V. Bussi, D. Joyce, A Darboux theorem for derived schemes with shifted symplectic structure, e-Print: arXiv:1305.6302 (submitted)

[BBDJS] C. Brav, V. Bussi, D. Dupont, D. Joyce, and B. Szendröi, Symmetries and stabilization for sheaves of vanishing cycles, Journal of Singularities 11 (2015), 85-151.

[Br-Dyc] C. Brav, T. Dyckerhoff, Relative Calabi-Yau structures, in preparation.

[Cal] D. Calaque, Lagrangian structures on mapping stacks and semi-classical TFT's, Stacks and Categories in Geometry, Topology, and Algebra, Contemporary Mathematics 643 (2015).

[Cal2] D. Calaque, Deformed shifted cotangent stacks in derived Poisson geometry, preprint, 2016,9 pages.

[CPTVV] D. Calaque, T. Pantev, B. Toën, M. Vaquié, G. Vezzosi, Shifted Poisson Structures and Deformation Quantization, e-Print: arXiv:1506.03699v3 (submitted).

[Coh] F. Cohen in F. Cohen, T. J. Lada, and J. P. May, The homology of iterated loop spaces, Springer-Verlag, Berlin, 1976.

[Co-Gwi] K. Costello, O. Gwilliam, Factorization algebras in quantum field theory Volume II, Book draft, December 2015, available at http://people.mpim-bonn.mpg.de/gwilliam/

[Dr1] V. Drinfeld, Quasi-Hopf algebras, Leningr. Math. J. 1 (1990), no. 6, 1419-1457.

[Dr2] V. Drinfeld, On quasi-triangular quasi-Hopf algebras and a group closely related with Gal( $\overline{\mathbb{Q}}, \mathbb{Q})$, Leningr. Math. J. 2 (1991), no. 4, 829-860.

[Gi-Ba] V. Ginzburg, V. Baranovsky, Gerstenhaber-Batalin-Vilkoviski structures on coisotropic intersections, to appear in Math. Res. Lett.

[Hen] B. Hennion, Tangent Lie algebra of derived Artin stacks ., e-Print: arXiv:1312.3167, to appear in Journal für die reine und angewandte Mathematik (Crelles Journal).

[Hir] P. S. Hirschhorn, Model categories and their localizations, Mathematical Surveys and Monographs (99), AMS, 2003.

[HS] A. Hirschowitz, C. Simpson. Descente pour les n-champs, e-Print: arXiv math/9807049.

[Hov] M. Hovey, Model categories, Mathematical Surveys and Monographs (63), AMS, 2007.

[Hoy] M. Hoyois, The fixed points of the circle action on Hochschild homology, e-Print: arXiv:1506.07123.

[Jo-Sa] D. Joyce and P. Safronov, Lagrangian neighborhood theorem for shifted symplectic derived schemes, e-Print: arXiv:1509.08081

[JS] D. Joyce and Y. Song, A theory of generalized Donaldson-Thomas invariants, Mem. Amer. Math. Soc. 217 (2012), no. 1020 .

[Kap] A. Kapustin, Topological Field Theory, Higher Categories, and Their Applications, Proceedings of the International Congress of Mathematicians Hyderabad, India, 2010.

[Ka] C. Kassel, Quantum groups, Graduate Texts in Mathematics 155, 1995, Springer.

[Ko1] M. Kontsevich, Deformation quantization of Poisson manifolds, Lett. Math. Phys. 66 (2003), $157-216$.

[KS] M. Kontsevich and Y. Soibelman, Stability structures, motivic Donaldson-Thomas invariants and cluster transformations, e-Print: arXiv:0811.2435, 2008.

[Ill] L. Illusie, Complexe cotangent et déformations II, Lecture Notes in Mathematics 283, Springer Verlag, Berlin, 1972. 
[Lu1] J. Lurie, Higher Topos Theory, Annals of Mathematics Studies, 170, Princeton University Press, Princeton, NJ, 2009. xviii $+925 \mathrm{pp}$.

[Lu2] J. Lurie, Formal Moduli Problems, Vol. 2, Proceedings of the International Congress of Mathematicians 2010 (R. Bhatia, A. Pal, G. Rangarajan, V. Srinivas, M. Vanninathan editors), World Scientific.

[Lu3] J. Lurie, DAG series, V to XIV, available at http://www.math.harvard.edu/ lurie/

[Lu4] J. Lurie, Higher Algebra, September 2014, available at http://www.math.harvard.edu/ lurie/.

[Lu5] J. Lurie, Spectral algebraic Geometry, draft February 2016, available at http://www.math.harvard.edu/ lurie/.

[Lu6] J. Lurie, On the Classification of Topological Field Theories, Current Developments in Mathematics Volume 2008 (2009), 129-280.

[Mar] N. Markarian, Weyl n-algebras, e-Print: arXiv:1504.01931, 2015.

[Me] V. Melani, Poisson bivectors and Poisson brackets on affine derived stacks, to appear in Advances in Mathematics.

[Me-Sa] V. Melani, P. Safronov, Shifted Poisson stuctures and coisotropic intersections, in preparation.

[Ni-Sa] Niklaus, S. Sagave, Presentably symmetric monoidal infinity-categories are represented by symmetric monoidal model categories, e-Print: arXiv:1506.01475.

[PTVV] T. Pantev, B. Toën, M. Vaquié, G. Vezzosi, Shifted symplectic structures, Publ. Math. IHES, June 2013, Volume 117, Issue 1, pp 271-328.

[Saf] P. Safronov, Poisson reduction as a coisotropic intersection, e-Print: arXiv:1509.08081,

[Sch] M. Schlessinger, Functors of Artin rings, Transactions of the American Mathematical Society 130: 208-222 (1968).

[STZ] V. Shende, D. Treumann, E. Zaslow, Legendrian knots and constructible sheaves, e-Print: arXiv:1402.0490.

[Spa] T. Spaide, Shifted symplectic structures on spaces of framed maps, thesis, University of Pennsylvania, 2015.

[To1] B. Toën, Higher and derived stacks: a global overview. Algebraic geometry - Seattle 2005. Part 1, 435-487, Proc. Sympos. Pure Math., 80, Part 1, Amer. Math. Soc., Providence, RI, 2009.

[To2] B. Toën, Derived Algebraic Geometry, EMS Surv. Math. Sci. 1 (2014), no. 2, 153-245.

[To-Va] B. Toën, M. Vaquié, Moduli of objects in dg-categories, Ann. Sci. de l'ENS Volume 40 (2007) Issue 3, Pages 387-444.

[HAG-II] B. Toën, G. Vezzosi, Homotopical algebraic geometry II: Geometric stacks and applications, Mem. Amer. Math. Soc. 193 (2008), no. 902, x+224 pp.

[To-Ve-1] B. Toën, G. Vezzosi, Caractères de Chern, traces équivariantes et géométrie algébrique dérivée, Selecta Mathematica 21 (2015), no. 2, 449-554.

[To-Ve-2] B. Toën, G. Vezzosi, Algèbres simpliciales $S^{1}$-équivariantes, théorie de de Rham et théorèmes HKR multiplicatifs, Compositio Math., 147 / Issue 06, n. 11, pp. 1979-2000 (2011).

[Ty] A. N. Tyurin, Fano versus Calabi-Yau, The Fano Conference, Univ. Torino, Turin, 2004, pp. 701-734.

[Vez1] G. Vezzosi, Derived critical loci I - Basics, e-Print: arXiv:1109.5213.

[Vez2] G. Vezzosi, Quadratic forms and Clifford algebras on derived stacks, e-Print: arXiv:1309.1879 (submitted).

[Vez3] G. Vezzosi, A model structure on relative dg-Lie algebroids, Proceedings of CATS4: Stacks and Categories on Geometry, Topology, and Algebra, Contemporary Mathematics 643, 111-118.

[We] A. Weinstein, Coisotropic calculus and Poisson groupoids, J. Math. Soc. Japan 40 (1988), 705-727.

[Ye] A. Yekutieli, Twisted deformation quantization of algebraic varieties. Adv. Math. 268 (2015), 241-305. 
Tony Pantev, University of Pennsylvania, tpantev@math.upenn.edu Gabriele Vezzosi, Università Di Firenze, gabriele.vezzosi@unifi.it 